J. Dorfmeister

Nagoya Math. J.

Vol. 114 (1989), 77-122

\title{
PROOF OF THE RADICAL CONJECTURE FOR HOMOGENEOUS KAHLER MANIFOLDS
}

\author{
JOSEF DORFMEISTER
}

\section{Introduction}

In 1967 Gindikin and Vinberg stated the Fundamental Conjecture for homogeneous Kähler manifolds. It (roughly) states that every homogeneous Kähler manifold is a fiber space over a bounded homogeneous domain for which the fibers are a product of a flat with a simply connected compact homogeneous Kähler manifold. This conjecture has been proven in a number of cases (see [6] for a recent survey). In particular, it holds if the homogeneous Kähler manifold admits a reductive or an arbitrary solvable transitive group of automorphisms [5]. It is thus tempting to think about the general case. It is natural to expect that lack of knowledge about the radical of a transitive group $G$ of automorphisms of a homogeneous Kähler manifold $M$ is the main obstruction to a proof of the Fundamental Conjecture for $M$. Thus it is of importance to consider the Kähler algebra generated by the radical of the Lie algebra of $G$. Computations in this context suggest that one rather considers Kähler algebras generated by an arbitrary solvable ideal. In this context the Radical Conjecture for Kähler algebras was formulated [6]: Assume that the Kähler algebra $(\mathfrak{g}, \mathfrak{f}, j, \rho$ ) is generated by a solvable ideal $\mathfrak{r}$ of $\mathfrak{g}$, i.e. $\mathfrak{g}=\mathfrak{r}+j \mathfrak{r}+\mathfrak{f}$, then $\mathfrak{g}=\mathfrak{g}+\mathfrak{l}$, where $\mathfrak{g} \cap \mathfrak{l}=0, j \mathfrak{g} \subset \mathfrak{g}$ (after an inessential change of $j$ ), and $\mathfrak{g}$ is a solvable Kähler algebra.

If $\mathfrak{r}$ is abelian, a direct proof of the Radical Conjecture can be given (following closely a proof of Gindikin, Piatetskii-Shapiro and Vinberg [8]. A proof of the Radical Conjecture proceeds by induction on $\operatorname{dim} x$. It was started in [6]. Since the case that $\mathfrak{x}$ is abelian was already settled one considers a maximal ideal $\mathfrak{n}$ of $\mathfrak{g}$ properly contained in $\mathfrak{r}$ and sets $\mathfrak{g}^{\prime}=\mathfrak{n}$ $+j \mathfrak{l}+$. For this Kähler algebra the Radical Conjecture already holds. Generalizing constructions of Gindikin, Piatetskii-Shapiro and Vinberg it

Received July 6, 1987. 
was shown in [6] that only three cases have to be considered (one is that $n+j n$ is essentially an abelian Kähler algebra, the other two are characterized by the distributions of eigenvalues of a maximal idempotent in $\mathfrak{g}^{\prime}$, where $\mathfrak{g}^{\prime}$ is associated with $\mathfrak{g}^{\prime}$ via the Radical Conjecture, $\mathfrak{g}^{\prime}=\mathfrak{g}^{\prime}+\mathfrak{f}$ ).

In the present paper, we continue and finish the proof of the Radical Conjecture.

The details are rather technical and involved. We thus only want to point out that in Case 1 (where $\mathfrak{n}+j \mathfrak{n}$ is essentially abelian) we prove a statement which is stronger than the Radical Conjecture. In Case 3 we combine the description of the representations of $\operatorname{sl}(2, Z)$ with results on the Kantor-Koecher-Tits construction of Lie algebras with more standard techniques of Kähler algebras to prove the Radical Conjecture.

Finally, we would like to note that the Radical Conjecture and a substantial part of its proof have been used in the recent proof of the Fundamental Conjecture (jointly with K. Nakajima).

I would like to thank $K$. Johnson for making me aware of [16] and E. Neher for helpful discussions and information about Jordan triples (the structure of which is used in case 3).

I would like to express great appreciation to K. Nakajima for his careful reading of a preliminary version of this paper, in particular for many critical and helpful remarks and for simplifying Lemma 1.5, Lemma 2.17 and section 3.7. He also pointed out to me a subcase of case 3 which was not discucssed originally.

\section{$\S 1$. Case 1: The Lie algebra $n+j n$ is the modification of an abelian Kähler algebra}

1.1. As in [6;4.33] we consider a Kähler algbra $(\mathfrak{g}, \mathfrak{f}, j, \rho)$ and a solvable ideal $\mathfrak{x}$ of $\mathfrak{g}$ satisfying $\mathfrak{g}=\mathfrak{x}+j \mathfrak{x}+\mathfrak{f}$. We assume that the Radical Conjecture holds if $\operatorname{dim} \mathfrak{r} \leq N-1$. We assume $\operatorname{dim} \mathfrak{r}=N$ and we may assume $\mathfrak{r} \subset \operatorname{nil}(\mathfrak{g})$. Moreover we can assume that the dimension of $\mathfrak{r}$ is minimal among those solvable ideals $\mathfrak{u}$ of $\mathfrak{g}$ for which $\mathfrak{g}=\mathfrak{u}+j \mathfrak{u}+\mathfrak{f}$ holds. We choose an ideal $\mathfrak{n}$ of $\mathfrak{g}$ which satisfies $\mathfrak{r} \supseteq \mathfrak{n} \supset[\mathfrak{r}, \mathfrak{r}]$ and is maximal with this property. Because the case of an abelian $x$ has been settled in [6] we can also assume $\mathfrak{n} \neq 0$.

We set $\mathfrak{g}^{\prime}=\mathfrak{n}+j \mathfrak{n}+\mathfrak{l}$ and apply the Radical Conjecture to $\mathfrak{g}^{\prime}$. Hence $\mathfrak{g}^{\prime}=\mathfrak{a}+t+\mathfrak{f}$ where $\mathfrak{a}+t$ is a solvable Kähler algebra. The case under consideration in this paper is defined by $\mathfrak{t}=0$, i.e. $\mathfrak{g}^{\prime}=\mathfrak{a}+\mathfrak{f}$ where $\mathfrak{a}$ is the 
modification of an abelian Kähler algebra. Such algebras have been investigated in [5].

1.2. From [5;3.3] we know $\mathfrak{a}=\mathfrak{n}+j \mathfrak{n}=\hat{\mathfrak{a}}_{0}+\hat{\mathfrak{a}}_{1}$ where $\hat{\mathfrak{a}}_{0}$ is an abelian ideal of $\mathfrak{a}$ and $\hat{a}_{1}$ is an abelian subalgebra. Moreover $\hat{a}_{0}=[\mathfrak{a}, \mathfrak{a}]$. Because $\mathfrak{n}$ acts nilpotently on $\mathfrak{g}$ we have $[\mathfrak{n}, \mathfrak{n}]=0$. Set $\mathfrak{n}_{0}=\hat{\mathfrak{a}}_{0} \cap \mathfrak{n}$ and $\mathfrak{n}_{1}=\hat{\mathfrak{a}}_{1} \cap \mathfrak{n}$.

LEMMA. $\mathfrak{n}=\mathfrak{n}_{0}+\mathfrak{n}_{1}$.

Proof. Let $n \in \mathfrak{n}$ and $n=a_{0}+a_{1}, a_{j} \in \hat{\mathfrak{a}}_{j}$. From the fact that $\mathfrak{a}$ is the modification of an abelian Kähler algebra we get $[b, n]=D(b) n-D(n) b$ for all $b \in \mathfrak{a}$. As $a d n$ is nilpotent we have $D(n)=0$. Further, from [5:3.3] we know $D(b) \hat{a}_{1}=0$. Therefore $[b, n]=D(b) \mathfrak{a}_{0} \in \mathfrak{n}$ for all $b \in \mathfrak{a}$. Using $\hat{a}_{0}$ $=[\mathfrak{a}, \mathfrak{a}]$ we derive from this $a_{0} \in \mathfrak{n}$. The lemma follows.

1.3. We note that $\mathfrak{n}_{0}+j \mathfrak{n}_{0}$ is contained in the commutator $\hat{a}_{0}=[\mathfrak{a}, \mathfrak{a}]$ of the solvable Lie algebra $a$. Hence $\operatorname{ad}\left(\mathfrak{n}_{0}+j \mathfrak{n}_{0}\right)$ is a commuting family of nilpotent derivations of $\mathrm{g}$. In particular we have $\left[\mathfrak{n}_{0}+j \mathfrak{n}_{0}, \mathfrak{n}\right]=0$.

In contrast to this the family $\operatorname{ad}\left(\mathfrak{n}_{1}+j \mathfrak{n}_{1}\right)$ is abelian but does not consist in general of nilpotent derivations.

1.4. Consider $\mathfrak{g}^{\prime}=\mathfrak{a}+\mathfrak{f}$. From the Radical Conjecture we know $\mathfrak{a} \cap \mathfrak{f}$ $=0$ and $\mathfrak{a}$ is a solvable Kähler subalgebra of $\mathfrak{g}^{\prime}$. Let $\mathfrak{f}_{0} \subset \mathfrak{f}$ be an ideal of $\mathrm{g}^{\prime}$. We know that $\mathfrak{i}$ is the Lie algebra of a compact group and $\mathfrak{f}_{0}$ is an ideal of $\mathfrak{f}$. Hence $\mathfrak{f}=\left(z_{1}+z_{0}\right) \oplus \mathfrak{I}_{1}^{\prime} \oplus \mathfrak{l}_{0}^{\prime}$ where $\mathfrak{l}_{0}=\mathfrak{f}_{0}^{\prime} \oplus \mathfrak{z}_{0}, \mathfrak{f}_{1}^{\prime}, \mathfrak{I}_{0}^{\prime}$ semisimple, $z_{0} z_{1}$ abelian. Let $\mathfrak{h}$ be a maximal semisimple subalgebra of $\mathfrak{g}^{\prime}$ containing $\mathfrak{f}_{1}^{\prime} \oplus \mathfrak{F}_{0}^{\prime}$. Then $\mathfrak{f}_{0}^{\prime}$ is an ideal of $\mathfrak{h}$ and we get $\mathfrak{h}=\mathfrak{h}_{1}^{\prime} \oplus \mathfrak{f}_{0}^{\prime}$. Moreover, $\left[\operatorname{rad} \mathfrak{g}^{\prime}\right.$ $\left.+\mathfrak{h}_{1}^{\prime}, \mathfrak{f}_{0}^{\prime}\right]=0$. Since $\mathfrak{n} \subset \operatorname{rad} \mathfrak{g}^{\prime}$ we can assume w.r.g. that $j \mathfrak{n}$ projects trivially onto $\mathfrak{f}_{0}^{\prime}$. Hence $\left[\mathfrak{a}, \mathfrak{f}_{0}^{\prime}\right]=0$ and $\mathfrak{g}^{\prime}=\left(\mathfrak{a}+z_{1}+z_{0}+\mathfrak{f}_{1}^{\prime}\right) \oplus \mathfrak{f}_{0}^{\prime}$ is a direct sum of Lie agebras. Moreover, $z_{0}$ is an ideal of $\mathrm{g}^{\prime}$ and $z_{0} \subset \mathrm{Cad} \mathrm{g}^{\prime}$. But $z_{0} \cap$ $\operatorname{nil}\left(\mathfrak{g}^{\prime}\right)=0$ since nil $\left(\mathfrak{g}^{\prime}\right)$ operates nilpotently on $g$ whereas $\mathfrak{f}_{0}$ acts semisimply. From this we conclude $\left[\mathfrak{g}^{\prime}, \partial_{0}\right]=0$.

Therefore, when considering $\mathfrak{g}^{\prime}$ only, we may assume that $\mathfrak{k}$ does not contain any ideal of $\mathfrak{g}^{\prime}$. But then we have a faithful representation $\psi$ of $\mathfrak{g}^{\prime}$ as affine transformations of the complex vector space $\mathfrak{a}$. The elements of $\mathfrak{f}$ act linearly on $\mathfrak{a}$ and the elements of $\mathfrak{a}$ by $z \mapsto a+D(a) z, z \in \mathfrak{a}, a \in \mathfrak{a}$ (see $[5 ; 3.5])$. Considering the linear parts of these transformations we see that $D(\mathfrak{a})+\psi(\mathfrak{f})=\mathbb{B}$ is a Lie algebra of skewadjoint endomorphisms of $\mathfrak{a}$; moreover, $D(\mathfrak{a})$ is an abelian subalgebra, $\psi(\mathfrak{l})$ is a subalgebra and (possibly changing $j$ inessentially) we also can assume $D(\mathfrak{a}) \cap \psi(\mathfrak{f})=0$. We 
split $\mathbb{B}=\oplus \mathbb{B S}_{i}+3$ into simple summands $\mathbb{S}_{i}$ and its center 3. Applying [16: Theorem 1.1] to the projections $D_{i}(\mathfrak{a})$ and $\psi_{i}(\mathfrak{l})$ of $D(\mathfrak{a})$ and $\psi(\mathfrak{l})$ onto $\mathbb{G S}_{i}$ shows that $D_{i}(\mathfrak{a}) \subset \psi_{i}(\mathfrak{l})$ holds. Therefore $D(a) \subset \psi(\mathfrak{f}) \bmod$ center $(D(\mathfrak{a})$ $+\psi(\mathfrak{f}))$. This implies:

Lemma. After an inessential change of $j$ we can assume $[D(\mathfrak{a}), \psi(\mathfrak{f})]$ $=0$.

For the original Lie Algebra $\mathfrak{g}^{\prime}$ (ideal in $\mathfrak{f}^{\prime}$ permitted) this implies (after some inessential change of $j$ ):

Corollary 1 . a) $[\mathfrak{f}, \mathfrak{a}] \subset \mathfrak{a}$,

b) $D([\mathfrak{f}, \mathfrak{a}])=0$,

c) $[D(\mathfrak{a}), \operatorname{ad} \mathfrak{H} \mid \mathfrak{a}]=0$,

d) $[\operatorname{ad} \mathfrak{f} \mid a, j]=0$.

Proof. Write $\mathfrak{f}=\mathfrak{z}+\mathfrak{f}_{1}^{\prime}+\mathfrak{f}_{0}^{\prime}$ and represent $\mathfrak{a}$ by affine transformations on some complex vector space $(\cong a)$. Then $[k, a]=[(0, \psi(k)),(a, D(a))]=$ $(\psi(k) a,[\psi(k), D(a)])=(\psi(k) a, 0)=(\psi(k) a, D(\psi(k) a))-(0, D(\psi(k) a))$ where the last summand has to be in $\mathfrak{f}$. By our assumption, we may assume $a=j n$ for some $n \in \mathfrak{n}$, whence $\psi(k) a=i(\psi(k) n)$. Changing $j$ on $\psi(k) \mathfrak{n}$ inessentially we obtain $D(i(\psi(k) \mathfrak{n}))=0$, proving a) and b). Part c) has been known before and d) follows from a).

We retain the notation of 1.2 and 1.3 and write $\hat{a}_{1}=\hat{a}_{10}+\hat{a}_{11}$ as in [5:3.3].

Corollary 2. a) $\left[\mathfrak{f}, \hat{a}_{i 0}\right] \subset \hat{a}_{10}$,

b) $\left[\mathfrak{f}, \hat{a}_{11}\right]=0$,

c) $\left[\mathfrak{f}, \hat{a}_{0}\right] \subset \hat{a}_{0}$.

Proof. Since $[\mathfrak{f}, \mathfrak{a}] \subset \mathfrak{a}$ and $\hat{a}_{0}=[\mathfrak{a}, \mathfrak{a}]$ part $\mathrm{c}$ ) is clear. We know that $\mathfrak{f}$ acts skewadjoint on $\mathfrak{a}$, whence $\left[\mathfrak{f}, a_{1}\right] \subset a_{1}$. By Corollary 1 we have $\left[\mathfrak{f}, \mathfrak{a}_{1}\right] \subset \hat{a}_{10}$ and a) and b) follow.

1.5. Let $n \in \mathfrak{n}_{1}$ and write ad $j n=D+N$ where $D$ is the semisimple part of $\operatorname{adjn}$ and $N$ its nilpotent part.

The proof of the following lemma is a simplified version of the author's criginal proof. We follow a suggestion of K. Nakajima.

Lemma. For $n \in \mathfrak{n}_{1}$, the semisimple part $D$ of ad $j n$ has only imaginary eigenvalues. 
Proof. Let $R$ be the real part of $D$ and $\mathfrak{g}=\oplus \mathfrak{g}_{\lambda}$ be the decomposition of $\mathfrak{g}$ into eigenspaces for $R$. Because $D \mid \mathfrak{g}^{\prime}$ has only imaginary eigenvalues we get

(1) $\mathfrak{g}^{\prime} \subset \mathfrak{g}_{0}$.

The integrability condition of $\mathfrak{g}$ implies $\left[j n, j x_{\lambda}\right]=j\left[j n, x_{\lambda}\right] \bmod \mathfrak{g}^{\prime}$ for all $x_{\lambda} \in g_{\lambda}$. Therefore

(2) $j \mathfrak{g}_{\lambda} \subset \mathfrak{g}_{\lambda}+\mathfrak{g}^{\prime}$.

Moreover, by an inessential change of $j$ we may even assume

(3) $j \mathfrak{g}_{\lambda} \subset \mathfrak{g}_{\lambda}+\mathfrak{n}+j \mathfrak{n}$.

Let $\lambda$ be the eigenvalue of $R$ with maximal absolute value. We may assume $\lambda \geq 0$. Suppose $\lambda>0$. Then $\left[g_{\lambda}, g_{2}\right]=0$. Therefore

(4) $\mathfrak{g}_{\lambda}+\mathfrak{n}+j \mathfrak{n}$ is a solvable Kähler algebra.

By [5] we know that ad $j n$ has only purely imaginary eigenvalues in this algebra. This is a contradiction; hence we obtain $\lambda=0$, finishing the proof of the lemma.

1.6. In the last section we have seen that the semisimple parts of elements of $\mathfrak{n}_{1}$ have only imaginary eigenvalues. In the following sections we show that, by a change of $\rho$ and a modification, we can remove these semisimple parts entirely.

We consider the eigenspace decomposition of $g$ relative to $-D^{2}, D$ the semisimple part of ad $j n, n \in \mathfrak{n}_{1}$. Then there exist subspaces $\mathfrak{g}_{\alpha}, \alpha \geq 0$, and an endomorphism $I$ of $g$ such that

1) $I^{2}=-\mathrm{id}$,

2) $D \mid g_{\alpha}=\alpha I$,

3) $\mathfrak{g}=\bigoplus_{\alpha \geq 0} \mathfrak{g}_{\alpha}$.

From Corollary 1, a) of 1.4 we know that $\mathfrak{a}$ is an ideal of $\mathfrak{g}^{\prime}=\mathfrak{f}+\mathfrak{a}$. Since $a$ is solvable we have $a \subset \operatorname{rad} \mathfrak{g}^{\prime}$. Therefore $[\mathfrak{f}, \mathfrak{a}] \subset\left[\mathfrak{g}^{\prime}, \mathfrak{g}^{\prime}\right] \cap \operatorname{rad} \mathfrak{g}^{\prime}$ $=\operatorname{nil}\left(\mathfrak{g}^{\prime}\right)$. In particular ad $[\mathfrak{f}, \mathfrak{a}]$ is nilpotent on $\mathfrak{g}$. Moreover, from Corollary 1. d) we derive $j\left[\mathfrak{f}, \mathfrak{n}_{1}\right]=\left[\mathfrak{f}, j \mathfrak{n}_{1}\right] \subset[\mathfrak{f}, \mathfrak{a}]$. Hence ad $j\left[\mathfrak{f}, \mathfrak{n}_{1}\right]$ is nilpotent. Hence we can (and will) assume that $n$ is taken from some $\mathfrak{l}$-invariant complement of $\left[\mathfrak{f}, \mathfrak{n}_{1}\right]$ in $\mathfrak{n}_{1}$. But then $[\mathfrak{f}, n]=0$.

1.7. In this section we want to prove that (after an inessential change 
of $j$ ) we have

Lemma. a) $j \mathfrak{g}_{\alpha} \subset \mathfrak{g}_{\alpha}$ for all $\alpha$.

b) $D j x_{\alpha}=j D x_{\alpha}$ for all $\alpha$ and all $x_{\alpha} \in \mathfrak{g}_{\alpha}$.

Proof. From the integrability condition we get

$$
\left[j n, j x_{\alpha}\right]=j\left[j n, x_{\alpha}\right] \bmod \mathfrak{g}^{\prime} \text { for all } x_{\alpha} \in \mathfrak{g}_{\alpha} .
$$

Because ad $j n$ leaves $g^{\prime}$ invariant, we also have

$$
D j x_{\alpha}=j D x_{\alpha} \bmod g^{\prime} \text {. }
$$

This implies

$$
j \mathfrak{g}_{\alpha} \subset \mathfrak{g}_{\alpha}+\mathfrak{g}^{\prime} .
$$

Hence, for $x_{\alpha} \in g_{\alpha}$ we have $j x_{\alpha}=y_{\alpha}+g_{0}^{\prime}+\sum_{\beta \neq 0} g_{\beta}^{\prime}$ where $g_{\lambda}^{\prime} \in \mathfrak{g}_{\lambda}^{\prime}$ and - w.r.g. $-g_{0}^{\prime} \in \mathfrak{a}$. We want to use this expansion of $j x_{\alpha}$ in the integrability condition $\left[j n, j x_{\alpha}\right]=j\left[j n, x_{\alpha}\right]+j\left[n, j x_{\alpha}\right]+\left[n, x_{\alpha}\right]+k$. Therefore, we have to study $\left[n, j x_{\alpha}\right]$. It is easy to see that $\mathfrak{g}_{\beta}^{\prime} \subset \hat{a}_{0}$ for all $\beta \neq 0$. Hence $\left[n, \mathfrak{g}_{\beta}^{\prime}\right]$ $=0$ for all $\beta \neq 0$. From the description of $a$ we derive immediately $\mathfrak{g}_{0}^{\prime}=\hat{\mathfrak{a}}_{1}+\left(\mathfrak{g}_{0}^{\prime} \cap \hat{\mathfrak{a}}_{0}\right)+\mathfrak{f}$.

But $n \in \hat{a}_{1}$, whence $\left[n, \hat{a}_{1}\right]=0$. Moreover, $n \in \operatorname{nil}(\mathfrak{g})$ implies $\left[n, \hat{a}_{0}\right]=0$. We have thus shown $\left[n_{1}, \mathfrak{a}\right]=0$, and in particular

$$
\left[n, j x_{\alpha}\right] \in \mathfrak{g}_{\alpha}^{\prime} \quad \text { for all } x_{\alpha} \in \mathfrak{g}_{\alpha} .
$$

But $g_{\lambda}^{\prime}$ is $j$-invariant for all $\lambda$, whence

$$
\left[j n, j x_{\alpha}\right]-j\left[j n, x_{\alpha}\right] \in \mathfrak{g}_{\alpha}^{\prime}+\mathfrak{l} .
$$

From Corollar 1, d) of 1.4 and our choice of $n$ we know $[j n, \mathfrak{t}]=j[n, \mathfrak{l}]$ $=0$. In particular, $D \mathfrak{f}=0$. Because $\left[j n, \mathfrak{g}_{\alpha}^{\prime}\right] \subset \mathrm{g}_{\alpha}^{\prime}$ we derive from (5)

$$
D j x_{\alpha}=j D x_{\alpha}+g_{\alpha}^{\prime \prime}+k^{\prime \prime} \quad \text { for some } g_{\alpha}^{\prime \prime} \in g_{\alpha}^{\prime}, k^{\prime \prime} \in \mathfrak{I} .
$$

Applying $D$ to (6) we get $D^{2} j x_{\alpha}=-\alpha^{2} j x_{\alpha}+g_{\alpha}^{\prime \prime \prime}+k^{\prime \prime \prime}$. We compare the $\mu$-components and see $-\mu^{2}\left(j x_{\alpha}\right)_{\mu}=-\alpha^{2}\left(j x_{\alpha}\right)_{\mu}$ for $\mu \neq \alpha, 0$. This implies $j \mathfrak{g}_{\alpha} \subset \mathfrak{g}_{\alpha}+g_{0}$ and in particular, $j \mathfrak{g}_{0} \subset \mathfrak{g}_{0}$. Let $\alpha \neq 0$. Comparing for $\mu=\alpha$ and $\mu=0$ gives $\mathfrak{g}_{\alpha}^{\prime \prime \prime}=0$ and $\left(j x_{\alpha}\right)_{0} \in \mathfrak{f}$. This implies $j \mathfrak{g}_{\alpha} \subset \mathfrak{g}_{\alpha}+\mathfrak{f}$. After an inessential change of $j$ we can assume (proving a)).

$$
j \mathfrak{g}_{\alpha} \subset \mathfrak{g}_{\alpha} \quad \text { for all } \alpha .
$$

It is easy to verify that for every $\alpha, \mathfrak{g}(\alpha)=\bigoplus_{n \in Z} \mathfrak{g}_{u_{\alpha}}$ is a $j$-invariant 
subalgebra of $\mathfrak{g}$. Moreover, $\mathfrak{g}_{\lambda}=\mathfrak{r}_{2}+j \mathfrak{r}_{2}$ for $\lambda \neq 0$ and $\mathfrak{g}_{0}=\mathfrak{r}_{0}+j \mathfrak{r}_{0}+\mathfrak{f}$. Therefore we can apply the induction hypothesis to $g(\alpha)$ when $\mathfrak{g}(\alpha) \neq \mathfrak{g}$. In case $\mathfrak{g}(\alpha) \neq \mathfrak{g}$ for all $\alpha$, the lemma follows. Assume now $g(\alpha)=\mathfrak{g}$. We can assume $\alpha=1$. Applying the induction hypothesis to $g(m), m$ $=2,3, \cdots$ we see that the lemma holds for all $m \neq 1$. We write ad $j n$ $=D+N$, where $N$ is the nilpotent part of ad $j n$, and use the induction hypothesis for $\mathfrak{g}(2)$ to see for $x_{1}, y_{1} \in \mathfrak{g}_{1}:(d / d t) \rho\left(e^{\operatorname{tad} j n} x_{1}, e^{\operatorname{tad} j n} y_{1}\right)=\rho\left(j n, e^{\operatorname{tad} j n}\right.$ $\left.\times\left[x_{1}, y_{1}\right]\right)=\rho\left(j n, e^{t D} e^{t N}\left[x_{1}, y_{1}\right]\right)=\rho\left(j n, e^{t N}\left[x_{1}, y_{1}\right]\right)$. Here the last term is a polynomial in $t$. An integration yields

$$
\rho\left(e^{\mathrm{tad} j n} x_{1}, e^{\mathrm{tad} j n} y_{1}\right)=\rho\left(x_{1}, y_{1}\right)+c_{1} t+c_{2} t^{2}+\cdots .
$$

We have $e^{\operatorname{tad} j n}=e^{t N} e^{t D}$ where $e^{t D} x$ is bounded for all $x \in \mathfrak{g}$ and $e^{t N} y$ is a polynomial in $t$. Comparing like powers of $t$ on both sides gives

$$
\rho\left(e^{t D} x_{1}, e^{t D} y_{1}\right)=\rho\left(x_{1}, y_{1}\right) \quad \text { for all } x_{1}, y_{1} \in \mathfrak{g}_{1} \text {. }
$$

In particular, we obtain $\rho\left(D x_{1}, y_{1}\right)+\rho\left(x_{1}, D y_{1}\right)=0$ for all $x_{1}, y_{1} \in \mathfrak{g}_{1}$. Applying this to $y_{1} \in \mathfrak{g}_{1}^{\prime}$ and $x_{1} \in \mathfrak{g}_{1}^{\perp}=\left\{x_{1} \in \mathfrak{g}_{1} ; \rho\left(x_{1}, \mathfrak{g}_{1}^{\prime}\right)=0\right\}$ we get $D \mathfrak{g}_{1}^{\perp} \subset \mathfrak{g}_{1}^{\perp}$ (since $D \mathfrak{g}_{1}^{\prime} \subset \mathfrak{g}_{1}^{\prime}$ anyway). We also have $j \mathfrak{g}_{1}^{\perp} \subset \mathfrak{g}_{1}^{\perp}$. Finally, from (6) and (7) we know $D j x_{1}=j D x_{1}+g_{1}^{\prime}$, where $g_{1}^{\prime} \in \mathfrak{g}_{1}^{\prime}$. For $x_{1} \in \mathfrak{g}_{1}^{\perp}$ we obtain $g_{1}^{\prime} \in \mathfrak{f}$. If $x_{1} \in g_{\lambda}, \lambda \neq 0$, then $g_{1}^{\prime}=0$. If $x_{1} \in g_{0}$, then $g_{1}^{\prime}=0$, since $D$ commutes with $j$ in $g_{0}$, This finishes the proof of the lemma.

1.8. Let $\mathscr{D}$ be the closure of $\{\exp D ; D$ is the semisimple part of some adjn, $\left.n \in \hat{a}_{1}\right\}$ in $\mathrm{Gl}(\mathfrak{g})$. Then $\mathscr{D}$ is an abelian compact group of automorphisms of $\mathfrak{g}$. Moreover, $\mathscr{D}$ acts trivially on $\mathfrak{f}$ and commutes with ad $\mathfrak{f}$ $\mathfrak{a}$ by Corollary $1, \mathrm{c})$ of 1.4 and with $j$ by 1.7 . Also, if $x \notin \mathfrak{f}$ then $W x \notin \mathfrak{f}$ for all $W \in \mathscr{D}$.

We consider the skew from $\tilde{\rho}$ on $g$ given by

$$
\tilde{\rho}(u, v)=\int_{\mathscr{D}} \rho(W u, W v) d W,
$$

where $d W$ denotes the normalized bi-invariant Haar measure of $\mathscr{D}$.

It is easy to verify (3.4) through (3.7) of $[6 ; \S 3]$. Hence we have

Lemma. a) ( $\mathfrak{g}, \mathfrak{f}, j, \tilde{\rho})$ is a Kähler algebra.

b) The semisimple parts of ad $j n, n \in \hat{a}_{1}$, are skew-symmetric relative to $\tilde{\rho}$ and commute with $j$.

1.9. We want to consider modifications of $\mathfrak{g}$, for which ad $j n$ is 
nilpotent for all $n \in \mathfrak{n}$. The modification map has to vanish on [g, g]. We therefore prove

Lemma. After an inessential change of $j$ we may assume that $\operatorname{ad}(j \mathfrak{n} \cap[\mathfrak{g}, \mathfrak{g}])$ consists of nilpotent elements.

Proof. Suppose $j n \in[\mathfrak{g}, \mathfrak{g}]$ and denote by $D$ the semisimple part of ad $j n$, then $\operatorname{ad} j n \in[\operatorname{adg}, \operatorname{ad} g]=\mathfrak{m}$. But $\mathfrak{m}$ is an algebraic Lie algebra, whence $D \in \mathfrak{m}$. Hence, by the appendix we can write $D=D_{h}+D_{r},\left[D_{h}, D_{r}\right]$ $=0$ anc we can find a maximal semisimple subalgebra $\mathfrak{h}$ of $\mathfrak{g}$ and $h_{0} \in \mathfrak{h}$ such that ad $h_{0} D_{h}$. Moreover, $D_{r} \in \operatorname{rad}[\operatorname{adg}$, adg]. But this radical consists only of nilpotent endomorphisms, whence $D_{r}=0$. Clearly $h_{0} \in g_{0}$ where $g_{0}$ is defined in 1.6. If $D \neq 0$ we have $g_{0} \neq g$. Moreover, from 1.7 we can easily derive that $\mathfrak{g}_{0}$ is a Kähler algebra and $\mathfrak{g}_{0}=\left(\mathfrak{x} \cap \mathfrak{g}_{0}\right)+j\left(\mathfrak{x} \cap \mathfrak{g}_{0}\right)$ $+\mathfrak{f}$. We can apply the induction hypothesis to $\mathfrak{g}_{0}$. Since $\operatorname{ad} h_{0}$ is skew adjoint relative to $\tilde{\rho}$ we have $\tilde{\rho}\left(h_{0},[\mathfrak{g}, \mathfrak{g}]\right)=0$ by the closedness condition. This implies in particular $\tilde{\rho}\left(h_{0}, \mathfrak{h}+\operatorname{nil}(\mathfrak{g})\right)=0$. Let $\mathfrak{q}$ be a complement of $\operatorname{nil}(\mathfrak{g})$ in $\operatorname{rad} \mathfrak{g}$ which is $\mathfrak{h}$-invariant. Then $[\mathfrak{h}, \mathfrak{g}]=0$. But then $\tilde{\rho}(\mathfrak{q}, \mathfrak{h})=$ $\tilde{\rho}(\mathfrak{q},[\mathfrak{h}, \mathfrak{h}])=0$. In particular $\tilde{\rho}\left(\mathfrak{h}_{0}, \mathfrak{g}\right)=0$. Therefore $\tilde{\rho}\left(h_{0}, \mathfrak{g}\right)=0$. In particular $0=\tilde{\rho}\left(j h_{0}, h_{0}\right)$, whence $h_{0} \in \mathfrak{f}$. From $\left[h_{0}, \mathfrak{l}\right]=D \mathfrak{I}=0$ we get $h_{0} \in$ center(f). Now we introduce an inessential change of $j$ by redefining $j$ on a $\mathfrak{l}$-invariant complement $\mathfrak{b}_{1}$ of $\left[\mathfrak{f}, \mathfrak{n}_{1}\right]$ in $\mathfrak{n}_{1}$. For $n \in \mathfrak{b}_{1}$ we set $j^{\prime} n=j n$ $-h_{0}$. Then ad $j^{\prime} n$ is nilpotent for all $n \in \mathfrak{n}$ such that ad $j n \in$ ad $[\mathfrak{g}, \mathfrak{g}]$. We will also assume that the center of $\mathfrak{i}$ is $j^{\prime}$-invariant.

We claim that $\mathfrak{n}+j^{\prime} \mathfrak{n}$ is a solvable algebra having all properties of Corollary 1 of 1.4. We note $\hat{a}_{0}+\left[\mathfrak{f}, \hat{a}_{1}\right] \subset \mathfrak{n}+j^{\prime} \mathfrak{n}$ and $\left[\mathfrak{n}+j^{\prime} \mathfrak{n}, \mathfrak{n}+j^{\prime} \mathfrak{n}\right] \subset$ $\left[\mathfrak{a}+\mathfrak{f}_{0}, \mathfrak{a}+\mathfrak{l}_{0}\right]$ where $\mathfrak{f}_{0}$ denotes the center of $\mathfrak{l}$. Hence the last commutator is contained in $\hat{a}_{0}+\left[\mathfrak{f}_{0}, \mathfrak{a}\right]$. But $\left[\mathfrak{f}_{0}, \mathfrak{a}\right] \subset \hat{\mathfrak{a}}_{0}+\left[\mathfrak{f}, \hat{\mathfrak{a}}_{1}\right]$ and $\mathfrak{n}+j^{\prime} \mathfrak{n}$ is a solvable subalgebra of $\mathfrak{g}$. Moreover, $\left[\mathfrak{f}, \mathfrak{n}+\mathfrak{j}^{\prime} \mathfrak{n}\right] \subset\left[\mathfrak{\mathfrak { l }}, \mathfrak{a}+\mathfrak{f}_{0}\right]=[\mathfrak{f}, a] \subset \hat{a}_{0}$ $+\left[\mathfrak{f}, \hat{a}_{1}\right] \subset \mathfrak{n}+j^{\prime} \mathfrak{n}$ and $\left[k, j^{\prime} x\right]=\left[k, j x+k_{0}\right]=[k, j x]=j[k, x]=j[k, x]$ for all $x \in \mathfrak{n}+j^{\prime} \mathfrak{n}$ since ad[$[\mathfrak{f}, \mathfrak{a}]$ consists of nilpotent endomorphisms. Therefore, using $j^{\prime}$ instead of $j$ we may assume that $j n \cap[\mathfrak{g}, \mathfrak{g}]$ operates nilpotent]y on $g$. This proves the lemma.

1.10. From 1.9 we see that the elements of $\operatorname{ad}(j \mathfrak{n} \cap[\mathfrak{g}, \mathfrak{g}])$ have no semisimple parts. Denote by $\mathfrak{b}$ an algebraic complement of $j \mathfrak{n} \cap[\mathfrak{g}, \mathfrak{g}]$ in

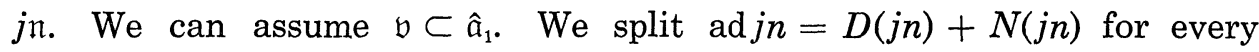
$j n \in \mathfrak{b}$ into semisimple part $D(j n)$ and nilpotent part $N(j n)$ and define a (modification) map $D: \mathfrak{g} \rightarrow \operatorname{Der} \mathfrak{g}$ by $D([\mathfrak{g}, \mathfrak{g}])=0$ and $D(\mathfrak{b})$ as above and 
trivial otherwise. It is easy to verify the properties (3.1) to (3.4) of [5] for the map $D$ and the Kähler algebra $(\mathfrak{g}, \mathfrak{f}, j, \tilde{\rho})$. Moreover, as $D(j n)$ is the semisimple part of ad $j n$ we know $D(j n) \mathfrak{g} \subset[\mathfrak{g}, \mathfrak{g}]$. Hence [5: 3.5] is satisfied.

We define a new algebra structure on $\mathfrak{g}$ by setting $[x, y]^{1}=[x, y]-$ $D(x) y+D(y) x, x, y \in \mathfrak{g}$.

A straightforward computation shows

Lemma. $\left(\mathfrak{g},[\cdot, \cdot]^{1}, \mathfrak{f}, j, \tilde{\rho}\right)$ is a Kähler algebra.

It is clear that $\mathfrak{r}$ and $\mathfrak{n}$ are also ideals of $\left(\mathfrak{g},[\cdot, \cdot]^{1}\right)$. The subalgebra $\mathfrak{n}+j \mathfrak{n}$ is now abelian and acts nilpotently on $\mathfrak{g}$. Moreover, it is easy to verify that the Radical Conjecture holds for $(\mathfrak{g},[\cdot, \cdot], \mathfrak{f}, j, \tilde{\rho})$ iff it holds for $\left(\mathfrak{g},[\cdot, \cdot]^{1}, \mathfrak{f}, j, \tilde{\rho}\right)$.

Therefore, from now on we assume (w.r.g.) that $\mathfrak{a}=\mathfrak{n}+j \mathfrak{n}$ is abelian and ad $a$ is nilpctent for all $a \in \mathfrak{a}$.

1.11. We want to follow the proof of $[5 ; \S 6]$. Therefore, we consider the set $\mathfrak{w}=\{x \in \mathfrak{g} ;$ ad $x \mid \mathfrak{n}$ and $\operatorname{ad} j x \mid \mathfrak{n}$ are skew adjoint relative to $\langle\cdot, \cdot\rangle$ $=\rho(j \cdot, \cdot)$ and commute with $j\}$. Put $\tilde{\mathfrak{w}}=\{x \in \mathfrak{w} ;[x, \mathfrak{n}]=0\}$. Clearly, $\mathfrak{w}$ is $j$-invariant (since $\mathfrak{f}$ is skewadjoint on $\mathfrak{a}$ and commutes with $j$.)

Lemma. $[\mathfrak{a}, \mathfrak{g}] \subset \mathfrak{\mathfrak { w }}$.

Proof. Note $[[j n, x], m]=[j n,[x, m]]=0$ for all $n, m \in \mathfrak{n}, x \in \mathfrak{g}$. Also $[j[j n, x], m]=[[j n, j x]-j[n, j x]-[n, x]-k, m]=-[k, m]$ for all $n, m \in \mathfrak{n}$. Hence $[j \mathfrak{n}, \mathfrak{g}] \subset \tilde{\mathfrak{w}}$. Clearly, $[\mathfrak{n}, \mathfrak{g}] \subset \mathfrak{n} \subset \tilde{\mathfrak{w}}$. Hence the lemma.

\subsection{2 .}

LEMMA. $\rho([\mathfrak{a}, \tilde{\mathfrak{w}}], \mathfrak{a})=0$.

Proof. Let $w \in \tilde{\mathfrak{w}}$ and $n, n^{1}, m, m^{1} \in \mathfrak{n}$. Then $\rho\left(\left[n^{1}+j n, w\right], m^{1}+j m\right)$ $=\rho\left([j n, w], m^{1}+j m\right)=-\rho\left(\left[w, m^{1}+j m\right], j n\right)=-\rho([w, j m], j n)$ where we have used the fact that $\mathfrak{a}$ is abelian. Using the integrability condition for $\mathfrak{g}$ we get $\rho([w, j m]), j n)=-\rho(j[w, j m], n)=-\rho([j w, j m]-j[j w, m]-[w, m]$ $-k, n)=-\rho([j w, j m], n)-\rho([j w, m], j n)=\rho([n, j w], j m)-\rho([j w, m], j n)=A$. Set $S(j w)=\operatorname{ad} j w \mid \mathfrak{n}$. Then we get $A=-\rho(S(j w) n, j m)-\rho(S(j w) m, j n)$ $=-\rho(S(j w) n, j m)+\rho(j S(j w) m, n)=0$ because $S(j w)$ is skewadjoint relative to $\langle n, m\rangle=\rho(j n, m)$.

Corollary. $\rho\left((\operatorname{ad} \mathfrak{a})^{2} \mathfrak{g}, \mathfrak{a}\right)=0$. 
1.13. Using the above corollary we see

LEMmA. $\quad \rho\left(e^{t \text { ad } h} x, e^{t \text { ad } h} y\right)=a t^{2}+b t+c$ for $h \in \mathfrak{a}, x, y \in \mathfrak{g}$ and some $a, b, c \in R$.

Proof. We know $\frac{d^{3}}{d t^{3}} \rho\left(e^{t \operatorname{ad} h} x, e^{t \operatorname{ad} h} y\right)=\frac{d^{2}}{d t^{2}} \rho\left(h, e^{t \operatorname{ad} h}[x, y]\right)=\rho\left(h,(\operatorname{ad} h)^{2 *}\right)$ $=0$. Hence the lemma.

1.14. Next we want to prove the following easy

Lemma. $(\operatorname{ad} a)^{r} j x=j(\operatorname{ad} a)^{r} x \bmod \mathfrak{g}^{\prime}$ for all $a \in \mathfrak{a}, x \in \mathfrak{g}$.

Proof. The claim is trivial for $r=0$. For $r=1$ we split $a=n^{\prime}+j n$, $n, n^{\prime} \in \mathfrak{n}$ and see that it suffices to consider $a=j n$. But here the claim follows immediately from the integrability condition. Let now $r \geq 2$. Then $(\operatorname{ad} a)^{r} j x=(\operatorname{ad} a)(\operatorname{ad} a)^{r-1} j x=(\operatorname{ad} a)\left\{j(\operatorname{ad} a)^{r-1} x+g^{\prime}\right\}=j(\operatorname{ad} a)^{r} x+g^{\prime \prime}$ with some $g^{\prime}, g^{\prime \prime} \in \mathfrak{g}^{\prime}$.

1.15. The trivial results above can be combined to give.

Lemma. $(\mathrm{ad} \mathfrak{a})^{2} \mathfrak{g} \subset \mathfrak{f}$.

Proof. $\quad b t^{2}+c t+d=\rho\left(e^{t \operatorname{ad} a}[a,[a, x]], \quad e^{t \operatorname{ad} a} j[a, y]\right)=\rho\left(e^{t a d a}[a,[a, x]]\right.$, $\left.j e^{\operatorname{tad} a}[a, y]+g^{1}\right)=-\left\langle e^{\operatorname{tad} a}[a,[a, x]], e^{t \operatorname{ad} a}[a, y]\right\rangle$ where $x, y \in \mathfrak{g}, a \in a$. With $y=[a, x]$ we see that $B=\left|e^{t \operatorname{ad} a}(\operatorname{ad} a)^{2} x\right|^{2}$ is a quadratic polynomial in $t$. We know that ad $a$ is nilpotent, hence $B$ is a polynomial in $t$. Let ad $a$ be nilpotent of degree $s$, i.e. $(\operatorname{ad} a)^{s}=0,(\operatorname{ad} a)^{s-1} \neq 0$. Then $B$ is a polynomial of degree $\leq 2(s-3)$ and the coefficient of $t^{2(s-3)}$ is $\left|(\operatorname{ad} a)^{s-1} x\right|^{2}$. If $2(s-3) \leq 2$, then $s \leq 4$. If $2(s-3)>2$, then $(\operatorname{ad} a)^{s-1} \mathfrak{g} \subset \mathfrak{f}$ and the highest term in $B$ is at most of degree $2(s-4)$. Suppose also $2(s-4)>2$, then by the same argument as above, $(\operatorname{ad} a)^{s-2} g \subset \mathfrak{f}$. But then $(\operatorname{ad} a)^{s-1} \mathfrak{g} \subset$ $[a, f] \cap f=0$. Therefore $2(s-4) \leq 2$, i.e. $s \leq 5$. As seen above, this implies $(\operatorname{ad} a)^{4} \mathrm{~g} \subset \mathfrak{f}$. Hence in both cases, $2(s-3) \leq 2$ and $2(s-3)>2$, we have $(\operatorname{ad} a)^{4} \mathrm{~g} \subset \mathfrak{k}$. Choosing $y=x$ above and again comparing highest terms in $t$ we get even $(\operatorname{ad} a)^{3} \mathfrak{g} \subset \mathfrak{f}$. Finally, we consider $C=-\left\langle e^{t \operatorname{ad} a}[a, x], e^{t \operatorname{ad} a} x\right\rangle$ $=\rho\left([a, x]+t[a,[a, x]], j x+t j[a, x]+(1 / 2) t^{2} j[a,[a, x]]\right.$. We know $j[a, x]=$ $[a, j x]+g^{\prime}$ and $j(\operatorname{ad} a)^{2} x=(\operatorname{ad} a)^{2} j x+g^{\prime \prime}$ where $g^{\prime}, g^{\prime \prime} \in g^{\prime}$. We note that the coefficient of $t^{3}$ equals $-(1 / 2)\left|(\operatorname{ad} a)^{2} x\right|^{2}=(1 / 2) \rho\left((\operatorname{ad} a)^{2} x,(\operatorname{ad} a)^{2} j x\right)$ by Corollary 1.12. Hence, altogether, we get $C=\rho\left(e^{t \mathrm{ad} a}[a, x], e^{t \mathrm{ad} a} j x\right)+p(t)$ where $p(t)$ is a quadratic polynomial. Applying Lemma 1.13 we see that actuaily $C$ is a quadratic polynomial. Therefore $(\operatorname{ad} a)^{2} \mathfrak{g} \subset \mathfrak{f}$. 
1.16. Later we will frequently use the following.
LEMma. a) $a \subset \operatorname{rad}(\mathfrak{g})$
b) $[\mathfrak{a}, \mathfrak{g}] \subset \operatorname{nil}(\mathfrak{g})$.
c) $(\mathrm{ad} \mathfrak{a})^{2}=0$.

Proof. It suffices to prove a) for an element of type $j n, n \in \mathfrak{n}$. We work in $\tilde{\mathrm{g}}=\mathrm{g} / \mathrm{radg}$. Suppose $\operatorname{ad} j n \neq 0$ on $\tilde{\mathrm{g}}$. Then $0 \neq \tilde{x}=j n \bmod \operatorname{rad}(g)$ is nilpotent in the semisimple algebra $\tilde{g}$. Therefore, by the JacobsonMorozov-Theorem there exist $\tilde{y}, \tilde{h} \in \tilde{\mathfrak{g}}$ such that $\boldsymbol{R} \tilde{x}+\boldsymbol{R} \tilde{h}+\boldsymbol{R} \tilde{y} \cong \operatorname{sl}(2, \boldsymbol{R})$. In particular $(\operatorname{ad} \tilde{x})^{2} \tilde{y}=2 \tilde{x}$. On the other hand we know $(\operatorname{adj} n)^{2} \mathfrak{g} \subset \mathfrak{f}$ $\bmod \operatorname{rad}(\mathfrak{g})$. Hence $\tilde{x} \in \mathfrak{I} \bmod \operatorname{rad}(\mathfrak{g})$. Since $\mathfrak{l}$ acts semisimple on $\mathfrak{g}$ (and on $\tilde{\mathrm{g}}$ ) and $\tilde{x}$ acts nilpotent, $\tilde{x}=0$. This is a contradiction and $\tilde{x}=0$ follows, whence a). Part b) is a trivial consequence of a). To prove c) we combine $\mathfrak{a} \subset \operatorname{rad}(\mathfrak{g})$ with Lemma 1.15 and $\operatorname{get}(\operatorname{ad} \mathfrak{a})^{2} \mathfrak{g} \subset \operatorname{nil}(\mathfrak{g}) \cap \mathfrak{l}=0$.

1.17. Eventually we want to generalize the proof of $[5 ; \S 6]$ to our setting. We therefore consider $\check{\mathfrak{g}}^{(1)}=[j \mathfrak{n}, \mathfrak{g}]+\mathfrak{g}^{\prime}$. Note $[j \mathfrak{n}, \mathfrak{g}] \subset \operatorname{nil}(\mathfrak{g})$ by 1.16 .

LEMmA. a) $\check{\mathfrak{g}}^{(1)}$ is a Kähler algebra.

b) $\breve{\mathfrak{g}}^{(1)}=\left(\mathfrak{x} \cap \check{\mathfrak{g}}^{(1)}\right)+j\left(\mathfrak{x} \cap \check{\mathfrak{g}}^{(1)}\right)+\mathfrak{\mathfrak { l }}$.

Proof. a) Clearly $\left[\mathfrak{n}, \breve{\mathfrak{g}}^{(1)}\right] \subset \check{\mathfrak{g}}^{(1)}$. Because $(\operatorname{ad} j n)^{2}=0$, we also have $\left[j \mathfrak{n}, \check{\mathfrak{g}}^{(1)}\right] \subset\left[j \mathfrak{n}, \mathfrak{g}^{\prime}\right] \subset \mathfrak{g}^{\prime} \subset \check{\mathfrak{g}}^{(1)}$. For $k \in \mathfrak{I}$ we get $[k,[j \mathfrak{n}, \mathfrak{g}]] \subset[j[k, \mathfrak{n}], \mathfrak{g}]+$ $[j \mathfrak{n}, \mathfrak{g}] \subset \breve{\mathfrak{g}}^{(1)}$. Finally $[[j n, x],[j m, y]]=[j n,[x,[j m, y]]] \in \breve{\mathfrak{g}}^{(1)}$ because $(\operatorname{ad} j \mathfrak{n})^{2}$ $=0$. This shows that $\check{\mathfrak{g}}^{(1)}$ is a subalgebra of $\mathfrak{g}$. The integrability condition implies that $\breve{\mathrm{g}}^{(1)}$ is $j$-invariant.

b) We have $[j n, j r]+j[j n, r] \bmod g^{\prime}$. Therefore $[j \mathfrak{n}, \mathfrak{r}]+\mathfrak{n}+j([j \mathfrak{n}, \mathfrak{x}]$ $\mathfrak{n})+\mathfrak{l}=\breve{\mathfrak{g}}^{(1)}$. From this the assertion follows.

1.18. From the definition of $\check{\mathfrak{g}}^{(1)}$ it follows $\breve{g}^{(1)} \subset \operatorname{nil}_{0}(\mathfrak{g})+\mathfrak{l}$, where $\operatorname{nil}_{0}(\mathfrak{g})$ denotes the greatest nilpotent ideal of $g[2 ; \S 4.4$ and $\S 5.3]$. We may assume that $\mathfrak{a}^{(1)}=\breve{\mathfrak{g}}^{(1)} \cap \operatorname{nil}_{0}(\mathfrak{g})$ is invariant under $j$. In particular, as ad $\mathfrak{a}^{(1)}$ consists only of nilpotent derivations of $\mathfrak{g}$, we see that $\mathfrak{a}^{(1)}$ is abelian.

We would like to point out that $\mathfrak{a}^{(1)}=[j \mathfrak{n}, \mathfrak{g}]+\mathfrak{n}+j \mathfrak{n} \subset \operatorname{nil}_{0}(\mathfrak{g})$ holds.

1.19. Next we want to consider $\mathfrak{g}^{(0)}=\left\{x \in \mathfrak{g} ;\left[x, \mathfrak{g}^{(2)}\right] \subset \mathfrak{g}^{(2)}\right\}$ where $\mathfrak{g}^{(2)}$ $=\mathfrak{n}+j \mathfrak{n}$. Before we can show that $\mathfrak{g}^{(0)}$ satisfies the induction hypothesis we have to consider the vector space $\mathfrak{r} \cap j \mathrm{r}$.

Let $\mathfrak{b}=\{x \in \mathfrak{x} ; j x \in \mathfrak{r}+\mathfrak{t}\}$. 
After an inessential change of $j$ we can assume $j x \in \mathfrak{x}$ for all $x \in \mathfrak{v}$. We note that this can be done without changing $j$ on $\mathfrak{x} \cap \check{g}^{(1)}$.
LEMMA.
a) $\mathfrak{v}=\mathfrak{x} \cap j \mathfrak{x}$
b) $j \mathfrak{b} \subset \mathfrak{b}$

Proof. Let $x \in \mathfrak{v}$, then $x, j x \in \mathfrak{x}$ and $j(j x)=-x+k$. Therefore $j x \in \mathfrak{v}$. But then $j(j x) \in \mathfrak{x}$, whence $k \in \mathfrak{x}$ and $k=0$. Hence $x=j(-j x) \in \mathfrak{x} \cap j \mathfrak{x}$ and $\mathfrak{b} \subset \mathfrak{r} \cap j \mathfrak{x}$. Let $x \in \mathfrak{r} \cap j \mathfrak{r}$; then $x \in \mathfrak{r}$ and $x=j y$ for some $y \in \mathfrak{r}$. Hence $j x$ $=-y+k$ and $x \in \mathfrak{v}$ follows. This proves the lemma.

1.20. Lmma. $\mathfrak{x} \cap j \mathfrak{x}+\mathfrak{n}$ is an ideal of $\mathfrak{g}$.

Proof. We have to verify that $\mathfrak{b}$ is mapped into $\mathfrak{v}+\mathfrak{n}$ by $\mathfrak{f}, \mathfrak{x}$ and $j \mathfrak{x}$. Let $x \in \mathfrak{v}=\mathfrak{x} \cap j \mathfrak{r}$ and $y \in \mathfrak{r}$ such that $x=j y$ holds. Clearly $[\mathfrak{f}, x] \subset \mathfrak{r}$; moreover, $[k, j y]=j[k, y]+k^{\prime}$ by the definition of a Kähler algebra. Hence $j[k, y]+k^{\prime} \in \mathfrak{x}$, yielding $[k, y] \in \mathfrak{v}$. Therefore $k^{\prime}=0$ and $[k, x]=j[k, y] \in \mathfrak{x} \cap j \mathfrak{r}$. Next note $[\mathfrak{r}, \mathfrak{x}] \subset \mathfrak{n}$. Finally, we consider [jr, $\mathfrak{b}]$. Then, with $x$ and $y$ as above, we have $[j r, j y]=j([j r, y]+[r, j y])+[r, y]+k \in \mathfrak{r}$. Because $[r, y]$ $\in \mathfrak{n}$, we obtain $j z+k \in \mathfrak{x}$, where $z=[j r, y]+[r, j y] \in \mathfrak{x}$. This yields $z \in \mathfrak{b}$ and $k=0$. Therefore $[j r, j y] \in j \mathfrak{v}+n \subset \mathfrak{v}+\mathfrak{n}$. This finishes the proof.

1.21. From the last lemma we see that $\mathfrak{n} \subset \mathfrak{v}+\mathfrak{n} \subset \mathfrak{x}$ is a chain of ideals. We had chosen $\mathfrak{n}$ maximal. Therefore either $\mathfrak{r} \cap j \mathfrak{x} \subset \mathfrak{n}$ or $\mathfrak{x} \cap j \mathfrak{x}$ $+\mathfrak{n}=\mathfrak{x}$. In the latter case we get $\mathfrak{g}=\mathfrak{x} \cap j \mathfrak{x}+\mathfrak{n}+j \mathfrak{n}+\mathfrak{f}$. Hence $\mathfrak{g}=$ $\operatorname{rad} \mathfrak{g}+\mathfrak{f}$. After an inessential change of $j$ we may assume that rad $\mathfrak{g}$ is $j$-invariant and the Radical Conjecture holds.

Hence, from now on we will assume $\mathfrak{x} \cap j \mathfrak{x} \subset \mathfrak{n}$.

1.22. Now we can return to the consideration of the subalgebra $g^{(0)}$ $=\left\{x \in \mathfrak{g} ;\left[x, \mathfrak{g}^{(2)}\right] \subset \mathfrak{g}^{(2)}\right\}$ where $\mathfrak{g}^{(2)}=\mathfrak{n}+j \mathfrak{n}$. Clearly, $\mathfrak{g}^{\prime} \subset \mathfrak{g}^{(0)}$.

Lemma. a) $\mathrm{g}^{(0)}$ is a Kähler subalgebra of $\mathrm{g}$.

b) $\mathfrak{g}^{(0)}=\left(\mathfrak{g}^{(0)} \cap \mathfrak{x}\right)+j\left(\mathfrak{g}^{(0)} \cap \mathfrak{x}\right)+\mathfrak{l}$.

Proof. a) Let $x \in \mathfrak{g}^{(0)}$. Then we have to prove that $j x$ leaves $\mathfrak{g}^{(2)}$ invariant. This follows from $[j x, \mathfrak{n}] \subset \mathfrak{n} \subset \mathfrak{g}^{(2)}$ and $[j x, j n]=j[j x, n]+$ $j[x, j n]+[x, n]+k$; note that in the last expression all summands but $k$ are in the greatest nilpotent ideal of $\mathrm{g}$. Therefore ad $k=0$. Hence $k=0$, because we have assumed that $\mathfrak{g}$ acts effectively on some manifold. But this implies $[j x, j n] \in \mathrm{g}^{(2)}$. 
b) Because $\subset \mathrm{g}^{(0)}$ we have only to consider elements of type $x=\tilde{r}$ $+j r \in \mathfrak{g}^{(0)}$. Then $[j n, x] \in \mathfrak{g}^{(2)}$, i.e. $[j n, \tilde{r}]+[j n, j r]=[j n, \tilde{r}]+j[j n, r]+j[n, j r]$ $+[n, r]+k \in \mathfrak{g}^{(2)}$. Therefore $[j n, \tilde{r}]+j[j n, r] \in \mathfrak{g}^{\prime}$. Hence there exist $a, b$ $\in \mathfrak{n}, k^{\prime} \in \mathfrak{f}$ such that $[j n, \tilde{r}]+j[j n, r]=a+j b+k^{\prime}$. This implies $j([j n, r]-b)$ $=a-[j n, \tilde{r}]+k^{\prime}$, whence $[j n, r]-b \in \mathfrak{b}$ and $k^{\prime}=0$. In particular [jn, r] $-b \in \mathfrak{n}$. Hence we obtain $[j n, r],[j n, \tilde{r}] \in \mathfrak{n}$. This implies $\left[r, \mathfrak{g}^{(2)}\right] \subset \mathfrak{g}^{(2)}$ and $\left[\tilde{r}, \mathfrak{g}^{(2)}\right] \subset \mathfrak{g}^{(2)}$, whence $r, \tilde{r} \in \mathfrak{g}^{(0)}$. We have shown $\mathfrak{g}^{(0)} \subset\left(\mathfrak{g}^{(0)} \cap \mathfrak{r}\right)+j\left(\mathfrak{g}^{(0)}\right.$ $\cap \mathfrak{x})+\mathfrak{f}$. The opposite inclusion is trivial and the lemma is proven.

1.23. To be able to use the induction hypothesis for $\mathfrak{g}^{(0)}$ we have to exclude the case $\mathfrak{r} \cap \mathfrak{g}^{(0)}=\mathfrak{r}$. But in this case $\mathfrak{g}=\mathfrak{g}^{(0)}$ and $\mathfrak{g}^{(2)}$ is an abelian ideal of $\mathfrak{g}$. Consider $\mathfrak{u}=\left\{x \in \mathfrak{g}, \rho\left(x ; \mathfrak{g}^{(2)}\right)=0\right\}$. Then $\mathfrak{u}$ is a Kähler subalgebra of $\mathfrak{g}$ and $\mathfrak{u} \cap \mathfrak{g}^{(2)}=0$. Clearly $\mathfrak{u} \cong \mathfrak{g} / \mathfrak{g}^{(2)}$. Therefore we can apply the induction hypothesis to $\mathfrak{u}$. So after an inessential change of $j$ on $\mathfrak{u}$ we get a solvable Kähler subalgebra $\tilde{\mathfrak{s}}$ of $\mathfrak{u}$ satisfying $\mathfrak{u}=\tilde{\mathfrak{g}}+\mathfrak{f}, \tilde{\mathfrak{v}} \cap \mathfrak{f}=0$. But the the Radical Conjecture follows with $\mathfrak{z}=\mathfrak{g}^{(2)}+\tilde{\mathfrak{s}}$.

Hence, from now on we will assume $\mathrm{g}^{(0)} \neq \mathrm{g}$. From the last lemma it follows that we can apply the induction hypothesis. So after an inessential change of $j$ we have $\mathfrak{g}^{(0)}=\mathfrak{t}^{(0)}+\mathfrak{a}^{(0)}+\mathfrak{f}$, where $\mathfrak{t}^{(0)}+\mathfrak{a}^{(0)}$ is a solvable Kähler algebra, $t^{(0)}$ is a modification of a normal $j$-algebra and $\mathfrak{a}^{(0)}$ is a modification of an abelian Kähler algebra.

From 1.18 we know $\mathfrak{a}^{(1)} \subset \operatorname{nil}_{0}(\mathfrak{g}) \cap \mathfrak{g}^{(0)}$. Therefore also $\mathfrak{a}^{(1)}+\left[\mathfrak{a}^{(1)}, \mathfrak{g}\right] \subset$ $\operatorname{nil}_{0}(\mathfrak{g}) \cap \mathfrak{g}^{(0)} \subset \operatorname{nil}_{0}\left(\mathfrak{g}^{(0)}\right)$. It is easy to see that one can choose $\mathfrak{t}^{(0)}+\mathfrak{a}^{(0)}$ so that $\operatorname{nil}_{0}\left(\mathfrak{g}^{(0)}\right) \subset \mathfrak{t}^{(0)}+\mathfrak{a}^{(0)}$ holds.

LEMMA. a) $\mathfrak{a}^{(1)} \subset \mathfrak{a}^{(0)}$.

b) $\check{\mathfrak{g}}^{(1)}$ is a subalgebra of $\mathrm{g}^{(0)}$.

Proof. It suffices to prove $[j \mathfrak{n}, \mathfrak{g}] \subset \mathfrak{g}^{(0)}$. It is easy to səe that we only have to note $[j \mathfrak{n},[j \mathfrak{n}, \mathfrak{g}]]=0 \subset \mathfrak{g}^{(2)}$.

1.24. We want to generalize the proof of $[5 ; \S 6]$ to our setting. We define $\mathfrak{g}^{(-1)}=\mathfrak{g}, \mathfrak{g}^{(0)}=\mathfrak{t}^{(0)}+\mathfrak{a}^{(0)}+\mathfrak{f}, \mathfrak{g}^{(1)}=\mathfrak{a}^{(1)}$ and $\mathfrak{g}^{(2)}=\mathfrak{n}+j \mathfrak{j}$.

We have seen above $\mathfrak{g}^{(-1)} \supset \mathfrak{g}^{(0)} \supset \mathfrak{g}^{(1)} \supset \mathfrak{g}^{(2)}$. Also, $j \mathfrak{g}^{(k)} \subset \mathfrak{g}^{(k)}$. By the definition of $\mathfrak{g}^{(1)}$ and $\mathfrak{g}^{(0)}$ we have $\left[\mathfrak{g}^{(-1)}, \mathfrak{g}^{(2)}\right] \subset \mathfrak{g}^{(1)}$ and $\left[\mathfrak{g}^{(0)}, \mathfrak{g}^{(2)}\right] \subset \mathfrak{g}^{(2)}$. Further we know $\left[\mathfrak{g}^{(1)}, \mathfrak{g}^{(1)}\right]=0$ and $\left[\mathfrak{g}^{(0)}, \mathfrak{g}^{(0)}\right] \subset \mathfrak{g}^{(0)}$ hence, in particular $\left[\mathfrak{g}^{(1)}, \mathfrak{g}^{(2)}\right]=0$.

LEMMA. The subspaces $\mathrm{g}^{(i)}$ form a j-invariant filtration of the Lie algebra g. 
Proof. We have only to consider two types of commutators.

$\left[\mathfrak{g}^{(0)}, \mathfrak{g}^{(1)}\right] \subset \mathfrak{g}^{(1)}$ : let $x_{0} \in \mathfrak{g}^{(0)}$ and $x_{1} \in \mathfrak{g}^{(1)}$. Because $\left[\mathfrak{g}^{(0)}, \mathfrak{g}^{(2)}\right] \subset \mathfrak{g}^{(2)} \subset \mathfrak{g}^{(1)}$ we can assume $x_{1}=[j n, x]$ for some $n \in \mathfrak{n}, x \in \mathfrak{g}$. Then $\left[x_{0}, x_{1}\right]=\left[x_{0},[j n, x]\right]=$ $\left[j n,\left[x_{0}, x\right]\right]-\left[\left[j n, x_{0}\right], x\right] \in \mathfrak{g}^{(1)}$.

$\left[\mathfrak{g}^{(-1)}, \mathfrak{g}^{(1)}\right] \subset \mathfrak{g}^{(0)}$ : let $x \in \mathfrak{g}$ and $x_{1} \in \mathfrak{g}^{(1)}$. Then for all $n \in \mathfrak{n}$ we have $\left[j n,\left[x, x_{1}\right]\right]=\left[[j n, x], x_{1}\right]+\left[x,\left[j n, x_{1}\right]\right]=0$ since $g^{(1)}$ is abelian. This proves the claim.

1.25. From this point on we can use large parts of the proof of [5; $\S 6]$. We have the obvious identifications: $\mathfrak{g}^{(i)} \leftrightarrow \mathfrak{g}^{(i)}, \mathfrak{a}^{(0)} \leftrightarrow \mathfrak{a}, \mathfrak{t}^{(0)} \leftrightarrow \mathfrak{J}_{D}$ $=\mathfrak{h}$ and $\mathfrak{n} \leftrightarrow \mathfrak{g}$.

(1) Let $x \in \mathfrak{a}^{(0)}$ and ad $x=S+N$ be the decomposition of ad $x$ into semisimple part $S$ and nilpotent part $N$. Then $N g \subset \mathfrak{g}^{(0)}$.

Proof. See [5].

(2) We denote by $\&$ the principal idempotent of $\mathfrak{t}^{(0)}$ and define $H_{0}$, $\mathfrak{g}_{\lambda}, \overline{\mathfrak{g}}, \overline{\mathfrak{g}}^{(\mu)} \ldots$ as in [5]. For an arbitrary $n \in \mathfrak{n}$ we define (as in [5]) $\{a b c\}=$ $[[[\overline{j n}, a], b], c], a, b, c \in \overline{\mathfrak{g}}^{(-1)}$.

(3) $\{a b c\}$ is invariant under permutations of $a, b, c$.

Proof. As in [7] one notes $\{a b c\}-\{b c a\}=[[[\overline{j n}, a], b], c]-[[[\overline{j n}, b], a], c]$ $=[[\overline{j n},[a, b]], c]=0$ since $\left[\bar{g}^{(-1)}, \bar{g}^{(-1)}\right]=0$. Hence $\{a b c\}=\{b a c\}$. Moreover, $[[[\overline{j n}, a], b], c]-[[[\overline{j n}, a], c], b]=[[\overline{j n}, a],[b, c]]=0$.

We also have

(4) $[\overline{j m},\{a b c\}]=[[[\overline{j n}, a], b],[\overline{j m}, c]]$ for all $m \in \mathfrak{n}$.

(5) $\bar{H}_{0} \bar{j}_{\alpha}=-\alpha \bar{j}_{\alpha}$ where $\alpha \in\{0, \pm 1 / 2\}, n_{\alpha} \in \mathfrak{n}_{\alpha}$.

The standard argument yields:

(6) $\left[j n_{\alpha}, \mathfrak{g}_{\lambda}^{(-1)}\right] \subset \mathfrak{g}_{\lambda-\alpha}^{(1)}$.

This implies

(7) $\lambda \in\{\alpha, \alpha \pm 1 / 2\}$ where $\alpha \in\{0, \pm 1 / 2\}$ if $\left[j n_{\alpha}, \mathfrak{g}_{\lambda}^{(-1)}\right] \neq 0$. Eventually we want to prove $\{a b c\}=0$ for all $n \in \mathfrak{n}$. It clearly suffices to prove this for $\mathfrak{n}=n_{\alpha} \in \mathfrak{n}_{\alpha}, \alpha \in\{0, \pm 1 / 2$.$\} . If j n \in \mathfrak{t}$, then $\{a b c\}=0$. This implies that we can assume $\alpha \in\{0,1 / 2\}$ since for $\alpha=-1 / 2$ we have $j n=[s, n] \in \mathfrak{n}$.

Suppose now that we can choose $a \in \overline{\mathfrak{g}}_{\lambda}^{(-1)}, b \in \overline{\mathfrak{g}}_{\mu}^{(-1)}, c \in \overline{\mathfrak{g}}_{\nu}^{(-1)}$, so that $\{a b c\} \neq 0$. From the definition and the symmetry of $\{a b c\}$ we derive that the commutators $\left[j n_{\alpha}, a\right],\left[j n_{\alpha}, b\right]$ and $\left[j n_{\alpha}, c\right]$ do not vanish. Hence, by (7),

(8) $\lambda, \mu, \nu \in\{\alpha, \alpha \pm 1 / 2\}$ where $\alpha \in\{0,1 / 2\}$.

The argument of [5] carries over without change to prove

(9) $\lambda+\mu, \mu+\nu, \nu+\lambda \in\{\alpha, 1+\alpha, 1 / 2+\alpha\}$, where $\alpha \in\{0,1 / 2\}$. 
Next we prove a result similar to $[5 ; \S 6.15]$.

$$
\left[\mathfrak{g}, \mathfrak{g}^{(1)}\right] \subset\left\{a \in \mathfrak{a}^{(0)} ; \operatorname{ad} a \mid \mathfrak{a}^{(0)}+\mathfrak{t}^{(0)} \text { is nilpotent }\right\}+\left[\mathfrak{t}^{(0)}, \mathfrak{t}^{(0)}\right] .
$$

Proof. Let $x \in\left[\mathfrak{g}, \mathfrak{g}^{(1)}\right]$. Then $x \in \operatorname{nil}_{0}\left(\mathfrak{g}^{(0)}\right) \subset \mathfrak{g}^{(0)}=\mathfrak{t}^{(0)}+\mathfrak{a}^{(0)}$ by the convention 1.23. Hence $\left[\mathfrak{g}^{(0)}, x\right] \subset \mathfrak{g}^{(0)}$. We write $x=t+a$; since $a d x \mid \mathfrak{g}^{(0)}$ is nilpotent, $t \in\left[\mathfrak{t}^{(0)}, \mathfrak{t}^{(0)}\right]$ and $\operatorname{ad} a \mid \mathfrak{F}^{(0)}$ is nilpotent. This proves the claim.

The next result is $[5 ; 6.16]$ and is proven as there. Let

(11) Let $g_{\nu} \in g_{\nu}, u_{\eta} \in \bar{g}_{\eta}^{(1)}, v_{\xi} \in \bar{g}_{\xi}^{(1)}$ and assume

$$
\eta+\xi>0 \text { or } \eta=\xi=0 \text {, then }\left[\left[g_{\nu}, u_{\eta}\right], v_{\xi}\right]=0 \text {. }
$$

This implies

$$
\left[\left[\bar{g}^{(-1)}, \bar{g}_{\eta}^{(1)}\right], \bar{g}_{\xi}^{(1)}\right]=0 \text { if } \eta+\xi>0 \text { or } \eta=\xi=0 .
$$

We want to apply (12) to $[\overline{j m},\{a b c\}]$. First we note that there exists some $m=m_{\beta} \in \mathfrak{n}_{\beta}$ so that $Q=[\overline{j m},\{a b c\}] \neq 0$; otherwise all representatives of $\{a b c\}$ are in $\mathrm{g}^{(0)}$, whence $\{a b c\}=0$. We note that $Q$ is symmetric in $a, b, c$ and in $n, m$. Therefofre $\beta \neq-1 / 2$. Moreover, (8) and (9) hold for $B$ as well and comparing (4) and (12) we see that

$$
(\lambda-\alpha)+(\nu-\beta) \leq 0 \text { and } \lambda-\alpha \neq 0 \text { or } \nu-\beta \neq 0 \text {. }
$$

The same relations hold for every permutation of $\lambda, \mu, \nu$ and transposition of $\alpha, \beta$.

If $\alpha \neq \beta$, then we can assume $\alpha=0, \beta=1 / 2$. In this case relations (8), (9) and (13) become

$$
\begin{aligned}
& (8)^{\prime} \quad \lambda, \mu, \nu \in\{0, \pm 1 / 2\} \cap\{0,1 / 2,1\}=\{0,1 / 2\} \\
& (9)^{\prime} \quad \lambda+\mu, \mu+\nu, \lambda+\nu \in\{0,1 / 2,1\} \cap\{1 / 2,1,3 / 2\}=\{1 / 2,1\} \\
& (13)^{\prime} \quad \lambda+u, \mu+\nu, \lambda+\nu \leq 1 / 2 .
\end{aligned}
$$

Hence $\lambda+\mu=\mu+\nu=\lambda+\nu=1 / 2$. Using (8)' we get a contradiction. Therefore, we can assume $\alpha=\beta \in\{0,1 / 2\}$. For $\alpha=0$ the relations (8), (9), (13) give

$$
\begin{array}{ll}
(8)^{\prime \prime} & \lambda, \mu, \nu \in\{0, \pm 1 / 2\} \\
(9)^{\prime \prime} & \lambda+\mu, \mu+\nu, \lambda+\nu \in\{0,1 / 2,1\} \\
(13)^{\prime \prime} & \lambda+\mu, \mu+\nu, \lambda+\nu \leq 0 .
\end{array}
$$

Hence $\lambda+\mu, \mu+\nu, \lambda+\nu=0$. Therefore $\mu=-\lambda$ and $\nu=-\mu=\lambda$. Since (13) also implies $\lambda \neq 0$ or $\nu \neq 0, \lambda, \mu, \nu \neq 0$. Therefore $\lambda+\nu=2 \lambda \neq 0$, a 
contrradiction.

Finally, for $1 / 2$ we get

$(8)^{\prime \prime \prime} \quad \lambda, \mu, \nu \in\{0,1 / 2,1\}$

$(9)^{\prime \prime \prime} \quad \lambda+\mu, \mu+\nu, \lambda+\nu \in\{1 / 2,1,3 / 2\}$

(13)"' $\lambda+\mu, \mu+\nu, \lambda+\nu \leq 1$ and at most one of the numbers $\lambda, \mu, \nu$ is equal to $1 / 2$.

This implies in particular that 1 is not attained in (13)"' . Therefore $\lambda+\mu$ $=\mu+\nu=\lambda+\nu=1 / 2$. Hence $\lambda, \mu, \nu \in\{0,1 / 2\}$. By $(13)^{\prime \prime \prime}$ two of these numbers have to be 0 , but then their sum is not $1 / 2$, a contradiction. Therefore we have proven altogether

$$
\{a b c\}=0 \text {. }
$$

This implies

$$
\left[\left[\mathfrak{g}^{(1)}, \mathfrak{g}\right], \mathfrak{g}\right] \subset \mathfrak{g}^{(0)}
$$

From this one derives as in [5]

$$
\left[\left[\mathfrak{g}^{(1)}, \mathfrak{g}\right], \mathfrak{g}^{(1)}\right]=0 \text {. }
$$

1.26. We consider $z=Z\left(\mathfrak{g}^{(1)}\right)=\left\{x \in \mathfrak{g} ;\left[x, \mathfrak{g}^{(1)}\right]=0\right\}$. Because $\mathfrak{g}^{(2)} \subset \mathfrak{g}^{(1)}$ we have in particular $\left[x, \mathfrak{g}^{(2)}\right]=0 \subset \mathfrak{g}^{(2)}$ for all $x \in \mathfrak{z}$. Hence $z \subset \mathfrak{g}^{(0)}$. Again we can take over the first part of the proof of [7; part III, Lemma 18] without change and get

(1) $z \subset \mathfrak{g}^{(0)}$ is an ideal of $\mathrm{g}$.

Obviously

(2) $g^{(1)} \subset z$.

(3) The Radical Conjecture holds for $g$.

Proof. We consider the solvable ideal $z \cap \mathfrak{x}$ of $g$. From (2) we know $\mathfrak{n} \subset \mathfrak{z} \cap \mathfrak{x} \subset \mathfrak{x}$. Hence we obtain that $z \cap \mathfrak{x}$ coincides either with $\mathfrak{n}$ or with $\mathfrak{x}$. In the latter case $\mathfrak{r} \subset \mathfrak{z} \subset \mathfrak{g}^{(0)}$ and $\mathfrak{g}=\mathfrak{g}^{(0)}$ follows. But we have settled this case already in 1.23 and are considering here only the case $\mathfrak{g} \neq \mathfrak{g}^{(0)}$. Therefore $\mathfrak{z} \cap \mathfrak{r}=\mathfrak{n}$. Because $\mathfrak{g}^{(1)} \subset \mathfrak{z}$ we obtain $\mathfrak{g}^{(1)} \cap \mathfrak{r} \subset \mathfrak{n}$. In particular $[j \mathfrak{n}, \mathfrak{x}] \subset \mathfrak{n}$. But then $[j n, j r]=j[j n, r]+j[n, j r]+[n, r]+k$ with some $k \in \mathfrak{f}$. It is easy to check that here all summands but $k$ are contained in $\operatorname{nil}_{0}(\mathfrak{g})$. Hence $\operatorname{ad} k=0$, whence $k=0$. Therefore $[j \mathfrak{n}, j \mathfrak{x}] \subset \mathfrak{g}^{(2)}$. Finally, $[j \mathfrak{l}, \mathfrak{l}]=j[\mathfrak{n}, \mathfrak{f}] \subset j \mathfrak{j} \subset \mathfrak{g}^{(2)}$ (where we have used once more Corollary $1, \mathrm{~d}$ ) 
of 1.4). Altogether we have shown $[j \mathfrak{n}, \mathfrak{g}] \subset \mathfrak{g}^{(2)}$. From this we obtain $\left[\mathfrak{g}^{(2)}, \mathfrak{g}\right] \subset \mathfrak{g}^{(2)}$. Hence $\mathfrak{g} \subset \mathfrak{g}^{(0)}$, a contradiction. This finishes the proof of (3) and also shows that the Radical Conjecture holds in "Case 1".

\section{§ 2. Case 2: $\mathfrak{g}=\mathfrak{g}_{0}+\mathfrak{g}_{1}$}

2.1. In this section we use $\mathfrak{g}, \mathfrak{x}, \mathfrak{n}, \mathfrak{g}^{\prime}, \mathfrak{t}$ and $\mathfrak{a}$ as introduced in 1.1. Here $t$ is a modification of a normal $j$-algebra and $a$ the modification of an abelian Kähler algebra. Let $e$ be the principal idempotent of $t$ and $\mathfrak{g}=\oplus \mathfrak{g}_{2}$ the eigenspace decomposition of $\mathfrak{g}$ relative to $\operatorname{Re}(\operatorname{ad} j e)$. In this section we consider the case where $\operatorname{Re}(\operatorname{adje})$ has only the eigenvalues 0 and 1 . We know that ad je leaves invariant $\mathfrak{t}, \mathfrak{a}, \mathfrak{x}, \mathfrak{n}$ and $\mathfrak{g}^{\prime}$. Therefore these spaces have an eigenspace decomposition as well.

2.2. In $[6 ; 4.10]$ we have seen $t_{1}=\mathfrak{n}_{1}$. Here we want to prove

LEMMA. $\mathfrak{n}_{1}=\mathfrak{r}_{1}=\mathfrak{g}_{1}$.

Procf. Clearly, $\mathfrak{g}_{1}$ is an abelian ideal of $\mathfrak{g}$. Therefore (independent of the induction) $\mathfrak{g}_{1}+j \mathfrak{g}_{1}+\mathfrak{i}$ is a Kähler algebra for which the Radical Conjecture holds. Hence (after an inessential change of $j$ ) we can assume that $g_{1}+j g_{1}$ is a solvable Kähler algebra. Let $\tilde{e}$ be the maximal idempotent of $\mathfrak{g}_{1}+j \mathfrak{g}_{1}$. Then $\tilde{e}=x_{1}+j y_{1}$ and $[j \tilde{e}, j e]=0$. But this implies $y_{1}=0$ and $\tilde{e} \in g_{1}$. Therefore $[j e, \tilde{e}]=\tilde{e}$. Hence we obtain $e=\tilde{e}$ and $\mathfrak{g}_{1}=\mathfrak{n}_{1}$ follows.

Corollary. $\mathfrak{g}_{1}+j \mathfrak{g}_{1}$ is a modification of a normal $j$-algebra with principal idempotent $e$.

2.3. Let $c$ be a minimal idempotent in $\mathfrak{g}_{1}$. Then $[j c, c]=c$ and (in the underlying normal $j$-algebra) $\left\{x \in \mathfrak{g}_{1} ;(j c, x)=x\right\}=R c$.

We consider the eigenspace decomposition of $g$ relative to $\operatorname{Re}(\operatorname{ad} j c)$, $\mathfrak{g}=\oplus_{a \in \boldsymbol{R}} \mathfrak{g}^{(a)}$. Where necessary we write $\mathfrak{g}^{(a)}=\mathfrak{g}^{(a)}(c)$. We recall that subscripts refer to weights relative to ad $j e, \mathfrak{g}_{2}^{(\alpha)}$, etc.

We know that $j c$ leaves $\mathfrak{g}_{1}, \mathfrak{g}_{0}, \mathfrak{r}, \mathfrak{n}, \mathfrak{t}, \mathfrak{a}$ and $\mathfrak{g}^{\prime}$ invariant. Hence we also have a decomposition of each of these spaces relative to ad jc.

Note that the weights of ad $j c$ in $\mathfrak{g}_{1}+j \mathfrak{g}_{1}$ and in $j \mathfrak{g}_{1}+\mathfrak{f}$ are $0, \pm 1 / 2,1$. Hence, if $a \neq 0, \pm 1 / 2,1$, then $\mathfrak{g}^{(a)} \subset \mathfrak{g}_{0}$. Moreover, by the usual argument $j \mathfrak{g}_{\lambda}^{(a)} \subset \mathfrak{g}_{\lambda}^{(a)}+\mathfrak{g}^{\prime}$.

2.4. We can use the proof of [9; Lemma 4.2] and obtain 
Lemma ([9]). Let $g \in \mathfrak{g}^{(a)}$ and $j g=\tilde{g}+x+j y+k$ where $\tilde{g} \in \mathfrak{g}^{(a)}, x, y$ $\in \mathfrak{g}_{1}$ and $k \in \mathfrak{f}$. Then $x, y \in\left(\mathfrak{g}^{(a)}+\mathfrak{g}^{(a+1)}\right) \cap \mathfrak{g}_{1}$.

2.5. In this section we prove

LEMMA. $\mathrm{g}^{(a)}=0$ if $a \notin \frac{1}{2} Z$.

Proof. Let $M=\left\{a \notin(1 / 2) Z ; \mathfrak{g}^{(a)} \neq 0\right\}$. We choose $a \in M$ so that $|a|$ is maximal. Then $\left[\mathfrak{g}^{(a)}, \mathfrak{g}_{1}\right]=0$ and in particular $\left[\mathfrak{g}^{(a)}, c\right]=0$. We will show that $\operatorname{ad}(\boldsymbol{R}+\boldsymbol{R} \boldsymbol{j} c)$ is a "symplectic representation" of $\boldsymbol{R} \boldsymbol{c}+\boldsymbol{R} j \mathrm{c}$ on $\mathfrak{g}^{(a)}$. Then, by $[8$, sect. 2,3$]$ we know that ad $j c$ has only the weights 0 , $\pm 1 / 2$ on $\mathrm{g}^{(a)}$ yielding a contradiction and proving the lemma.

$$
j \mathfrak{g}^{(a)} \subset \mathfrak{g}^{(a)}+\mathfrak{i} .
$$

This follows from Lemma 2.4. From (1) we obtain that (after an inessential change of $j$ ) we can assume $j g^{(a)} \subset g^{(a)}$ for the chosen $a \in M$. Since $|a|$ is maximal in $M$ we also have

$$
\begin{gathered}
{\left[\mathfrak{g}^{(a)}, \mathfrak{g}^{(a)}\right]=0 \quad \text { if } 2 a \notin \frac{1}{2} \boldsymbol{Z} .} \\
\rho\left(\left[\mathfrak{g}^{(a)}, \mathfrak{g}^{(a)}\right], j c\right)=0 \quad \text { if } 2 a \in \frac{1}{2} \boldsymbol{Z} .
\end{gathered}
$$

To prove (3) we note first $\mathfrak{g}^{(a)} \subset \mathfrak{g}_{0}$, since $a \in M$. Hence $\left[\mathfrak{g}^{(a)}, \mathfrak{g}^{(a)}\right] \subset \mathfrak{g}^{(2 a)}$ $\cap \mathfrak{g}_{0} \subset \tilde{\mathfrak{g}}=\oplus_{b \in(1 / 2) Z} \mathfrak{g}^{(b)}$. Since $\mathfrak{g}^{\prime} \subset \tilde{g}$, Lemma 2.4 shows $j \tilde{\mathfrak{g}} \subset \tilde{\mathfrak{g}}$. Hence $\tilde{\mathfrak{g}}$ is a Kähler subalgebra of $\mathfrak{g}$. By assumption, $\mathfrak{g}^{(a)} \neq 0, a \notin(1 / 2) Z$; therefore $\mathfrak{g} \neq \tilde{\mathfrak{g}}$. Finally, $\mathfrak{g}^{(b)}=\mathfrak{r}^{(b)}+\mathfrak{j r}^{(b)} \bmod \mathfrak{g}^{\prime}$ since $\mathfrak{g}=\mathfrak{x}+j \mathfrak{r}+\mathfrak{f}$. Hence we can apply the induction hypothesis to $\tilde{\mathrm{g}}$. Therefore $\rho\left(\tilde{\mathfrak{g}} \cap \mathfrak{g}_{0}, j c\right)=0$ and in particular $\rho\left(\left[\mathfrak{g}^{(a)}, \mathfrak{g}^{(a)}\right], j c\right)=0$. From (3) it follows immediately that ad $j c$ is symplectic on $\mathfrak{g}^{(a)}$ :

$$
\rho([j c, x], y)+\rho\left(x,[j c, y]=0 \quad \text { for all } x, y \in \mathfrak{g}^{(a)}\right.
$$

Since $\left[\mathfrak{g}^{(a)}, c\right]=0$, ad $c$ is symplectic on $\mathfrak{g}^{(a)}$ as well. Because $[j c, c]=c$, we have only left to verify (and do it by a straightforward computation)

$$
[j, \text { ad } j c-1 / 2[j, \text { ad } c]] \mathrm{g}^{(a)}=0 .
$$

This finishes the proof of the lemma.

2.6. We sharpen the last result and get

Lemma. $\quad g^{(a)}=0$ if $a \notin\{0, \pm 1 / 2, \pm 1\}$. 
Proof. Let $a \in(1 / 2) Z, a \notin\{0, \pm 1 / 2, \pm 1\}$ and suppose $\mathfrak{g}^{(a)} \neq 0$. We assume that $|a|$ is maximal. Then $\left[\mathfrak{g}^{(a)}, g_{1}\right]=0$, since ad $j c$ has only the weights $0,1 / 2,1$ in $\mathfrak{g}_{1}$. Moreover, $j \mathfrak{g}^{(a)} \subset \mathfrak{g}^{(a)}+\mathfrak{l}$ follows from Lemma 2.4 since $\mathfrak{g}^{(a)} \cap \mathfrak{g}_{1}=0$ and $\mathfrak{g}^{(a+1)} \cap \mathfrak{g}_{1}=0$. We can again assume that even $j \mathfrak{g}^{(a)} \subset \mathfrak{g}^{(a)}$ holds. From $\left[\mathfrak{g}^{(a)}, c\right]=0$ and $\left[\mathfrak{g}^{(a)}, \mathfrak{g}^{(a)}\right]=0$ we conclude that ad $c$ and ad $j c$ are symplectic maps of $\mathrm{g}^{(a)}$. As at the end of the proof of the last lemma one finishes the verification that ad is a symplectic representation of $\boldsymbol{R} c+R j c$ on $\mathfrak{g}^{(a)}$. Hence $a \in\{0, \pm 1 / 2\}$, a contradiction.

2.7. From Lemma 2.4 it is easy to derive that after an inessential change of $j$ we can assume $j \hat{g} \subset \hat{g}$ where $\hat{g}=g^{(-1)}+g^{(0)}+g^{(1)}$. Therefore $\hat{\mathfrak{g}}$ is a Kähler subalgebra of $\mathfrak{g}$. Moreover, $\mathfrak{g}^{(n)}=\mathfrak{r}^{(n)}+j \mathfrak{r}^{(n)} \bmod \left(\mathfrak{g}^{\prime} \cap \hat{g}\right)$. Hence we can apply the induction hypothesis to $\hat{g}$ in case $\hat{g} \neq g$. In this case we have $\mathrm{g}^{(-1)}=0$, whence

$$
\mathfrak{g}=\mathfrak{g}^{(-1 / 2)}+\mathfrak{g}^{(0)}+\mathfrak{g}^{(1 / 2)}+\mathfrak{g}^{(1)} \quad \text { if } \hat{\mathfrak{g}} \neq \mathfrak{g} .
$$

Before we continue to consider this case more closely we want to finish the possibility $\mathfrak{g}=\hat{\mathrm{g}}$.

\section{Lemma. If $\mathfrak{g}=\hat{\mathrm{g}}$, then the Radical Conjecture holds.}

Proof. By our assumption, $j c$ has only the weights 0 and 1 in $g_{1}$. Hence $g_{1}=g_{1}^{(0)}+g_{1}^{(0)}$ and since $c$ is a minimal idempotent $g_{1}^{(1)}=R c$. Because there is no weight $1 / 2$ we know that the underlying normal $j$-algebra $\mathfrak{g}_{1}+j \mathfrak{g}_{1}$ is the product of the subalgebras $\boldsymbol{R} c+\boldsymbol{R} j \mathrm{c}$ and $\mathfrak{g}_{1}^{(0)}+j \mathfrak{g}_{1}^{(0)}$. Since the modification derivations $D(x)$ annihilate $c$ and $j c$ we conclude that $\mathfrak{g}_{1}^{(0)}+j \mathfrak{g}_{1}^{(0)}$ is a subalgebra of the given Kähler algebra $g_{1}+j g_{1}$. We also know that $\mathfrak{l}$ leaves both algebras invariant. Set $\mathfrak{g}^{*}=\mathfrak{g}^{(-1)}+j \mathfrak{g}_{1}^{(0)}+\mathfrak{g}_{1}^{(0)}+\mathfrak{f}$. Then Lemma 2.4 shows that $g^{*}$ is $j$-invariant. It is easy to verify that $\mathrm{g}^{*}$ is a subalgebra of $\mathrm{g}$. It is straightforward to check that $\mathrm{g}^{(-1)}+\mathfrak{g}_{1}^{(0)}$ is an ideal of $\mathfrak{g}^{*}$. Clearly, $\mathfrak{g}^{(-1)}$ and $\mathfrak{g}_{1}^{(0)}$ are both abelian and since $\left[\mathfrak{g}^{(-1)}, \mathfrak{g}_{1}^{(0)}\right]$ $=\left[\mathfrak{g}_{0}^{(-1)}, \mathfrak{g}_{1}^{(0)}\right] \subset \mathfrak{g}_{1}^{(-1)}=0$ we see that $\mathfrak{g}^{(-1)}+\mathfrak{g}_{1}^{(0)}$ is an abelian ideal of the Kähler algebra $\mathfrak{g}^{*}$. Therefore the Radical Conjecture holds for $\mathfrak{g}^{*}$. Next we want to show $\mathrm{g}^{(-1)}=0$. We consider the jdempotent $e-c$ of $\mathfrak{g}_{1}^{(0)}+j \mathfrak{g}_{1}^{(0)}$. It is easy to see that the real part of adj(e-x) acts are identity map on $\mathrm{g}^{(-1)}$. From the solvable theory, applied to $\mathrm{g}^{*}$, we know that $j \mathrm{~g}^{(-1)}$ is annihilated by the real part of ad $j(e-c)$. But from Lemma 2.4 we obtain $i \mathfrak{g}^{(-1)} \subset \mathfrak{g}^{(-1)}+\mathfrak{g}_{1}^{(0)}+j \mathfrak{g}_{1}^{(0)}+\mathfrak{f}$, whence $j \mathfrak{g}^{(-1)} \subset j \mathfrak{g}_{1}^{(0)}+\mathfrak{f}$ and $\mathfrak{g}^{(-1)} \subset \mathfrak{g}_{1}^{(0)}+\mathfrak{f}$. This implies $\mathfrak{g}^{(-1)}=0$. Hence, by our assumption $\mathfrak{g}=\mathfrak{g}_{0}+\mathfrak{g}_{1}=\mathfrak{g}^{(0)}+\mathfrak{g}^{(1)}$. 
To finish our argument we consider as in [8; sect. 2.5] the space $\mathfrak{u}=$ $\{x \in \mathfrak{g} ;[x, c]=0,[j x, c]=0\} . \quad$ Clearly, $\mathfrak{i} \subset \mathfrak{u}$ and $j \mathfrak{u} \subset \mathfrak{u}$. Since $R c$ is a one dimensional ideal of $g$ we have for $x \in \mathfrak{g}:[x, c]=a c$ and $[j x, c]=b c$ for some $a, b \in \boldsymbol{R}$. A straightforward computation shows $x-a j c-b c \in \mathfrak{u}$. Hence $\mathfrak{g}=\mathfrak{u}+\boldsymbol{R} c+\boldsymbol{R} j \boldsymbol{c}$. From the definition of $\mathfrak{u}$ we derive $\mathfrak{g}_{1}^{(0)} \subset \mathfrak{u}$, whence $\mathfrak{g}_{1}^{(0)}+j \mathfrak{g}_{1}^{(0)}+\mathfrak{f} \subset \mathfrak{u}$. Next we show $[j c, \mathfrak{u}] \subset \mathfrak{u}$. Let $u \in \mathfrak{H}$, then $[[j c, u], c]=0$ and $[j[j c, u], c]=[[j c, j u]-j[c, j u]-[c, u]-k, c]=0$. Therefore $\mathfrak{u}=\mathfrak{u}^{(0)}+\mathfrak{u}^{(1)}$. Since $\mathfrak{g}^{(1)}=\boldsymbol{R} c, \quad \mathfrak{u} \subset \mathfrak{g}^{(0)}$. Let $u=u_{1}+u_{0}, \quad u_{i} \in \mathfrak{g}_{i}$. Then $u \in \mathfrak{U}$ iff $\left[u_{0}, c\right]=0$ and $\left[j u_{1}+j u_{0}, c\right]=0$. In particular $u_{0} \in \mathfrak{v}=$ $\left\{x \in \mathfrak{g}_{0} ;[x, c]=0\right\}$. On the other hand, let $v \in v$, then $[v, c]=0$ and $[j v, c]$ $=b c$. Therefore $v-b c \in \mathfrak{u}$. But $\mathfrak{u} \subset \mathfrak{g}^{(0)}$ and $v \in \mathfrak{g}_{0} \subset \mathrm{g}^{(0)}$, whence $b=0$. Therefore $\mathfrak{u}=\mathfrak{g}_{1}^{(0)}+\mathfrak{v}$. To see that $\mathfrak{u}$ is a Kähler algebra we have to show $\left[\mathfrak{b}, \mathfrak{g}_{1}^{(0)}\right] \subset \mathfrak{g}_{1}^{(0)}$. But this follows since $\mathfrak{u} \subset \mathfrak{g}^{(0)}, \mathfrak{g}^{(0)}$ is a subalgebra and $\mathfrak{g}^{(0)} \cap \mathfrak{g}_{1}=\mathfrak{g}_{1}^{(0)}$. Finally, $\mathfrak{x}_{0}=\mathfrak{x} \cap \mathfrak{g}_{0}$ acts nilpotently on $\mathfrak{g}$, therefore $\left[\mathfrak{r}_{0}, c\right]=0$ and $\mathfrak{r}_{0} \subset \mathfrak{v} \subset \mathfrak{u}$ follows. Now it is easy to see that $\mathfrak{u}=\left(\mathfrak{r}_{0}+\mathfrak{g}_{1}^{(0)}\right)$ $+j\left(\mathfrak{r}_{0}+\mathfrak{g}_{1}^{(0)}\right)+\mathfrak{l}$ holds, where $\mathfrak{r}_{0}+\mathfrak{g}_{1}^{(0)}$ is a nilpotent ideal of $\mathfrak{u}$. This implies that the Radical Conjecture holds for $\mathfrak{u}$. From this we will derive that the Radical Conjecture holds for $g$. Let $\mathfrak{h}$ be a maximal semisimple subalgebra of $\mathfrak{g}_{0}$. Then $\mathfrak{h}$ is maximal semisimple in $\mathfrak{g}$. Moreover, $[\mathfrak{h}, R c]$ $\subset R c$ implies $[\mathfrak{h}, c]=0$, i.e. $\mathfrak{h} \subset \mathfrak{b}$. Let $\mathfrak{t}_{h}$ be a maximal split solvable subalgebra of $\mathfrak{h}$. Then $t_{u}=\mathfrak{t}_{h}+\operatorname{rad}(\mathfrak{b})+\mathfrak{g}_{1}^{(0)}$ is a solvable subalgebra of $\mathfrak{u}$ and $\mathfrak{u}=\mathfrak{t}_{u}+\mathfrak{f}$ by the Radical Conjecture applied to $\mathfrak{u}$. Moreover, $\mathfrak{h} \subset \mathfrak{t}_{h}+\mathfrak{f}$. Therefore, $\mathfrak{t}_{0}=\mathfrak{t}_{h}+\operatorname{rad}\left(\mathfrak{g}_{0}\right)$ is a solvable subalgebra of $\mathfrak{g}_{0}$ and $g_{0}=t_{0}+\mathfrak{t}$ holds. Hence $t=t_{0}+g_{1}$ is a solvable subalgebra of $g$ and $\mathfrak{g}=t+\mathfrak{f}$. From this the Radical Conjecture follows.

2.8. In the last subsection we have seen that the Radical Conjecture holds if $\hat{g}(c)=\mathfrak{g}$ for some minimal idempotent $c$ of $\mathfrak{g}_{1}$. Therefore, from now on we can assume $\mathfrak{g} \neq \hat{\mathrm{g}}(c)$ for all minimal idempotents $c$ of $\mathfrak{g}_{1}$. Hence $\mathrm{g}=\mathrm{g}^{(-1 / 2)}(c)+\mathrm{g}^{(0)}(c)+\mathfrak{g}^{(1 / 2)}(c)+\mathfrak{g}^{(1)}(c)$ for all minimal idempotents $c \in \mathfrak{g}_{1}$. Applying the induction hypothesis to $\hat{g}(c)=\mathfrak{g}^{(0)}(c)+\mathfrak{g}^{(1)}(c)$ once more shows $\mathfrak{g}^{(1)}(c) \subset \mathfrak{g}_{1}$. Therefore $\mathfrak{g}_{0}^{(a)}(c) \neq 0$ implies $-1 / 2 \leq a \leq 1 / 2$. This shows that [9; Lemma 4.3] holds. This together with Lemma 2.4, (which is [9; Lemma 4.2]) enables us to carry out the rest of the arguments of [9; $\S 2]$ with few changes.

We choose minimal tripotents $c_{1}, \cdots, c_{k}$ in $\mathfrak{g}_{1}$ satisfying

$$
c_{1}+\cdots+c_{k}=e
$$




$$
\left[j c_{i}, c_{k}\right]=\delta_{i k} c_{k} .
$$

These conditions determine the $\mathrm{c}_{k}$ 's uniquely (up to permutation). For the purposes of this subsection we order $c_{1}, \cdots, c_{k}$ as in [9]. From the solvable theory (applied to $g_{1}+j g_{1}$ ) we know

$$
\left[j c_{\imath}, j c_{k}\right]=0 \quad \text { for all } i, k \text {. }
$$

Therefore we get a simultaneous eigenspace decomposition of $g$ relative to $R_{1}, \cdots, R_{l}, R_{k}=\operatorname{Re}\left(\operatorname{ad} j c_{k}\right)$. We thus get $\mathfrak{g}=\oplus \mathfrak{g}^{(1)}$ where $R_{i} x^{(1)}=\Lambda(i) x^{(1)}$ for all $x^{(1)} \in \mathfrak{g}^{(\Lambda)}$. Clearly, $\mathfrak{g}^{(\Lambda)}=\mathfrak{g}_{1}^{(1)}+\mathfrak{g}_{0}^{(\Lambda)}$.

We know $\Lambda=\Delta_{i}$ or $\Lambda=(1 / 2)\left(\Delta_{i}+\Delta_{\jmath}\right)$ on $g_{1}$ where $\Delta_{i}(k)=\delta_{i k}$. Moreover, we have seen above that $\Lambda(k)=1$ for some $k \in\{1, \cdots, l\}$ implies $\mathfrak{g}^{(\Lambda)} \subset g_{1}$ and that $\Lambda(k) \in\{0, \pm 1 / 2,1\}$ for all $k$.

As in [9] we introduce the subalgebra $\mathfrak{g}$ of $\mathfrak{g}_{0}, \mathfrak{g}=\left\{x \in \mathfrak{g}_{0} ;[x, e]=0\right\}$. Since $x-j[x, e] \in \mathfrak{g}$ for each $x \in \mathfrak{g}_{0}$ we have $\mathfrak{g}_{0}=\mathfrak{g}+j \mathfrak{g}_{1}$.

Using these fact and definitions, the proof of [9; Lemma 4.4] carries over and yields

$$
\mathrm{g}_{0}^{(\lambda)} \subset s \text { if } \Lambda \neq 1 / 2\left(\Delta_{i}-\Delta_{k}\right) \quad \text { for all } i, k .
$$

Next we consider $s \in \mathfrak{g}$ and decompose it relative to $\mathfrak{g}=\oplus \mathfrak{g}^{(1)}$. From (4) we conclude that $s^{(0)}+\sum_{a \neq b} s^{\left.(1 / 2) \Delta_{a}-1 /(2) \Delta_{b}\right)} \in \mathfrak{J}$. It is easy to see that $\left[s^{(0)}, c_{i}\right] \in \boldsymbol{R} c_{i}$ holds. Moreover, assume $0 \neq x=\left[s^{\left.(1 / 2) d_{a}-(1 / 2) d_{b}\right)}, c_{i}\right], a \neq b$, is a multiple of some $c_{k}$. Then an application of $R_{k}$ yields $1=(1 / 2)\left(\delta_{a k}-\delta_{b k}\right)$ $+\delta_{i k}$. Hence $i=k, i \neq a, b$. But then $R_{a} x=(1 / 2) x$, whence $x=0$, a contradiction. Therefore $x$ is perpendicular to $\oplus_{k=1}^{l} \boldsymbol{R} c_{k}$. Altogether $\left[s^{(0)}, e\right]=0$ follows. We thus have proven

(5) ([9]) Let $s \in \mathfrak{Z}$, then $s^{(0)} \in \mathfrak{Z}$.

Next we show

(6) ([8], [9]) Let $\Lambda=(1 / 2)\left(\Delta_{a}-\Delta_{b}\right), a \leq b$, then we have

$$
\mathfrak{g}_{0}^{(\Lambda)}=\left(\mathfrak{\xi} \cap \mathfrak{g}_{0}^{(\lambda)}\right)+\left(j \mathfrak{g}_{1}\right)^{(\Lambda)} \text {. }
$$

For $\Lambda=0$ this follows from (5) and the case $\Lambda \neq 0$ can be shown as in [8; Sect. 4.4].

We set $\sigma(x)=\operatorname{trace}\left(\operatorname{ad} x \mid \mathrm{g}_{1}\right), x \in \mathrm{g}$. The proof of [9; Lemma 4.6] carries over without change and yields $\left(y_{3}\right.$ denoting the $\xi_{\text {-component }} y$ )

(7) ([9], [8]) let $A=1 / 2\left(\Delta_{a}-\Delta_{b}\right), a \leq b$, and let $s \in \mathfrak{B} \cap g_{0}^{(1)}$. Then $\sigma\left([s, j x]_{8}\right)$ $=0$ for all $x \in \mathfrak{g}_{1}$. 
Lemma ([8]). $\operatorname{trace}\left(\operatorname{ad} s \mid g_{1}\right)=0$ for all $s \in \mathfrak{g}$.

Proof. By (5) we can assume $s \in \mathfrak{F}^{(0)}$. Using (6) and (7) we can carry out the proof of $[8$; Lemma 8 , sect. 4] without further changes and obtain the claim.

2.9. We collect the properties of $\mathfrak{g}$ which will be used in the following sections.

Lemma. a) $\mathfrak{g}=\left\{x \in \mathfrak{g}_{0} ;[x, e]=0\right\}$ is a Kähler subalgebra of $\mathfrak{g}_{0}$ and $\mathfrak{i} \subset \mathfrak{z}$.

b) $\mathfrak{g}_{0}=\mathfrak{g}+j \mathfrak{g}$ is a direct sum of vectcr spaces.

c) $\operatorname{trace}\left(\operatorname{ad} \mathfrak{g} \mid \mathfrak{g}_{1}\right)=0$

d) ad $\mathfrak{g} \mid g_{1}$ is a contained in the isotropy algebra of the homogeneous cone $K$ in $g_{1}$ which is associated with the Kähler algebra $g_{1}+j g_{1}$ and the point $e \in K$.

Proof. a) Following [8; Sec. 2, 5] we let $\mathfrak{m}=\{x \in \mathfrak{g} ;[x, e]=0$, $[j x, e]=0\}$. Clearly, $j \mathfrak{m} \subset \mathfrak{m}$. As in loc. cit. one proves $\mathfrak{g}=\mathfrak{m}+\mathfrak{g}_{1}+j \mathfrak{g}_{1}$ and $[j e, \mathfrak{m}] \subset \mathfrak{m}$. Hence $\mathfrak{m}=\mathfrak{m}_{0}+\mathfrak{m}_{1}$. But from the definition of $\mathfrak{m}$ it follows $0=\left[j m_{1}, e\right]=m_{1}$, whence $\mathfrak{m} \subset g_{0}$. Obviously, $\mathfrak{m} \subset \mathfrak{g}$. But $\mathfrak{m}$ and $\mathscr{g}$ have the same dimension since both are algebraic complements of $j \mathfrak{g}_{1}$ in g. Therefore $\mathfrak{m}=\mathfrak{g}$ and a) follows. b) and c) have been shown in the last subsection. d) This follows from [17; Proposition 4] and c).

2.10. Put $\check{\mathfrak{g}}=\left\{x \in \mathfrak{g} ;\left[x, \mathfrak{g}_{1}\right]=0\right\}$. Then $\check{\mathfrak{g}} \subset \mathfrak{g}$ is an ideal of $\mathfrak{g}$.

LEMMA. $\mathfrak{g}=\check{\mathscr{g}}+j \ddot{\mathscr{g}}+\mathfrak{l}$ and $\mathfrak{x}_{0} \subset \check{\mathfrak{g}}+j \mathfrak{g}_{1}$.

Proof. Let $r \in \mathfrak{x}_{0}=\mathfrak{x} \cap \mathfrak{g}_{0}$; then $r=s+j g_{1}, s \in \mathfrak{Z}, g_{1} \in \mathfrak{g}_{1}$. From Lemma 2.9, we know ad $g_{0} \mid g_{1} \subset$ Lie Aut $K$, the infinitesimal linear automorphisms of the cone $K$. Hence ad $\mathfrak{x}_{0} \mid \mathfrak{g}_{1}$ is an ideal in ad $g_{0} \mid g_{1}$ wbich consists of nilpotent endomorphisms. This implies ad $\mathfrak{x}_{0} \mid \mathfrak{g}_{1} \subset$ ad $j \mathfrak{g}_{1} \mid \mathfrak{g}_{1}$, whence. $\mathfrak{r}_{0} \subset \check{\mathfrak{g}}$ $+j \mathfrak{g}_{1}$. But this implies $\mathfrak{g} \subset \mathfrak{x}_{0}+j \mathfrak{x}_{0}+\mathfrak{g}_{1}+j \mathfrak{g}_{1}+\mathfrak{f} \subset \mathfrak{g}+j \mathfrak{g}+\mathfrak{l}+\mathfrak{g}_{1}+j \mathfrak{g}_{1}$ $\subset \mathfrak{g}+\mathfrak{g}_{1}+j \mathfrak{g}_{1}$ and the assertion follows.

2.11. Set $\check{\mathfrak{x}}=\left\{j g_{1} ; s+j g_{1} \in \mathfrak{r}_{0}\right.$ for some $\left.s \in \check{a}\right\}$. Denote by $\mathfrak{p}$ the Kähler subalgebra of $\mathfrak{g}_{1}+j \mathfrak{g}_{1}$ generated by $\check{x}$. Then $\mathfrak{p}=\left(\mathfrak{p} \cap \mathfrak{g}_{0}\right)+\left(\mathfrak{p} \cap \mathfrak{g}_{1}\right)$.

LemMa. a) $\check{\mathfrak{x}}$ is an ideal of $j \mathfrak{g}_{1}$.

b) $[\mathfrak{s}, \mathfrak{p}] \subset \mathfrak{s}+\mathfrak{p}$. 
c) $\mathfrak{p}+\mathfrak{z}$ is a Kähler subalgebra of $\mathfrak{g}$.

d) $\mathfrak{p}_{1}=\mathfrak{p} \cap \mathfrak{g}_{1} \neq \mathfrak{g}_{1}$.

Proof. a) Let $j b \in \check{\mathfrak{x}}$ and $s \in \breve{s}$ so that $s+j b \in r_{0}$. Then $[j a, s]+[j a, j b]$ $=[j a, s+j b] \in \mathfrak{r}_{0}$. Since $[j a, s] \in \check{\mathfrak{S}}$ and $[j a, j b] \in j g_{1}$ we conclude $[j a, j b] \in \check{\mathfrak{x}}$.

b) First we prove

(1) Let $\mathfrak{u}$ be a subalgebra of $\mathfrak{g}$ satisfying $[\mathfrak{F}, \mathfrak{u}] \subset \mathfrak{u}+\mathfrak{Z}$. Then the Lie algebra $\overline{\mathfrak{u}}$ generated by $\mathfrak{u}+j \mathfrak{u}$ satisfies $[\mathfrak{s}, \overline{\mathfrak{u}}] \subset \overline{\mathfrak{u}}+\mathfrak{g}$.

Proof. Since $\check{\xi}$ is an ideal of $g$ the condition $[\mathfrak{s}, \mathfrak{t}] \subset \mathfrak{u}+\mathfrak{g}$ is equivalent to $[j \check{\mathfrak{s}}, \mathfrak{u}] \subset \mathfrak{u}+\mathfrak{s}$ and $[\mathfrak{f}, \mathfrak{u}] \subset \mathfrak{u}+\mathfrak{s}$. But then $[j \check{s}, j u]=j[j \check{s}, u]+$ $j[\check{s}, j u]+[\check{s}, u]+k$ shows $\left[j_{\mathfrak{w}}, j \mathfrak{j}\right] \subset j \mathfrak{u}+\mathfrak{g}$ and $[k, j u]=j[k, u]+k^{\prime}$ implies $[\mathfrak{f}, j \mathfrak{u}] \subset j \mathfrak{u}+\mathfrak{s}$. Hence altogether $[\mathfrak{s}, \mathfrak{u}+j \mathfrak{u}] \subset \mathfrak{u}+j \mathfrak{u}+s . \quad$ A simple induction finishes now the proof of (1).

From (1) follows immediately

(2) Let $\mathfrak{u}$ be a subalgebra of $\mathfrak{g}$ satisfying $[\mathfrak{F}, \mathfrak{u}] \subset \mathfrak{u}+\mathfrak{z}$. Then the $j$-algebra $\tilde{\mathfrak{u}}$ generated by $\mathfrak{u}$ satisfies $[\mathcal{I}, \tilde{\mathfrak{u}}] \subset \tilde{\mathfrak{u}}+\mathfrak{\xi}$.

To prove b) it suffices now to show

(3) $[\mathfrak{z}, \check{\mathfrak{x}}] \subset \check{\mathfrak{x}}+\mathfrak{g}$.

Proof. We note that $\left[k, s+j g_{1}\right]=[k, s]+k^{\prime}+j\left[k, g_{1}\right] \in r_{0}$ if $s+j g_{1}$ $\in r_{0}$; clearly $[k, s] \in \check{\mathfrak{G}}$ if $s \in \check{\mathfrak{g}}$, whence $k^{\prime} \in \check{\mathfrak{G}}$ by Lemma 2.10. Hence $[\mathfrak{f}, \check{x}] \subset \check{x} . \quad$ Also, $\quad\left[j s^{\prime}, s+j g_{1}\right]=\left[j s^{\prime}, s\right]+\left[j s^{\prime}, j g_{1}\right]=\left[j s^{\prime}, s\right]+j\left[j s^{\prime}, g_{1}\right]+$ $j\left[s^{\prime}, j g_{1}\right]+\left[s^{\prime}, g_{1}\right]+k=\left(\left[j s^{\prime}, s\right]+j\left[s^{\prime}, j g_{1}\right]+k\right)+j\left[j s^{\prime}, g_{1}\right] \in r_{0} \cap\left(\mathfrak{s}+j g_{1}\right)$ for all $s^{\prime} \in \check{g}$ and $s, g_{1}$ as in the definition of $\check{\mathfrak{x}}$. By Lemma 2.10, we conclude $\left[j s^{\prime}, s\right]+j\left[s^{\prime}, j g_{1}\right]+k \in \check{\mathfrak{s}}$. Hence $j\left[j s^{\prime}, g_{1}\right] \in \check{\mathfrak{x}}$. This finishes the proof of (3) and thus of $b$ ).

c) Since $\mathfrak{p}$ and $\xi$ are Kähler algebras the assertion follows from $b$ ).

d) We know $\mathfrak{x} \subset \operatorname{nil}(\mathfrak{g})$. Therefore $\operatorname{ad} r$ is nilpotent for all $r \in \mathfrak{r}$. Since $\check{\mathfrak{g}}$ is an ideal of $\mathfrak{g}$ we derive from $\operatorname{ad}\left(s+j g_{1}\right)^{n} b=\left(\operatorname{ad} j g_{1} \mid g_{1}\right)^{n} b, b \in g_{1}$, that $\operatorname{ad} j g_{1} \mid g_{1}$ is nilpotent for all $j g_{1} \in \check{x}$. This implies that $\check{\mathfrak{x}}$ is perpendicular to all $j c_{i}, c_{i}$ a minimal idempotent in $g_{1}$. We order the minimal idempotents as in $[4 ;$ p. 5] and see that the last minimal idempotent $c$ is perpendicular to the clan generated by $j$ r̆. From this it follows that $\check{\mathfrak{x}}+j \check{\mathrm{x}}$ is contained in the $j$-algebra of elements of $\mathfrak{g}_{1}+j g_{1}$ which are perpendicular to $\boldsymbol{R} c+\boldsymbol{R} j \boldsymbol{c}$. Therefore $c$ is perendicular to $\mathfrak{p}_{1}$ and $\mathrm{d}$ ) follows.

2.12. We have $\mathfrak{r}_{0} \subset \mathfrak{z}+\check{\mathfrak{x}} \subset \mathfrak{g}+\mathfrak{p}$ and $\mathfrak{p}_{1}=\mathfrak{p} \cap \mathfrak{g}_{1} \subset \mathfrak{r}_{1}$. It is easy to 
verify that $\mathfrak{x}_{0}+\mathfrak{p}_{1}$ is a (solvable) ideal of $\mathfrak{p}+\mathfrak{\xi}$. Moreover, $\operatorname{dim}\left(\mathfrak{x}_{0}+\mathfrak{p}_{1}\right)$ $<\operatorname{dim} \mathfrak{r}$. Consider the Kähler subalgebra $\mathfrak{w}=\left(\mathfrak{r}_{0}+\mathfrak{p}_{1}\right)+j\left(\mathfrak{r}_{0}+\mathfrak{p}_{1}\right)+\mathfrak{f}$ of $\mathfrak{s}+\mathfrak{p}$.

Lemma. $\mathfrak{g} \subset \mathfrak{w}=\mathfrak{g}+\mathfrak{p}$.

Proof. Let $s \in \mathfrak{B}$. Then $s=r_{0}+j r_{0}^{\prime}+x_{1}+j y_{1}+k$ where $r_{0}, r_{0}^{\prime} \in \mathfrak{r}_{0}$, $x_{1}, y_{1} \in \mathfrak{g}_{1}, k \in \mathfrak{f}$. We can write $r_{0}=s_{0}+j g_{1}, r_{0}^{\prime}=s_{0}^{\prime}+j g_{1}^{\prime}$ with $s_{0}, s_{0}^{\prime} \in \check{\mathfrak{s}}$, $g_{1}, g_{1}^{\prime} \in \mathfrak{p}_{1}$. Hence $s=s_{0}+j s_{0}^{\prime}+k+j\left(g_{1}+y_{1}\right)+\left(x_{1}-g_{1}^{\prime}\right)$ and $x_{1}=g_{1}^{\prime} \in \mathfrak{p}_{1}$, $y_{1}=-g_{1} \in \mathfrak{p}_{1}$. Therefore $s \in \mathfrak{w}$. To finish the proof it suffices to show $\mathfrak{p} \subset \mathfrak{w}$. But $\mathfrak{p}=\mathfrak{p}_{0}+\mathfrak{p}_{1} \subset \mathfrak{g}_{1}+j \mathfrak{g}_{1}$ where $\mathfrak{p}_{j}=\mathfrak{p} \cap \mathfrak{g}_{j}$. Hence $\mathfrak{p}_{0}=j \mathfrak{p}_{1}$ and $\mathfrak{p} \subset \mathfrak{w}$ follows.

It is clear that we can apply the induction hypothesis to $\mathfrak{w}=\mathfrak{p}+\mathfrak{g}$.

COROLlary 1 . a) $\mathfrak{g}_{\mathfrak{S}}=\mathfrak{g}_{s}+\mathfrak{t}$ where $\mathfrak{g}_{s}$ is a solvable subalgebra of $\mathfrak{g}$.

b) After an inessential change of $j$, which does not alter $j$ on $g_{1}+j g_{1}$, we can assume $j \mathfrak{g}_{s} \subset \mathfrak{g}_{s}$.

Proof. a) Let $\mathfrak{h}$ be a maximal semisimple subalgebra of $\mathfrak{g}$ containing $[\mathfrak{f}, \mathfrak{f}]$. From the Radical Conjecture applied to $\mathfrak{w} \supset \mathfrak{g}$ it follows that a maximal compact subalgebra of $\mathfrak{h}$ is already contained in $\mathfrak{f}$. From this the claim follows.

b) follows from a) and the facts $\mathfrak{g} \cap\left(\mathfrak{g}_{1}+j \mathfrak{g}_{1}\right)=0, j \mathfrak{g} \subset \mathfrak{g}$ and $j\left(\mathfrak{g}_{1}+j \mathfrak{g}_{1}\right) \subset \mathfrak{g}_{1}+j \mathfrak{g}_{1}$.

Corollary 2. a) $\operatorname{ad}\left(\mathfrak{g}_{s}\right) \mid \mathfrak{g}_{1}$ is abelian

b) $\left[\mathfrak{g}_{s}, \mathfrak{g}_{s}\right] \subset \ddot{\mathfrak{g}}$.

Proof. Since ad $s \mid \mathfrak{g}_{1}, s \in \mathfrak{B}$, is skewadjoint relative to some inner product on $\mathfrak{g}_{1}$ we know that $\operatorname{ad}\left(\mathfrak{g}_{s}\right) \mid \mathfrak{g}_{1}$ is solvable and skewadjoint, whence abelian, proving a).

b) follows immdiately from a).

Remark. The importance of Corollary 2 is, that it deals with all of $\mathfrak{z}$ (modulo the isotropy part $\mathfrak{l}$ ).

2.13. Let $s_{0}$ be a principal idempotent of the Kähler albebra $\mathfrak{g}_{s}$ satisfying $\left[\mathfrak{f}, s_{0}\right]=0$. Since $\left[j s_{0}, s_{0}\right]=s_{0}$ we know from Corollary 2.12.2 that $s_{0} \in \mathcal{g}$ holds.

Let $D=\operatorname{Re}\left(\operatorname{ad} j s_{0}\right)$ and $\mathfrak{g}=\bigoplus_{b \in R} \mathfrak{g}^{[b]}$ be the eigenspace decomposition of $\mathfrak{g}$ relative to $D$. Then $\mathfrak{f} \subset \mathfrak{g}^{[0]}$. 
Since ad $j s_{0} \mid g_{1}$ is skewadjoint (realtive to some inner product on $\mathfrak{g}_{1}$ ) we have

(1) $\mathfrak{g}_{1} \subset \mathfrak{g}^{[0]}$.

(2) $D$ has only the eigenvalues $0, \pm 1 / 2,1$ and the eigenspaces for the eigenvalues $\pm 1 / 2,1$ are contained in $\mathfrak{F}_{s}$.

Proof. For $g \in \mathfrak{g}_{1}$ we have $\left[j s_{0}, j g\right]=j\left[j s_{0}, g\right] \bmod \mathfrak{g}$. Hence $D j g=$ $j D g \bmod \xi=0 \bmod \xi$. This implies that nonzero eigenvalues of $D$ can only occur in 3 . But $\mathfrak{f} \subset \mathfrak{g}^{[0]}$; therefore nonzero eigenvalues can only occur in $\mathfrak{F}_{s}$. Note that in $\mathfrak{F}_{s}$ only the eigenvalues $0, \pm 1 / 2,1$ can occur.

2.14. We want to apply the appendix to $D=\operatorname{Re}\left(\operatorname{adj} s_{0}\right)$. Let $\mathfrak{q}$ be the algebraic hull of ad $g \subset \operatorname{End}_{R}(\mathfrak{g})$. Then $D \in \mathfrak{q}$ is a semisimple endomorphism of $\mathfrak{g}$. Hence $D=D_{s}+D_{r}$ where $D_{s} \in \mathscr{F}$, $\mathfrak{\mathcal { C }}$ some maximal semisimple subalgebra of $\mathfrak{q}$, and $D_{r} \in \operatorname{rad} \mathfrak{q}$ satisfy $\left[D_{s}, D_{r}\right]=0$. Since $[\mathfrak{S}, \mathfrak{S}]=\mathfrak{S}$ we have $\mathfrak{S}=\operatorname{ad} \mathfrak{h}$ for some maximal semisimple subalgebra of $\mathfrak{g}$. Let $h_{0} \in \mathfrak{h}$ so that $\operatorname{ad} h_{0}=D_{s}$. Since $\operatorname{ad} h_{0}$ is semisimple with only real eigenvalues, $h_{0}$ is contained in some Cartan subalgebra of $\mathfrak{h}$. Therefore, if ad $h_{0}$ has an eigenvalue $\lambda \neq 0$, then it also has the eigenvalue $-\lambda \neq 0$. Moreover, there exist $x \in \mathfrak{h}_{\lambda}$ and $y \in \mathfrak{h}_{-\lambda}$ such that $[x, y]$ acts semisimply on $g$. Since the eigenvalues of ad $h_{0}$ are also eigenvalues for $D$ we conclude $\lambda= \pm 1 / 2$ But we have seen in 2.13 that the eigenvalues $\pm 1 / 2$ of $D$ are only attained in the solvable Lie aglebra $\mathfrak{g}_{s}$. Hence $[x, y]$ is nilpotent on $\mathfrak{g}$, a contradiction. This shows

LEMMA. $\mathfrak{h} \subset \mathfrak{g}^{[0]}$.

2.15. We consider the subalgebra $\mathfrak{m}=\mathfrak{g}^{[0]}+\mathfrak{g}^{[1]}$ of $\mathfrak{g}$.

Lemma. a) $\mathfrak{m}$ is a Kähler subalgebra of $\mathfrak{g}$

b) $\mathfrak{m}=(\mathfrak{x} \cap \mathfrak{m})+j(\mathfrak{x} \cap \mathfrak{m})+\mathfrak{x}$.

Proof. From 2.10 we know that $\check{\xi}$ is an ideal of $\mathrm{g}$. We have seen above $\mathfrak{g}^{[\lambda]} \subset \mathcal{g}$ if $\lambda \neq 0$. Replacing $e$ by $\mathfrak{g}_{0}, \mathfrak{n}$ by $\ddot{\mathfrak{s}}, \hat{\mathfrak{n}}$ by $\breve{s}^{[1 / 2]}$ and defining $\mathfrak{q}=\left\{x \in \mathfrak{g} ;\left[x, s_{0}\right] \in \hat{\mathfrak{n}},\left[j x, s_{0}\right] \in \hat{\mathfrak{n}}\right\}$ it is straightforward to check that with the exception of 4.17 the results of 4.13 through 4.22 , of [6] still hold. It is easy to verify that the proofs of 4.25 and 4.20 of [6] can be applied in our situation as well and we obtain the assertion.

2.16.

Lemma. If $\mathrm{g}^{[0]}+\mathrm{g}^{[1]} \neq \mathrm{g}$, then then Radical Conjecture holds. 
Proof. By our assumption and Lemma 2.15, we can apply the induction hypothesis to $\mathfrak{g}^{[0]}+\mathfrak{g}^{[1]}$. Therefore, there exists a solvable subalgebra $\mathfrak{g}^{[0]}$ so that $\mathfrak{g}^{[0]}+\mathfrak{l}=\mathfrak{g}^{[0]}$ holds. But then $\mathfrak{q}=\mathfrak{g}^{[0]}+\mathrm{rad} \mathfrak{g}$ is a solvable subalgebra of $\mathfrak{g}$ satisfying $\mathfrak{q}+\mathfrak{t}=\mathfrak{g}$. Since $\mathfrak{r} \subset \operatorname{nil}(\mathfrak{g})$ we have $\mathfrak{r} \subset \mathfrak{q}$. Hence, after an inessential change of $j$ we see that $\mathfrak{x}+j \mathfrak{x}$ is a solvable Kähler subalgebra of $\mathfrak{g}$ and the assertion follows.

2.17. It is clear that we have only to consider the case $\mathfrak{g}=\mathfrak{g}^{[0]}+\mathfrak{g}^{[1]}$. Here we assume - in addition to our previous assumptions - that $\mathfrak{n}$ is chosen so that the rank of the maximal idempotent $e$ of $\mathrm{g}^{\prime}$ is maximal.

Lemma. If $\mathrm{g}=\mathrm{g}^{[0]}+\mathrm{g}^{[1]}$, then the Radical Conjecture holds.

Proof. (1) We can assume that $\mathfrak{g}^{[1]}+j \mathfrak{g}^{[1]}$ is a solvable Kähler algebra with principal idempotent $s_{0}$.

(2) Let $\mathfrak{u}=\left\{x \in \mathfrak{g} ;\left[x, s_{0}\right]=0,\left[j x, s_{0}\right]=0\right\}$. Then $j \mathfrak{u} \subset \mathfrak{u}$ and as in [8; sect. 2.5] one proves $\mathfrak{g}=j^{[1]}+\mathfrak{g}^{[1]}+\mathfrak{u}$ and $\left[j s_{0}, \mathfrak{t}\right] \subset \mathfrak{u}$. Then $\mathfrak{u}=$ $\mathfrak{u}^{[0]}+\mathfrak{u}^{[1]}$ and $0=\left[j \mathfrak{u}^{[1]}, s_{0}\right]=\mathfrak{u}^{[1]}$. Hence $\mathfrak{g}^{[0]}=j \mathfrak{g}^{[1]}+\mathfrak{u}$.

(3) $g^{[1]} \subset r \cap s ̌$ : We know $s_{0}=x+j y+a+j b+k$ where $x, y \in \mathfrak{r}^{[1]}$, $a, b \in \mathfrak{r}^{[0]}$ and $k \in \mathfrak{f}$. We split $b=j d+u, d \in \mathfrak{g}^{[1]}, u \in \mathfrak{u}$. Then $s_{0}=x-d$ and $\left[b, s_{0}\right]=\left[j d+u, s_{0}\right]=d \in \mathfrak{x}^{[1]}$. Therefore $s_{0} \in \mathfrak{x}^{[1]}$, whence $\mathfrak{g}^{[1]}=\left[j \mathrm{~g}^{[1]}, s_{0}\right]$ $\subset \mathfrak{r}^{[1]} \cap \breve{s}$.

(4) From (3) and Lemma 2.2, we know $g_{1}+\mathfrak{g}^{[1]} \subset \mathfrak{x}$. We consider $A=\mathfrak{g}_{1}+\mathfrak{g}^{[1]}+[\mathfrak{r}, \mathfrak{x}]=\mathfrak{g}_{1}+\mathfrak{g}^{[1]}+\left[\mathfrak{r}^{[0]}, \mathfrak{r}^{[0]}\right]+\mathfrak{g}_{1}+\mathfrak{g}^{[1]}+\left[\mathfrak{r}_{0}^{[0]}, \mathfrak{r}_{0}^{[0]}\right]$. If $A=\mathfrak{r}$, then $\mathfrak{g}_{1}+\left[\mathfrak{x}_{0}^{[0]}, \mathfrak{x}_{0}^{[0]}\right]=\mathfrak{x}^{[0]}$ and $\mathfrak{x}_{0}^{[0]}=0$ follows. But then $\mathfrak{x}=\mathfrak{g}_{1}+\mathfrak{g}^{[1]}$ is, by (3), an abelian ideal of $g$ and the Radical Conjecture follows. If $A \neq \mathfrak{r}$, then we can find an ideal $\tilde{\mathfrak{n}}$ of $g$ satisfying $A \subset \tilde{\mathfrak{n}} \varsubsetneqq \mathfrak{x}$. But then the rank of a maximal idempotent $\tilde{e}$ associated with $\tilde{\mathfrak{n}}$ is greater than the rank of $e$ if $\mathrm{g}^{[1]} \neq 0$. This would be a contradiction to our choice of $\mathfrak{n}$. Therefore $\mathrm{g}^{[1]}=0$. This implies $\mathfrak{g}_{0}=0$ and $\mathfrak{g}_{s}$ is a modification of an abelian Kähler algebra. The rest of this proof is a simplification of a previous version. The preser.t version is due to $K$. Nakajima. We consider $\operatorname{rad}_{n}(\breve{\mathscr{g}})=\{x \in \operatorname{rad}(\breve{\mathscr{C}}) ; \operatorname{ad} x \mid \breve{g}$ is nilpotent $\}$

(5) $\mathfrak{r}_{0} \subset \operatorname{rad}_{n}(\breve{\mathfrak{G}})+\left[j g_{1}, j g_{1}\right]:$ Let $x \in \mathfrak{r}_{0}$. Then $x=s+z$ where $s \in \check{\mathscr{g}}$ and $z \in\left[j g_{1}, j g_{1}\right]$ by Lemma 2.10. Note that $B=\mathfrak{x}_{0}+\left[j g_{1}, j g_{1}\right]$ is a solvable subalgebra of $g_{0}$ and that $x$ and $z$ are contained in the ideal $B_{n}=$ $\{x \in B ; \operatorname{ad} x \mid \mathrm{g}$ is nilpotent $\}$ of $B$. Hence $s \in B_{n}$. Let $\mathfrak{h}$ be a maximal semisimple subalgebra of $\check{\mathfrak{G}}$ and decompose $s=s^{\prime}+s^{\prime \prime}$, where $s \in \operatorname{rad}(\check{\mathfrak{G}})$, 
$s^{\prime \prime} \in \mathfrak{h}$. Then $\operatorname{ad} s^{\prime \prime} \mid \mathfrak{h}$ is nilpotent since $s \in B_{n}$. But since $\mathrm{g}^{[1]}=0$ we know that $\mathfrak{g}$ corresponds to a flat homogeneous Kähler manifold. Therefore $\mathfrak{h}$ is a compact semisimple Lie algebra. Hence ad $s^{\prime \prime} \mid \mathfrak{h}=0$, whence $s^{\prime \prime}=0$, $s=s^{\prime}$ and (5) follows.

(6) $\operatorname{rad}_{n}(\mathfrak{G})$ is an abelian ideal of $\mathfrak{g}$ : Since $\ddot{\mathfrak{g}}$ is an ideal of $\mathfrak{g}$ we know that $\operatorname{rad}(\breve{\mathfrak{g}})$ is ideal of $\mathfrak{g}$ and $[\mathfrak{g}, \operatorname{rad}(\breve{\mathfrak{G}})] \subset \operatorname{nil}_{0}(\mathfrak{\mathfrak { g }}) \subset \operatorname{rad}_{n}(\check{\mathfrak{G}})$ follows by $[2 ; \S 5$, Proposition 6$]$, where $\operatorname{nil}_{0}(\breve{g})$ denotes the maximal nilpotent ideal of $\check{\mathfrak{g}}$. Therefore $\operatorname{rad}_{n}(\mathfrak{g})$ is an ideal of $\mathrm{g}$. Moreover, since $\breve{\mathfrak{G}}$ corresponds to a flat homogeneous Kähler manifold, $\operatorname{rad}_{n}(\check{\mathscr{G}})$ is abelian.

From (6) we obtain

(7) $\operatorname{rad}_{n}(\mathfrak{\mathfrak { G }})+\mathfrak{g}_{1}$ is an abelian ideal of $\mathfrak{g}$. To prove that the Radical Conjecture holds for $\mathfrak{g}$ it suffices now to note that $\mathfrak{g}=\left(\operatorname{rad}_{n}(\check{\mathfrak{g}})+\mathfrak{g}_{1}\right)+$ $j\left(\operatorname{rad}_{n}(\breve{g})+\mathfrak{g}_{1}\right)+\mathfrak{l}$ holds.

This finishes the proof of "Case 2".

§3. Case 3. $\mathfrak{g}=\mathfrak{g}_{-1 / 2}+\mathfrak{g}_{0}+\mathfrak{g}_{1 / 2}+\mathfrak{g}_{1}$

3.1. We use the notation of [6] as before (see 2.1). Since -1 is not a weight for adje, we have from [6; Lemma 4.19]

$$
\rho(e, q)=0 \text {. }
$$

Then Lemma 4.21 of [6] simplifies to

$$
\rho\left(e^{t \operatorname{ad} j e} u, e^{t \text { adje}} v\right)=e^{t} \rho\left(j e,[u, v]_{1}^{\prime}\right)+\text { const. }
$$

for all $u, v \in \mathfrak{g}, t \in \boldsymbol{R}$. In particular, we have

$$
\rho\left(\mathfrak{g}_{\lambda}, \mathfrak{g}_{\mu}\right)=0 \quad \text { if } \lambda+\mu \neq 0,1 .
$$

We also recall from [6; Lemma 4.26] that the Radical Conjecture holds for the Kähler subalgebra $\mathfrak{g}_{0}+\mathfrak{g}_{1}$ of $\mathfrak{g}$. Note $\mathfrak{i} \subset \mathfrak{g}_{0}$. Moreover, we have $\mathfrak{g}_{0}=\mathfrak{w}_{0}+\mathfrak{t}_{0}+j \mathfrak{g}_{1}+\mathfrak{t}$ where $\mathfrak{w}_{0}+\mathfrak{t}_{0}$ is a modification of a split solvable Kähler algebra and $\mathfrak{w}_{0}+t_{0}+j \mathfrak{g}_{1}+\mathfrak{g}$ is a solvable Kähler subalgebra of $\mathfrak{g}_{0}+\mathfrak{g}_{1}$.

As in Lemma 2.2 one proves

$$
\mathfrak{g}_{1}=\mathfrak{n}_{1}=\mathfrak{r}_{1} .
$$

3.2. We consider the subspace $\mathfrak{w}=\mathfrak{g}_{1 / 2}+\mathfrak{g}_{-1 / 2}+\mathfrak{f}$ of $\mathfrak{g}$. Then $\mathfrak{j w} \subset \mathfrak{w}$ since $j g_{2} \subset g_{2}+g^{\prime}$ and $\rho\left(\mathfrak{w}, g_{0}+g_{1}\right)=0$ by (3.3). Therefore, after an inessential change of $j$, we can even assume $j\left(\mathfrak{g}_{1 / 2}+\mathfrak{g}_{-1 / 2}\right) \subset \mathfrak{g}_{1 / 2}+\mathfrak{g}_{-1 / 2}$. 
3.3. Let $c$ be the vector space of real parts of adjc, $c \in \mathfrak{g}_{1}+\mathfrak{t}_{0},[j c, c]$ $=c$. Let $\mathfrak{c} \subset \mathfrak{b} \subset$ End $g$ be a maximal abelian subalgebra of the algebraic hull $\overline{\operatorname{adg}}$ which consists of semisimple endomorphisms. Since $\operatorname{Re}(\operatorname{adje}) \in \mathfrak{c}$ it follows $\mathfrak{b} \subset \overline{\mathrm{adg}_{0}}$. From the appendix it follows that there exists a maximal semisimple subalgebra $\mathfrak{h}$ of $\mathfrak{g}$ and an abelian subalgebra $\mathfrak{A} \subset$ $\operatorname{rad} \overline{\operatorname{adg}}$ such that $\mathfrak{b} \subset$ ad $\mathfrak{h}+\mathfrak{U}$ and $\mathfrak{U} \mathfrak{h}=0$. The maximality of $\mathfrak{b}$ implies $\mathfrak{b}=(\mathfrak{b} \cap \operatorname{ad} \mathfrak{h})+\mathfrak{A}$, where $\mathfrak{b} \cap$ ad $\mathfrak{h}$ is a Cartan algebra of ad $\mathfrak{h} \cong \mathfrak{h}$. In particular $R=\operatorname{Re}$ adje $=\operatorname{ad} h_{0}+R_{r}$ where $R_{r} \mathfrak{h}=0$ and $h_{0} \in \mathfrak{h}$. Hence the eigenvalues of $\operatorname{ad} h_{0}$ in $\mathfrak{h}$ are also eigenvalues of $R$ in $\mathfrak{g}$. Moreover, if $\lambda \neq 0$ is an eigenvalue of $\mathrm{ad} h_{0}$ in $\mathfrak{h}$, then also $-\lambda$ is an eigenvalue of $\operatorname{ad} h_{0}$ in $\mathfrak{h}$. Since $R$ has only the eigenvalues $0, \pm 1 / 2$, this implies $\lambda= \pm 1 / 2$. If ad $h_{0}$ has only the eigenvalue 0 in $\mathfrak{h}$, then the Radical Conjecture follows by the argument of [6; Lemma 4.32].

Hence from now on we assume that ad $h_{0}$ has a nonzero eigenvalue in $\mathfrak{h}$.

Then $\mathfrak{h}=\mathfrak{h}_{-1 / 2}+\mathfrak{h}_{0}+\mathfrak{h}_{1 / 2}$ and $\mathfrak{h}_{\lambda} \neq 0$ for all $\lambda$. (We will show in the rest of this paper that this assumption leads to a contradiction). Moreover, we can assume that $\mathfrak{w}_{0}+t_{0}+j g_{1}$ contains a maximal split solvable subalgebra of $\mathfrak{h}_{0}$ and that it also contains the Cartan algebra of $\mathfrak{h}$ which corresponds to $\mathfrak{b} \cap$ ad $\mathfrak{h}$.

Let $(\cdot, \cdot)$ denote the product in the unmodified algebra underlying $\mathfrak{g}_{0}+\mathfrak{g}_{1}$ and denote by ãd its adjoint representation.

Then $\operatorname{Re}(\operatorname{adj} c)=$ ãdjc in $\mathfrak{g}_{0}+\mathfrak{g}_{1}$ for all minimal idempotents $c$ of $g_{1}+t_{0}$.

Lemma. Let $x \in \mathfrak{g}_{0}$ such that $\operatorname{ad} x \in \mathfrak{b}$ holds. Then there exists a linear combination $y$ of idempotents of $\mathfrak{g}_{1}+\mathfrak{t}_{0}$ and $u \in \mathfrak{w}_{0}$ such that $x=j y+u$ and

a) $\left[x, \mathfrak{w}_{0}\right] \subset \mathfrak{w}_{0}$,

b) $[x, u]=0,[x, j u]=0,[j u, u]=0$,

c) $(j c, j]=0$ for all idempotents $c \in \mathfrak{g}_{1}+\mathfrak{t}_{0}$,

d) $[\operatorname{ad} x, \operatorname{Re}(\operatorname{adjc})]=0$ for all idempotents $c \in \mathfrak{g}_{1}+\mathfrak{t}_{0}$,

Proof. Let $x \in \mathfrak{g}_{0}$ and $\operatorname{ad} x \in \mathfrak{b}$, then ad $x$ lies in the span of all $\operatorname{Re}(\operatorname{adjc}), c$ a minimal idempotent of $g_{1}+t_{0}$. This implies $x=j y+u$ where $y$ is a linear combination of minimal idempotents and $u \in \mathfrak{g}_{0}+\mathfrak{g}_{1}$ such that $(j c, u)=0$ for all idempotents $c$. Moreover, we can assume that $u$ is perpendicular to all $j c$. Then $u \in \mathfrak{w}_{0}$. Since the modification 
derivations $D(v)$ of $\mathfrak{g}_{0}+\mathfrak{g}_{1}$ annihilate all idempotents we already get a). We also note that $\mathrm{c}$ ) and $\mathrm{d}$ ) are clear as well. In particular $(x, u)=0$. To see that also $[x, u]=0$ holds we note $D(x)=0$, since ad $x \in \mathfrak{b}$, whence $[x, u]=(x, u)-D(u) x=-D(u) u$. Since ad $x$ is selfadjoint and $D(u)$ skew adjoint relative to the inner product $\rho(a, j b)$ on $\mathfrak{w}_{0}$ we obtain $D(u) u=0$ and $[x, u]=0$. Let $\mathfrak{w}_{0}=\hat{\mathfrak{w}}_{0}+\hat{\mathfrak{w}}_{1}$ as in [5;3.3]. Then adjc leaves $\hat{\mathfrak{w}}_{0}$ and $\hat{\mathfrak{w}}_{1}$ invariant. Hence $\left[x, u_{1}\right]=0$ and $\left(j c, u_{i}\right)=0$ where $u=u_{0}+u_{1}, u_{i} \in \hat{\mathfrak{w}}_{i}$. But then $\left(j c, j u_{i}\right)=0$ and $\left[x, j u_{0}\right]=\left(x, j u_{0}\right)=0$ follows. Finally, $\left[x, j u_{1}\right]=$ $\left(x, j u_{1}\right)-D\left(j u_{1}, x\right)=-D\left(j u_{1}\right) u_{0} . \quad$ But $[x, \hat{\mathfrak{w}}] \subset \hat{\mathfrak{w}}_{1}$ implies $\left[x, w_{1}\right]=\left(x, w_{1}\right)-$ $D\left(w_{1}\right) x=\left(j y, w_{1}\right)+\left(u, w_{1}\right)-D\left(w_{1}\right) u \in \hat{\mathfrak{w}}_{1}$ for all $w_{1} \in \hat{\mathfrak{w}}_{1}$. Since $\left(j y, w_{1}\right) \in \hat{\mathfrak{w}}_{1}$, $\left(u, w_{1}\right)=0$ and $D\left(w_{1}\right) u \in \hat{\mathfrak{w}}_{0}$ we obtain $D\left(w_{1}\right) u=0$. From this we derive $\left[x, j u_{1}\right]=0$ and $[j u, u]=0$.

Remark. In what follows we will use frequently the representation theory of $\operatorname{sl}(2, \boldsymbol{R})$. We will only consider such copies of $\operatorname{sl}(2, \boldsymbol{R})$ which are of type $\operatorname{sl}(2, \boldsymbol{R}) \cong \boldsymbol{R} f_{-1 / 2}+\boldsymbol{R} f_{0}+\boldsymbol{R} f_{1 / 2}, \quad$ ad $f_{0} \in \mathfrak{b} \cap$ ad $\mathfrak{h}$ and $f_{0} \in \mathfrak{w}_{0}+\mathfrak{t}_{0}$ $+j g$.

It is clear that we can apply the above lemma to $f_{0}$.

We would like to point out that we can actually find $f_{\lambda} \in g_{\lambda}, \lambda= \pm 1 / 2$, 0 , so that in addition to the above properties $f_{\lambda}$ is a simultaneous eigenvector for all $b \in \mathfrak{b}$.

We will make it explicitly clear where we use $f_{2}$ 's with this additional property. The other properties will always tacitely be assumed.

3.4. In this section we consider the action of $\operatorname{sl}(2, \boldsymbol{R}) \cong \boldsymbol{R} f_{-1 / 2}+\boldsymbol{R} f_{0}$ $+\boldsymbol{R} f_{1 / 2}$ on $\mathrm{g}$. We know that ad $f_{0}$ has only integral eigenvalues and in an irreducible representation all integers $m, m-2, \cdots,-m$ occur. Moreover, starting from an appropriately chosen eigenvector $x_{1}$ in $g_{1}$ we get a basis of an irreducible representation of $\operatorname{sl}(2, R)$ in $\mathfrak{g}$ by applying $\operatorname{ad} f_{-1 / 2}$ to $x_{1}$. The eigenvalues of ad $f_{0}$ in $g_{1}$ are therefore all non-negative or all non-positive (depending on the sign in $\left[f_{0}, f_{-1 / 2}\right]= \pm 2 f_{-1 / 2}$ ) and only the integers $0,1,2,3$ can occur (for simplicity we assume that only non-negative integers occur in $g_{1}$; the other case follows by the same arguments). Thus we get the following chart indicating the chains of eigenvalues that can possibly occur in some irreducible representation of $\operatorname{sl}(2, \boldsymbol{R})$ in $\mathrm{g}$. Note that the vector space corresponding to the various integers in the same row all have the same dimension. 


\begin{tabular}{cccc}
\hline $\mathfrak{g}_{-1 / 2}$ & $\mathfrak{g}_{0}$ & $\mathfrak{g}_{1 / 2}$ & $\mathfrak{g}_{1}$ \\
\hline-3 & -1 & 1 & 3 \\
& -2 & 0 & 2 \\
& & -1 & 1 \\
-2 & 0 & 2 & 0 \\
& -1 & 1 & \\
-1 & 1 & 0 & \\
& 0 & & \\
\hline
\end{tabular}

3.5. We write $f_{0}=j d+t_{0}+w_{0}$ where $d \in \mathfrak{g}_{1}, t_{0} \in \mathfrak{t}_{0}, w_{0} \in \mathfrak{w}_{0}$. We know that $d$ is a linear combination of idempotents, $d=3 d_{3}+2 d_{2}+d_{1}$, $\left[j d_{\lambda}, d_{\lambda}\right]=d_{\lambda}, \lambda=1,2,3$. We set $d_{0}=e-d_{1}-d_{2}-d_{3}$. Here some of the $d_{\lambda}$ may be 0 . In what follows we use the algebra $\mathscr{A}$ on $g_{1}$ associated with $e \in \mathfrak{g}_{1}$ and the tube domain $\mathfrak{g}_{1}+j \mathfrak{g}_{1}$ in [4].

Lemma. $\mathscr{A}=\mathscr{A}_{1}\left(d_{3}+d_{1}\right) \oplus \mathscr{A}_{1}\left(d_{2}+d_{0}\right)$ as product of algebras.

Proof. (1) $d_{3} \neq 0, d_{2} \neq 0$ implies $\mathscr{A}_{1 / 2}\left(d_{3}, d_{2}\right)=\left\{x \in \mathscr{A} ;\left(j d_{3}, x\right)=(1 / 2) x\right.$ $\left.=\left(j d_{2}, x\right)\right\}=0$ since $\left[f_{0}, x\right]=\left(f_{0}, x\right)=3\left(j d_{3} \cdot x\right)+2\left(j d_{2}, x\right)=(5 / 2) x$ and $5 / 2$ is not an eigenvalue for ad $f_{0}$. Similarly one proves

(2) $\mathscr{A}_{1 / 2}\left(d_{2}, d_{1}\right)=0, \mathscr{A}_{1 / 2}\left(d_{1}, d_{0}\right)=0, \mathscr{A}_{1 / 2}\left(d_{3}, d_{0}\right)=0$.

From (1) and (2) we get the claim.

3.6. We will need some information on the eigenvalues of $\operatorname{adj} d_{i}$.

Lemma. Let $c \in \mathfrak{g}_{1}$ satisfy $[j c, c]=c$ and $[\mathfrak{f}, c]=0$. Then adjc has only the weights $0, \pm 1 / 2,1$ or the weights $0,+1$ in $\mathrm{g}$.

Proof. Let $x \in \mathfrak{g}$. Then $x=x^{\prime}+q$ where $x^{\prime} \in \mathfrak{g}^{\prime}$ and $q \in \mathfrak{q}$. Since $\mathfrak{g}_{1} \subset \mathrm{g}^{\prime}$ we can assume $q=q_{0}+q_{1 / 2}+q_{-1 / 2}$ where $q_{\lambda} \in \mathfrak{q} \cap \mathfrak{g}_{\lambda}$. We also write $x^{\prime}=\sum x_{i}^{\prime}$.

$$
\rho\left(j c, e^{t \text { ad } j c} x\right)=\rho\left(j c, e^{t \text { ad } j c} x_{1}^{\prime}\right) .
$$

Proof. From [6; Corollary 4.22] we know $\rho\left(j c, \mathfrak{g}_{1 / 2}+\mathfrak{g}_{-1 / 2}\right)=0$. We also have $\rho\left(j c, g_{0}\right)=0$. Hence $\rho\left(j c, W x_{1}^{\prime}+W x_{1 / 2}^{\prime}+W x_{-1 / 2}^{\prime}+W x_{0}^{\prime}\right)=\rho\left(j c, W x_{1}^{\prime}\right)$ where $W=e^{t \text { ad } j c}$.

Decomposing $x_{1}^{\prime}$ further into weight vectors of adjc we get $x_{1}^{\prime}=y^{[0]}$ 
$y^{[1 / 2]}+y^{[1]}$ and

$$
\rho\left(j c, e^{t \text { ad } j c} x\right)=e^{t} \rho\left(j c, y^{[1]}\right) .
$$

From this we derive, using [7; chap. III, Lemma 9]

$$
\rho\left(e^{t \text { ad } j c} u, e^{t \text { ad } j c} v\right)=a e^{t}+b .
$$

In particular, we obtain from this for the weight spaces $\mathrm{g}^{[\lambda]}$ of $L=\operatorname{Re}(\operatorname{adjc})$ in $g$ :

$$
\rho\left(\mathfrak{g}^{[\lambda]}, \mathfrak{g}^{[\mu]}\right)=0 \quad \text { if } \lambda+\mu \neq 0,1 .
$$

Next we prove (that after an inessential change of $j$ )

$$
j \mathfrak{g}^{[\lambda]} \subset \mathfrak{g}^{[\lambda]} \quad \text { if } \lambda \neq 0, \pm 1 / 2, \pm 1,3 / 2 .
$$

Proof. From the integrability condition and $\mathfrak{g}_{1}=\mathfrak{n}_{1}$ we get as usual $j \mathrm{~g}^{[\lambda]} \subset \mathrm{g}^{[\lambda]}+\mathrm{g}^{\prime}$. Hence for $x \in \mathrm{g}^{[\lambda]}$ we have $j x=y+z$ where $y \in \mathfrak{g}^{[\lambda]}$ and $z \subseteq \mathrm{g}^{\prime}$. We note that $\lambda+\{0, \pm 1 / 2,1\} \in\{0,1\}$ implies $\lambda \in\{0, \pm 1, \pm 1 / 2,3 / 2\}$. But we have excluded these values for $\lambda$, whence $\rho\left(\mathfrak{g}^{[\lambda]}, \mathfrak{g}^{\prime}\right)=0$. In particular $0=\rho(x, z)=\rho(j x, j z)=\rho(y+z, j z)=\rho(z, j z)$. Hence $z \in \mathfrak{l}$. But then $j \mathrm{~g}^{[\lambda]} \subset \mathrm{g}^{[\lambda]}+\mathfrak{t}$ and the assertion follows.

Since $2 \lambda \neq 0,1$ if $\lambda \neq 0, \pm 1 / 2, \pm 1,3 / 2$ and $k \subset \mathrm{g}^{[0]}$ we obtain from (5):

$$
\mathfrak{g}^{[\lambda]}=0 \quad \text { if } \lambda \neq 0, \pm 1 / 2, \pm 1,3 / 2 \text {. }
$$

Using (4) we prove as in [6; Lemma 4.25]

$$
j \mathfrak{g}^{[n]} \subset \mathfrak{g}^{[n]}+\mathfrak{g}^{[[1]}+\mathfrak{g}^{\prime[0]} \quad \text { for all } n \in Z \text {. }
$$

Now we can repeat the proof of [6; Lemma 4.30] and obtain

$$
\mathrm{g}^{[3 / 2]}=0 .
$$

Finally, the argument of [6; Lemma 4.26] is applicable in our situation and yields

$$
\begin{aligned}
& \hat{\mathfrak{g}}=\mathfrak{g}^{[-1]}+\mathfrak{g}^{[0]}+\mathfrak{g}^{[1]} \text { is a } j \text {-invariant subalgebra and } \\
& \hat{\mathfrak{g}}=(\mathfrak{x} \cap \mathfrak{g})+j(\mathfrak{x} \cap \mathfrak{g})+\mathfrak{f} \text {. }
\end{aligned}
$$

We consider the two possibilities $\hat{\mathrm{g}}=\mathfrak{g}$ or $\hat{\mathrm{g}} \neq \mathfrak{g}$. In the latter case we can apply the induction hypothesis and obtain $\mathrm{g}^{[-1]}=0$ (and from this the assertion). If $\hat{\mathrm{g}}=\mathfrak{g}$, then we have again two subcases. The first, $\mathrm{g}^{[-1]}=0$, is exactly what we want. The second case, $\mathrm{g}^{[-1]} \neq 0$, allows us to argue as in [6; Lemma 4.32] so that the Radical Conjecture holds in 
this case. But then $j c$ does not have the eigenvalue -1 in $\mathfrak{g}$, so that this case actually does not occur. This proves the claim.

3.7. By the result of the last section we can assume that $\operatorname{adj}\left(d_{3}+d_{1}\right)$ and $\operatorname{adj}\left(d_{2}+d_{0}\right)$ have only the (real) eigenvalues $0, \pm 1 / 2,1$ in g. Moreover, these weights occur in the eigenspaces of ad $f_{0}$ in the spaces $\mathfrak{g}_{\lambda}$. In the proof of the last section we have also seen that for $g^{[0]}+g^{[1]}$ the Radical Conjecture holds, where $\mathrm{g}^{[1]}$ is defined for $j c$ as in 3.6. We can assume $\mathrm{g}^{[1]}+\mathrm{g}^{[-1 / 2]} \neq 0$.

The following argument is a simplification of a previous version of the proof. We use ideas of K. Nikajima.

First, it is easy to see that $j g_{1 / 2}$ is invariant under $j e$. Hence $j g_{1 / 2}=$ $\left(j g_{1 / 2}\right) \cap \mathfrak{g}_{1 / 2}+\left(j g_{1 / 2}\right) \cap \mathfrak{g}_{-1 / 2}$ and $\mathfrak{g}_{1 / 2}=\mathfrak{H}_{1 / 2}+\mathfrak{w}_{1 / 2}$, where $\mathfrak{u}_{1 / 2}=\left\{x \in \mathfrak{g}_{1 / 2}, j x \in \mathfrak{g}_{-1 / 2}\right\}$ and $\mathfrak{w}_{1 / 2}=\left\{x \in \mathfrak{g}_{1 / 2}, j x \in \mathfrak{g}_{1 / 2}\right\}$.

A direct computation shows that $\mathfrak{w}_{1 / 2}$ is invariant under $j \mathfrak{g}_{1}$. Therefore $j \mathfrak{g}_{1}+\mathfrak{w}_{1 / 2}+\mathfrak{g}_{1}$ is a Kähler algebra of domain type. In particular for $c_{1}=d_{3}+d_{1}$ and $c_{2}=d_{2}+d_{0}$ we know that $j c_{1}, j c_{2}$ have only the eigenvalues 0 or $1 / 2$ on $\mathfrak{w}_{1 / 2}$ and the sum of their eigenvalues adds up to $1 / 2$.

Next we consider $\mathfrak{u}_{1 / 2}$. We know $j \mathfrak{u}_{1 / 2} \subset \mathfrak{g}_{-1 / 2}$ and $j \mathfrak{g}_{-1 / 2} \subset \mathfrak{g}_{-1 / 2}+\mathfrak{g}^{\prime}$. From this it follows $\mathfrak{u}_{1 / 2} \subset \mathfrak{g}_{1 / 2}^{\prime}$, whence $\mathfrak{u}_{1 / 2}=\left[e, j \mathfrak{u}_{1 / 2}\right] \subset \mathfrak{H}_{1 / 2}$.

Since $\mathfrak{H}_{1 / 2} \subset \mathfrak{n}_{1 / 2}$ we know that every $h_{1 / 2} \in \mathfrak{h}_{1 / 2}=\mathfrak{g}_{1 / 2} \cap \mathfrak{h}$ has a non-zero component in $\mathfrak{w}_{1 / 2}$. This implies that $\mathfrak{h} \cap \mathfrak{g}_{1 / 2}^{[1 / 2]}\left(c_{i}\right) \not \equiv 0 \bmod \mathfrak{n}$ for $c_{1}$ or for $c_{2}$. If $\mathfrak{h}_{1 / 2} \cap \mathfrak{g}^{[1 / 2]}\left(c_{i}\right) \not \equiv 0 \bmod \mathfrak{n}$ only for one of the idempotents $c_{1}, c_{2}$, then denote by $c$ the other idempotent. If this space is nontrivial for $c_{1}$ and for $c_{2}$, then choose $c=c_{1}$.

We consider the Kähler subalgebra $\tilde{\mathrm{g}}=\mathrm{g}^{[0]}(c)+\mathrm{g}^{[1]}(c)$; as mentioned above we know that for this algebra the Radical Conjecture holds. Since $e-c \in \tilde{\mathfrak{g}}$ we can form the weight space decomposition of $\tilde{\mathrm{g}}$ relative to $j(e-c)$. From our construction it follows that there exists a semisimple subalgebra $\tilde{\mathfrak{h}}$ of $\tilde{\mathfrak{g}}$ such that $\mathfrak{i} \subset \tilde{\mathfrak{g}}^{[0]}(e-c)$ and $\tilde{\mathfrak{h}} \cap \tilde{\mathfrak{g}}^{[1 / 2]}(e-c) \not \equiv 0 \bmod \operatorname{rad} \tilde{\mathfrak{g}}$ holds. But by the Radical Conjecture this is not possible. Hence we have shown

$$
d_{3}+d_{1}=0 \quad \text { or } d_{2}+d_{0}=0 .
$$

3.8. We refine the description of $f_{0}=j d+t_{0}+w_{0}$. We know that $t_{0}$ is a linear combination of elements of type $j q_{i}$, where $q_{i}$ is an idempotent of $t_{0}, t_{0}=\sum a_{i} j q_{i}$. Since $\left[f_{0}, q_{i}\right] a_{i} q_{i}$, we have $a_{i} \in\{0, \pm 1,-2\}$ by 3.4 . If $a_{i}=-2$, then there exists $x \in g_{1}$ such that $q_{i}=\left(\operatorname{ad} f_{-1 / 2}\right)^{2} x$. Then $q_{i} \in \mathfrak{n}$. 
Since $e \in \mathfrak{g}_{1}$ is the maximal idempotent in $\mathfrak{n}$, this case cannot occur. Hence

LEMmA. $t_{0}=j q_{1}-j q_{2}$ where $q_{i}$ are idempotents in $t_{0}$.

3.9. In this section we want to prove $d_{3}=0$. Otherwise $\left(\operatorname{ad} f_{-1 / 2}\right)^{3} d_{3}$ $\in \mathfrak{n}_{-1 / 2}$ is an eigenvector of $f_{0}$ for the eigenvalue -3 . We note that as in 2.9 we get $\mathfrak{g}_{0}=\mathfrak{g}+j \mathfrak{g}_{1}$ where $\mathfrak{g}=\left\{x \in \mathfrak{g}_{0} ;[x, e]=0\right\}$ is $j$-invariant. Since $q_{1}, q_{2}, w_{0} \in \mathfrak{g}$ it is easy to see that the Kähler algebra generated by $q_{1}, q_{2}$, $w_{0}$ acts symplectic on the abelian Kähler algebra $\mathfrak{v}=\mathfrak{n}_{-1 / 2}+j \mathfrak{n}_{-1 / 2}$. Note $j \mathfrak{n}_{-1 / 2}=\left[e, \mathfrak{n}_{-1 / 2}\right]$. Therefore, $j q_{1}$ and $j q_{2}$ have only the eigenvalues $0, \pm 1 / 2$ on $\mathfrak{b}$ and $w_{0}$ has no real eigenvalues on $\mathfrak{v}$. Next we consider the elements $j d_{3}$ and $j\left(d_{3}+d_{1}\right)$. We know that they leave the flat part of $\mathrm{g}^{\prime}$ invariant and have only the eigenvalues $0, \pm 1 / 2$ there. Hence, $f_{0}=2 j d_{3}+j\left(d_{3}+d_{1}\right)$ $+j q_{1}-j q_{2}+w_{0}$ cannot have the eigenvalue -3 on $\mathfrak{n}_{-1 / 2}$.

3.10. In this section we show $d_{2}=0$. Suppose not, then $\left(\operatorname{ad} f_{-1 / 2}\right)^{2} d_{2} \in \mathfrak{n}_{0}$ is an eigenvector of $f_{0} \mathrm{for}$ the eigenvalue -2 . Let $x \in \mathfrak{n}_{0}$ and write $x=$ $a_{0}+t_{0}+j x_{1}$ where $a_{0}$ is in the flat part $\mathfrak{w}_{0}$ of $\tilde{g}=\mathfrak{g}_{0}+\mathfrak{g}_{1}$ and $t_{0}$ is in the domain part $t_{0}$ of $\tilde{\mathfrak{g}}$. Note $\mathfrak{w}_{0}+\mathrm{t}_{0} \subset \mathfrak{g}$ (see 3.9). Then $\left[f_{0}, x\right]=-2 x$ implies $D(x)=0$ and $\left(j q_{1}-j q_{2}, t_{0}\right)=-2 t_{0},\left(j\left(2 d_{2}\right), j x_{1}\right)=-2 x_{1}$ follows, where $(\cdot, \cdot)$ denotes the product in the underlying unmodified algebra. But $j\left(2 d_{2}\right)$ has only the eigenvalues $\pm 1,0$ in $j g_{1}$, whence $x_{1}=0$. A similar argument shows that $j q_{1}-j q_{2}$ does not have the eigenvalue -2 in $t_{0}$. Hence $x$ is contained in the flat part $\mathfrak{w}_{0} \subset \mathfrak{g}_{0}$ of $\tilde{\mathfrak{g}}$. But there the idempotents $j d_{2}$, $j\left(d_{2}+q_{1}\right)$ and $j q_{2}$ have only the eigenvalues $0, \pm 1 / 2$. Hence -2 cannot be obtained.

3.11. By the last sections we have only to consider the cases $d_{1}=e$ and $d_{0}=e$. In this section we consider the case $d_{0}=e$ and $f_{0}=w_{0} \in \mathfrak{w}_{0}$. This is impossible as follows from

LEMma. Let $w_{0} \in \mathfrak{w}_{0}$ such that ad $\mathfrak{w}_{0}$ is semisimple and has only real eigenvalues. Then ad $w_{0}=0$.

Proof. From [7; Chap. III, Lemma 9] we know

$$
\frac{d}{d t} \rho\left(e^{t \operatorname{ad} w_{0}} u, e^{t \operatorname{ad} w_{0}} v\right)=\rho\left(w_{0}, e^{t \operatorname{ad} w_{0}}[u, v]\right) .
$$

Since $w_{0} \in \mathfrak{g}_{0}$, only the component of $[u, v]$ in $\mathfrak{g}_{0}+\mathfrak{g}_{1}$ wwill contribute to the right hand side by [6; Corollary 4.22]. Using the induction hypothesis 
shows that only the component in $g_{0}$ can contribute. But we know $\left[w_{0}, a_{0}+j x_{1}\right]=\left(w_{0}, j x_{1}\right)-D\left(a_{0}+j x_{1}\right) w_{0}$, whence $\left(\operatorname{ad} w_{0}\right)^{2} \mid g_{0}=0$. Since ad $w_{0}$ is semisimple we obtain

$$
\operatorname{ad} w_{0} \mid g_{0}=0 .
$$

Therefore the right hand side of (1) is just $\rho\left(w_{0},[u, v]\right)$. An integration yields

$$
\rho\left(e^{t \operatorname{ad} w_{0}} u, e^{t \operatorname{ad} w_{0}} v\right)=t \rho\left(w_{0},[u, v]\right)+\rho(u, v) .
$$

By assumption ad $w_{0}$ is semisimple with only real eigenvalues. Let $u=u_{\lambda}$, $v=v_{\mu}$ be eigenvectors for ad $w_{0}$. Then (3) yields $e^{t(\lambda+\mu)} \rho(u, v) \doteq t \rho\left(w_{0},[u, v]\right)$ $+\rho(u, v)$. This implies

$$
\rho\left(w_{0},[u, v]\right)=0 \quad \text { for all } u, v \in \mathfrak{g} .
$$

This shows that ad $w_{0}$ is symplectic on $\mathrm{g}$. Moreover, $\left[j w_{0}, w_{0}\right]=0$ by (2). Now we apply the proof of [7; chap. II, Lemma 3]. Let $A(x)$ denote the $j$-linear part of ad $x, x \in R w_{0}+R j w_{0}$, and $B(x)$ the $j$-antilinear part. As in loc. cit. one shows

$$
B\left(j w_{0}\right)=j B\left(w_{0}\right) \text { and } 2 B\left(w_{0}\right)^{2}=\left[j A\left(j w_{0}\right), A\left(w_{0}\right)\right] .
$$

This yields trace $B\left(w_{0}\right)^{2}=0$. Finally, since ad $w_{0}$ is symplectic, it is easy to see that $B\left(w_{0}\right)$ is selfadjoint and $A\left(w_{0}\right)$ is skewadjoint relative to $\langle u, v\rangle=\rho(j u, v)$ modulo $\mathfrak{f}$. Altogether this implies $B\left(w_{0}\right) \mathfrak{g} \subset \mathfrak{l}$. Thus ad $w_{0}=A\left(w_{0}\right)$ is skewadjoint on $\mathfrak{g} / \mathfrak{f}$, whence ad $w_{0} \mathfrak{g} \subset \mathfrak{f}$, since the eigenvalues of ad $w_{\mathrm{e}}$ are assumed to be real. From this the lemma follows

3.12. In this section we exclude the case $e=d_{1}$ and $f_{0}=j e+w_{0}$.

Lemma. The case $f_{0}=j e+w_{0}$ does not occur.

Proof. We note first that ad $w_{0} \mid g_{0}$ is skewadjoint since $\left[w_{0}, j e\right]=0$ and adje $\mid g_{0}$ is semisimple as well as $\operatorname{ad} f_{0}=\operatorname{ad} w_{0}+\operatorname{adje.~}$

Let $\mathfrak{u}^{[\lambda]}$ denote eigenspace for the eigenvalue $\lambda$ of the real part of $\operatorname{ad} w_{0}$ on $\mathfrak{u}=\mathfrak{g}_{1 / 2}+\mathfrak{g}_{-1 / 2}$.

We start again from the equation

$$
\frac{d}{d t} \rho\left(e^{t \operatorname{ad} w_{0}} u, e^{t \operatorname{ad} w_{0}} v\right)=\rho\left(w_{0}, e^{t \operatorname{ad} w_{0}}[u, v]\right) .
$$

As before we only have to consider the component of $[u, v]$ in $g$. But 
from above we have ad $w_{0}\left|g_{0}=D\left(w_{0}\right)\right| g_{0}$ where $D\left(w_{0}\right)$ is the modification derivation of $w_{0}$ in $g_{0}+g_{1}$. This implies that the right hand side of (1) is $\rho\left(w_{0},[u, v]\right)$. Therefore an integration yields

$$
\rho\left(e^{t \operatorname{ad} w_{0}} u, e^{t \operatorname{ad} w_{0}} v\right)=t a+b .
$$

For $u \in \mathfrak{u}^{[\lambda]}, v \in \mathfrak{u}^{[\mu]}, \lambda+\mu \neq 0$ the left side grows here like $e^{t(\lambda+\mu)}$ and the right side is polynomial. This is a contradiction. Hence we obtain

$$
\rho\left(\mathfrak{u}^{[\lambda]}, \mathfrak{H}^{[\mu]}\right)=0 \quad \text { if } \lambda+u \neq 0 .
$$

From above we know that ad $f_{0}$ attains only the eigenvalue 0 in $g_{0}$. Hence, from 3.4 we derive that ad $f_{0}$ can only have the eigenvalues -1 , 2,0 in $\mathfrak{g}_{1 / 2}$ and $-2,0$ in $\mathfrak{g}_{-1 / 2}$. Since adje has the weight $1 / 2$ and $-1 / 2$ there respectively, the real part of ad $w_{0}$ has the eigenvalues $-3 / 2,3 / 2$, $-1 / 2$ in $g_{1 / 2}$ and $-3 / 2,1 / 2$ in $g_{-1 / 2}$ (in the same order as above). Since $\rho$ is nondegenerate on $\mathfrak{u}=\mathfrak{g}_{1 / 2}+\mathfrak{g}_{-1 / 2}$ we derive from (3) that the weight spaces with opposite signs have the same dimension. Therefore $\operatorname{dim} \mathfrak{g}_{1 / 2}^{[3 / 2]}$ $=\operatorname{dim} \mathfrak{g}_{1 / 2}^{[-3 / 2]}+\operatorname{dim} \mathfrak{g}_{-1 / 2}^{[-3 / 2]}$ and $\operatorname{dim} \mathfrak{g}_{1 / 2}^{(2)}=\operatorname{dim} \mathfrak{g}_{1 / 2}^{[3 / 2]}=\operatorname{dim} \mathfrak{g}_{-1 / 2}^{(-2)}=\operatorname{dim} \mathfrak{g}_{-1 / 2}^{[-3 / 2]}$ where we have used 3.4 and the notation $\mathfrak{g}_{*}^{(\lambda)}$ for the eigenspaces of ad $f_{0}$ in $\mathfrak{g}_{*}$. But then, again by 3.4 , we have $0=\operatorname{dim} \mathfrak{g}_{1 / 2}^{[-3 / 2]}=\operatorname{dim} \mathfrak{g}_{1 / 2}^{(-1)}=\operatorname{dim} \mathfrak{g}_{1}^{(1)}=\operatorname{dim} \mathfrak{g}_{1}$. This is a contradiction, proving the lemma.

3.13. In this section we start to look at $g_{0}$ more closely.

Using the induction hypothesis we see that $\mathfrak{w}_{0}+\mathfrak{i}$ and $j \mathfrak{g}_{1}+\mathfrak{t}_{0}+\mathfrak{f}$ are subalgebras of $\mathfrak{g}_{0}, \mathfrak{w}_{0}+\mathfrak{l}$ is $j$-invariant. By 1.4 , we can even assume (after an inessential change of $j$ ) that $\left[\mathfrak{f}, \mathfrak{w}_{0}\right] \subset \mathfrak{w}_{0}$ holds. We can write $\mathfrak{f}=\mathfrak{f}_{a}+\mathfrak{f}_{0}+\mathfrak{f}_{1}$ such that $\mathfrak{l}_{1}+j \mathfrak{g}_{1}$ and $\mathfrak{f}_{0}+\mathfrak{t}_{0}$ are subalgebras where $\mathfrak{f}_{i}$ does not contain any ideal of the corresponding algebra. Moreover, we can assume that $\left[\mathfrak{f}_{0}, \mathfrak{f}_{0}+\mathfrak{t}_{0}+\mathfrak{f}_{1}+j \mathfrak{g}_{1}\right]=0$ holds. This implies that $\left(\mathfrak{f}_{1}+j \mathfrak{g}\right)+$ $\left(\mathfrak{f}_{0}+\mathfrak{t}_{0}\right)$ contains a maximal noncompact semisimple subalgebra $\mathfrak{h}_{0 s}$ of $\mathfrak{g}_{0}$. We can assume that a Cartan algebra of $\mathfrak{h}_{0 s}$ is contained in the span of the $j c, c$ a minimal idempotent of $t_{0}+\mathfrak{g}_{1}+j \mathfrak{g}_{1}$. From this it is easy to derive that $h_{0 s}$ is contained in the subspace $\mathfrak{h}_{0}$ of the maximal semisimple subalgebra $\mathfrak{h}=\mathfrak{h}_{-1 / 2}+\mathfrak{h}_{0}+\mathfrak{h}_{1 / 2}$ of $\mathfrak{g}$ considered in 3.3. Clearly we have $\mathfrak{h}_{0 s}=\mathfrak{h}_{0 s}^{0} \oplus \mathfrak{h}_{0 s}^{1}$ where $\mathfrak{h}_{0 s}^{0} \subset \mathfrak{l}_{0}+\mathfrak{t}_{0}$ and $\mathfrak{h}_{0 s}^{1} \subset \mathfrak{f}_{1}+j \mathfrak{g}_{1}$.

Assume $\mathfrak{b}=\left[\mathfrak{h}_{-1 / 2}, \mathfrak{h}_{1 / 2}\right] \cap\left(\mathfrak{f}_{1}+j \mathfrak{g}_{1}\right)$ contains a nontrivial, noncompact simple subalgebra $\mathfrak{h}^{\prime \prime}$. We can assume that $\mathfrak{h}^{\prime \prime}$ is maximal in $\mathfrak{b}$ and an ideal of $\mathfrak{b}$. Then there exists a simple ideal $\mathfrak{h}^{\prime} \subset \mathfrak{h}, \mathfrak{h}^{\prime}=\mathfrak{h}_{-1 / 2}^{\prime}+\mathfrak{h}_{0}^{\prime}+\mathfrak{h}_{1 / 2}^{\prime}$ satisfying $\mathfrak{h}^{\prime \prime} \subset \mathfrak{h}_{0}^{\prime}$ and $\mathfrak{h}_{ \pm 1 / 2}^{\prime} \neq 0$. Since $\mathfrak{h}^{\prime \prime} \neq 0$ we can choose $f_{0} \in \mathfrak{h}_{0}^{\prime}$ so 
that it has a nontrivial component $f_{0}^{\prime \prime}$ in $\mathfrak{h}^{\prime \prime}$. But we have reduced the discussion before to the case $f_{0}=\lambda j e+j q^{\prime}+w_{0}$ where $\lambda=0,1, q^{\prime} \in \mathfrak{t}_{0}$, $w_{0} \in \mathfrak{w}_{0}$. This shows that $f_{0}$ commutes with $\mathfrak{h}^{\prime \prime}$ on $\mathfrak{g}_{1}$, whence $f_{0}^{\prime \prime}=0$. This is a contradiction and implies that $\mathfrak{h}_{0 s}^{1}$ commutes with $\left[\mathfrak{h}_{-1 / 2}, \mathfrak{h}_{1 / 2}\right]$ and with $\mathfrak{h}_{-1 / 2}+\mathfrak{h}_{1 / 2}$. Moreover, $\left[\mathfrak{h}_{-1 / 2}, \mathfrak{h}_{1 / 2}\right] \subset \mathfrak{l}_{0}+\mathfrak{t}_{0}+\mathfrak{l}$ holds.

3.14. We continue investigating $g_{0}$ by considering $\operatorname{rad}\left(\mathfrak{g}_{0}\right)$.

First we prove

LEMMA. $\operatorname{rad}\left(\left[\mathfrak{g}_{0}, g_{0}\right]\right) \subset \operatorname{nil}(\mathfrak{g})$.

Proof. The maximal semisimple subalgebra $\mathfrak{h}$ under consideration can be written as sum of ideals $\mathfrak{h}=\mathfrak{h}^{*} \oplus \tilde{\mathfrak{h}}$ where $\mathfrak{h}^{*}=\mathfrak{h}_{-1 / 2}+\left[\mathfrak{h}_{-1 / 2}, \mathfrak{h}_{1 / 2}\right]+\mathfrak{h}_{1 / 2}$. By construction, $\left[\mathfrak{h}_{-1 / 2}, \mathfrak{h}_{1 / 2}\right]+\tilde{\mathfrak{h}} \subset \mathfrak{g}_{0}$ and $\left[\mathfrak{h}_{-1 / 2}, \mathfrak{h}_{1 / 2}\right]=\mathfrak{h}_{0}^{\prime}+\mathfrak{z}$ is a reductive Lie algebra with center $z$ and semisimple part $\mathfrak{h}_{0}^{\prime}$. It is clear that $\mathfrak{g}_{0}=$ $\mathfrak{h} \cap \mathfrak{g}_{0}+(\operatorname{rad}(\mathfrak{g}))_{0}$ holds, where $(\operatorname{rad}(\mathfrak{g}))_{0}=\mathfrak{g}_{0} \cap \operatorname{rad}(\mathfrak{g})$. Therefore $\operatorname{rad}\left(\mathfrak{g}_{0}\right)=$ $\mathfrak{z}+(\operatorname{rad}(\mathfrak{g}))_{0}$ and $\left[\mathfrak{g}_{0}, \mathfrak{g}_{0}\right]=\mathfrak{h}_{0}^{\prime}+\tilde{\mathfrak{h}}+\left[\mathfrak{g}_{0},(\operatorname{rad}(\mathfrak{g}))_{0}\right]$. Since $\mathfrak{h}_{0}^{\prime}+\tilde{\mathfrak{h}}$ is semisimple and $\mathfrak{v}=\left[\mathfrak{g}_{0},(\operatorname{rad} \mathfrak{g})_{0}\right]$ is a solvable ideal, we have $\mathfrak{v}=\operatorname{rad}\left(\left[\mathfrak{g}_{0}, \mathfrak{g}_{0}\right]\right)$. Clearly, $\mathfrak{v} \subset \operatorname{nil}(\mathrm{g})$. Hence the claim.

3.15. We had chosen $\mathfrak{n}$ to be maximal in $\mathfrak{r}$. Therefore, since [nil(g), $\mathfrak{r}$ ] $\varsubsetneqq \mathfrak{x}$ is an ideal of $\mathfrak{g}$, we can - and will - assume that $\mathfrak{n} \supset[\operatorname{nil}(\mathfrak{g}), \mathfrak{x}]$ holds.

Moreover, if $x$ is an element or a subspace of $x$ which is invariant under the family $\mathfrak{b}$ of endomorphisms chosen in 3.3 , and if $\mathfrak{u}(x)$ is the $\mathfrak{h}$-module generated by $x$, then $\mathfrak{u}(x)+\mathfrak{n} \subset \mathfrak{x}$ is an ideal of $\mathfrak{g}$ and $\mathfrak{x}=\mathfrak{u}(x)$ $+\mathfrak{n}$ follows.

3.16. To complete the proof of this "Case 3" we need detailed information on $t_{0}+\mathfrak{f}$. We recall that, by the induction hypothesis, $t_{0}+\mathfrak{f}$ is a $j$-invariant subalgebra of $g_{0}$ which corresponds to a homogeneous Siegel domain.

LEMma. $\operatorname{nil}\left(t_{0}+\mathfrak{f}\right) \subset \operatorname{rad}\left(\left[\mathfrak{g}_{0}, g_{0}\right]\right) \subset \operatorname{nil}(\mathfrak{g})$.

Proof. It is clear by 3.14 that we only have to prove the first inclusion. Since nil $\left(t_{0}+\mathfrak{f}\right)$ is invariant under modification derivations and since the nilradical does not change when considering the algebraic hull of $\mathfrak{g}_{0}+\mathfrak{g}_{1}$, we can assume that $\mathfrak{g}_{0}+\mathfrak{g}_{1}$ is algebraic and $\mathfrak{w}_{0}+\mathfrak{t}_{0}$ is split solvable. But then $\left[\mathfrak{f}, \mathfrak{w}_{0}\right] \subset \mathfrak{w}_{0}$ and nil $\left(\mathfrak{t}_{0}+\mathfrak{l}\right)$ is a solvable ideal of $\mathfrak{g}_{0}$. Therefore $\operatorname{nil}\left(t_{0}+\mathfrak{f}\right) \subset \operatorname{rad}\left(\left[g_{0}, g_{0}\right]\right)$ as claimed. 
3.17. An application of the last section yields

Lemma. $\mathfrak{t}_{0}$ corresponds to a symmetric tube domain.

Proof. Let $q$ be the maximal idempotent of $\mathfrak{t}_{0}$. Then $\mathfrak{u}=\mathfrak{g}_{0}+\mathfrak{g}_{1}$ splits into $\mathfrak{u}_{1}+\mathfrak{u}_{1 / 2}+\mathfrak{u}_{-1 / 2}+\mathfrak{u}_{0}$ relative to $j q$ where $\mathfrak{g}_{1}+j \mathfrak{g}_{1} \subset \mathfrak{u}_{0}$. Set $\mathfrak{r}_{u}=\mathfrak{r} \cap \mathfrak{u}$. We have seen in [6; Lemma 4.26] that $\mathfrak{u}=\mathfrak{r}_{u}+j \mathfrak{x}_{u}+\mathfrak{l}$ holds. Clearly $\mathfrak{r}_{u}=\oplus\left(\mathfrak{r}_{u}\right)_{\lambda}$ where $\left(\mathfrak{r}_{u}\right)_{\lambda}=\mathfrak{x}_{u} \cap \mathfrak{H}_{\lambda}$. We can use the proof of $[6 ; 4.10]$ and obtain $\mathfrak{u}_{1}=\left(\mathfrak{x}_{u}\right)_{1}$. Moreover, since $j \mathfrak{u}_{-1 / 2}=\left[q, \mathfrak{u}_{-1 / 2}\right], j \mathfrak{u}_{-1 / 2} \subset\left(\mathfrak{x}_{u}\right)_{1 / 2}$ holds. Therefore, if $t_{0} \cap \mathfrak{u}_{1 / 2} \neq 0$, then $t_{0} \cap\left(\mathfrak{r}_{u}\right)_{1 / 2} \neq 0$. But since $t_{0}+\mathfrak{t}_{1 / 2} \subset \operatorname{nil}\left(t_{0}+\mathfrak{f}\right)$ this implies $\left[j\left(\mathrm{t}_{0} \cap\left(\mathfrak{r}_{u}\right)_{1 / 2}\right), \mathrm{t}_{0} \cap\left(\mathfrak{r}_{u}\right)_{1 / 2}\right] \subset\left[\operatorname{nil}\left(\mathrm{t}_{0}+k\right), \mathfrak{x} \cap \mathfrak{g}_{0}\right] \subset[\operatorname{nil}(\mathfrak{g}), \mathfrak{x}] \subset \mathfrak{n}$ by 3.15 and 3.16. Therefore $\mathfrak{n} \cap \mathfrak{u}_{1} \neq 0$. But then $\mathfrak{u}_{1}$ contains an idempotent which is already contained in $\mathfrak{n}$. This is a contradiction to the choice of $e$. Hence $t_{0} \cap \mathfrak{u}_{1 / 2}=0$. This proves that $t_{0}$ corresponds to a tube domain.

To finish the proof it suffices to show that $\left(t_{0}+\mathfrak{l}\right) \cap \mathfrak{u}_{0}$ is reductive. But otherwise $\mathfrak{v}=\operatorname{nil}\left(\mathfrak{t}_{0}+\mathfrak{l}\right) \neq 0$. Since $\mathfrak{b}$ is invariant under all $j v_{i}, v_{i}$ a minimal idempotent of $\mathfrak{t}_{0}$, we obtain $\mathfrak{b}=\oplus \mathfrak{b}_{i j}$ where $\mathfrak{b}_{i j}=\left(t_{0}\right)_{i j}$. Note $\left[\mathfrak{b}_{i j}, \mathfrak{u}_{1}\right] \subset[\operatorname{nil}(\mathrm{g}), \mathfrak{x}] \subset \mathfrak{n}$ by 3.15 and 3.16. Finally, if $\mathfrak{v}_{i j} \neq 0$, then it is easy to see that $\left[\mathfrak{b}_{i j}, \mathfrak{u}_{1}\right] \subset \mathfrak{n}$ contains an idempotent of $\mathfrak{n}$, again a contradiction. This proves the lemma.

3.18. Let $\mathfrak{h}=\mathfrak{h}^{*} \oplus \tilde{\mathfrak{h}}$ where $\mathfrak{h}^{*}=\mathfrak{h}_{-1 / 2}+\left[\mathfrak{h}_{-1 / 2}, \mathfrak{h}_{1 / 2}\right]+\mathfrak{h}_{1 / 2}$ and $\tilde{\mathfrak{h}} \subset \mathfrak{g}_{0}$ is an ideal of $\mathfrak{h}$. We set $\mathfrak{h}_{\lambda}^{*}=\mathfrak{h}^{*} \cap \mathfrak{g}_{\lambda}$.

LEMMA. Let $\tilde{\mathfrak{h}}_{i}$ be a simple noncompact summand of $\tilde{\mathfrak{h}}$ satisfying $\tilde{\mathfrak{h}}_{i} \subset \mathfrak{t}_{0}+$ I. Then

a) $\tilde{\mathfrak{h}}_{i}$ is the noncompact part of $\tilde{\mathfrak{h}} \cap\left(\mathfrak{t}_{0}+\mathfrak{l}\right)$.

b) $\mathfrak{h}^{*} \cap \mathfrak{g}_{0}$ is reductive with compact semisimple part.

c) $t_{0}$ corresponds to the irreducible symmetric tube domain associated with $\tilde{\mathfrak{h}}_{i}$.

Proof. Denote by $\mathfrak{p}$ the ideal of $\mathfrak{t}_{0}+\mathfrak{f}$ associated with $\tilde{\mathfrak{h}}_{i}$. Then $\mathfrak{p}=\mathfrak{p}_{1}+\mathfrak{p}_{0}$ where $\mathfrak{p}_{1} \subset \mathfrak{x}, \mathfrak{p}_{0} \supset j \mathfrak{p}_{1}$ and $\mathfrak{p}_{1}$ is an $\tilde{\mathfrak{h}}_{i}$-module and invariant under b. Moreover, $\tilde{\mathfrak{h}}_{i}$ acts irreducibly on $\mathfrak{p}_{1}$ and trivially on all other ideals of $\mathfrak{t}_{0}+\mathfrak{f}$. Finally, $\tilde{\mathfrak{h}}$ commutes with $\mathfrak{h}_{\lambda}^{*}$ for $\lambda=0, \pm 1 / 2$ and $\left[\mathfrak{G}_{0}^{*}, \mathfrak{p}_{1}\right] \subset \mathfrak{p}_{1}$.

Set $X=\sum\left[\mathfrak{h}_{\varepsilon_{1}}\left[\mathfrak{h}_{\varepsilon_{2}}, \cdots,\left[\mathfrak{h}_{\varepsilon_{r}}, \mathfrak{p}_{1}\right] \cdots\right]\right.$ where $\varepsilon_{i} \in\{ \pm 1 / 2\}$ and $r \geq 0$. Then $X$ is an $\mathfrak{h}$-module and $X \subset \mathfrak{r}$. Since $\mathfrak{p}_{1} \subset X$ we have $X \not \subset \mathfrak{n}$ and $\mathfrak{x}=\mathfrak{n}+X$ follows. Clearly, $X=X_{-1 / 2}+X_{0}+X_{1 / 2}+X_{1}$ where $X_{\lambda}=X \cap g_{\lambda}$ and $X_{0}$ corresponds to the summands satisfying $\varepsilon_{1}+\cdots+\varepsilon_{r}=0$. Since $\tilde{\mathfrak{h}}_{i}$ commutes with $\mathfrak{h}_{ \pm 1 / 2}=\mathfrak{h}_{ \pm 1 / 2}^{*}, X_{0}$ is a sum of irreducible $\tilde{\mathfrak{h}}_{i}$-modules isomorphic 
to $\mathfrak{p}_{1}$.

Next we note that $\mathfrak{n} \cap \mathfrak{g}_{0} \subset \mathfrak{w}_{0}+j \mathfrak{g}_{1}$ holds. Otherwise there exists some $n \in \mathfrak{n} \cap \mathfrak{g}_{0}, n=a+t+j x$, where $a \in \mathfrak{w}_{0}, x \in \mathfrak{g}_{1}$ and $0 \neq t \in \mathfrak{t}_{0}$. It is easy to see that we can even assume $t \in\left(t_{0}\right)_{1}$ where $\left(t_{0}\right)_{k}, k=0,1 / 2,1$, are the weight spaces in $t_{0}$ of a maximal idempotent of $t_{0}$. Since $\mathfrak{n}$ is invariant under all $\operatorname{Re}(\operatorname{ad} j c), c$ a minimal idempotent in $t_{0}$, we can even assume $\mathfrak{n} \cap\left(t_{0}\right)_{1}=0$. But now it is easy to derive that $\mathfrak{n}$ contains a minimal idempotent of $t_{0}$. This is a contradiction since $e$ was chosen maximal in $\mathrm{g}^{\prime}$.

Now suppose $R=\operatorname{Re}(\operatorname{adj} c)$ where $c$ is the maximal idempotent of some summand $\mathfrak{v}$ of $t_{0}+\mathfrak{l}$ which is different from the one associated with $\tilde{\mathfrak{h}}_{i}$. Then $R \mathfrak{h}_{ \pm 1 / 2} \subset \mathfrak{h}_{ \pm 1 / 2}$ by our choice of $\mathfrak{h}$ (see 3.3) and $R \mathfrak{p}_{1}=0$. Hence $R X \subset X$. Since $R$ and ad $\tilde{\mathfrak{h}}_{i}$ commute, $R X$ is also an $\tilde{\mathfrak{h}}_{i}$-module. Moreover, the eigenspaces $X_{0}^{(r)}$ of $R$ in $X_{0}$ are $\tilde{\mathfrak{h}}_{i}$-modules. By the remark above they are even a sum of modules isomorphic to $\mathfrak{p}_{1}$. Therefore, since $X_{0}^{(1)} \subset \mathfrak{v}, X_{0}^{(1)}=0$. This shows that $\mathfrak{x}=X+\mathfrak{n}$ has no component in $R \mathfrak{v}$. But we have seen in the proof of 3.17 that $\mathfrak{x} \cap R \mathfrak{v} \neq 0$ holds. This is a contradiction, proving the lemma.

3.19. We consider $\mathfrak{p}=\mathfrak{t}_{0}+\mathfrak{t}$ more closely. First we split $\mathfrak{p}=\oplus_{i} \mathfrak{p}_{0}$ $+\mathfrak{f}_{0}$ where each $i \mathfrak{p}$ corresponds to an irreducible symmetric tube domain and $\mathfrak{f}_{0}$ is an ideal of $\mathfrak{p}$ contained in $\mathfrak{H}_{\text {. Hence }} \mathfrak{i}={ }_{i} \mathfrak{p}_{0}+{ }_{i} \mathfrak{p}_{1}$ and ${ }_{i} \mathfrak{p}_{1}$ contains a maximal idempotent $p_{i}$ of ${ }_{i} p$.

Let $\mathfrak{h}_{i}^{*}={ }_{i} \mathfrak{h}_{-1 / 2}+{ }_{i} \mathfrak{h}_{0}^{*}+{ }_{i} \mathfrak{h}_{1 / 2}$ be a simple summand of $\mathfrak{h}^{*}$. Then $\left(\mathfrak{h}_{1 / 2},{ }_{i} \mathfrak{h}_{-1 / 2}\right)$ carries naturally the structure of a simple Jordan pair [11; chapter II]. Using a Cartan involution of this Jordan pair $[12 ; \S 5]$, we even get the structure of a compact Jordan triple system on $V={ }_{i} \mathfrak{h}_{1 / 2}[12 ; \S 5]$. Then ${ }_{i} \xi_{0}^{*}$ is the "structure algebra" of $V$.

Lemma. The Jordan triple $V$ has rank $V \leq 1$.

Proof. Suppose $V$ has rank $\geq 2$, then there exist at least two minimal orthogonal idempotents $v_{1}, v_{2}$ of $V$. Using the Peirce decomposition of $V$ relative to $v_{1}, v_{2}$ it is easy to see that $g l(2, R) \subset{ }_{i} \zeta_{0}^{*}$ such that its offdiagonal parts are contained in some rootspace of ${ }_{i} \mathfrak{G}_{0}^{*}$ (relative to some maximal $\boldsymbol{R}$-split toral subalgebra). We can choose (different) subalgebras $s l(2, R)$ of $\mathfrak{h}$ so that the corresponding Cartan algebras are spanned by $f_{0}, f_{0}^{\prime}$ and $f_{0}+f_{0}^{\prime}$ which corresponds to the matrices $E_{11}=\operatorname{diag}\{1,0\}$, $E_{22}=\operatorname{diag}\{0,1\}$ and $E=\operatorname{diag}\{1,1\}$. These facts can be derived easily from 
$[15 ; \mathrm{IV}, \S 2]$. Since $f_{0}$ and $f_{0}^{\prime}$ have only the eigenvalues $0, \pm 1$, on ${ }_{i} \mathfrak{p}$ and since $g l(2, R)$ splits into rootspaces of ${ }_{i} \mathfrak{h}_{0}^{*}$ it is easy to see that there exist minimal idempotents $c_{1}, c_{2}$ in ${ }_{i} \mathfrak{p}_{1}$ such that $f_{0}=\lambda j e+j c_{1}-j c_{2}-j q$ $+r, f_{0}^{\prime}=\lambda j e+j c_{2}-j c_{1}-j q+r^{\prime}$, where $\lambda \in\{0,1\}, q=p_{i}-c_{1}-c_{2}$ and $r^{\prime}$ acts trivially on $i p_{1}$. Since $f_{0}+f_{0}^{\prime}$ has also only the eigenvalues $0, \pm 1$ on ${ }_{i} p_{1}, \lambda=0$ follows. Similarly we get $q=0$. This implies in particular, that the cone corresponding to $i p$ has rank $\leq 2$. But then $f_{0}+f_{0}^{\prime}$ centralizes, ${ }_{i} \mathfrak{h}_{0}^{*}$ whence $f_{0}+f_{0}^{\prime}$ has only the eigenvalues \pm 2 on ${ }_{i} \mathfrak{h}_{ \pm 1 / 2}$. Moreover, $\left.\operatorname{ad}\left(f_{0}+f_{0}^{\prime}\right)\right|_{i p_{1}}=0$ and there exists a $k$ such that $\left.\operatorname{ad}\left(f_{0}+f_{0}^{\prime}\right)\right|_{k} p_{1} \neq 0$. We recall that $\operatorname{ad}\left(f_{0}+f_{0}^{\prime}\right)$ has only the eigenvalues $0, \pm 1$ in $g_{0}$.

Consider the vector space $U$ spanned by "monomials" of type $\left[r_{1} \mathfrak{h}_{\varepsilon_{1}},\left[r_{2} \mathfrak{h}_{\varepsilon_{2}}, \cdots,{ }_{i} \mathfrak{p}_{1}\right] \cdots\right]$, where $r_{k}$ is arbitrary, $\varepsilon_{t}= \pm 1 / 2$ and $i$ is fixed. It is easy to see that $U$ is an $\mathfrak{h}$-module and invariant under $\mathfrak{b}$. Moreover, $f_{0}+f_{0}^{\prime}$ has only the eigenvalues $0, \pm 2$ on $U$. Hence $\operatorname{ad}\left(f_{0}+f_{0}^{\prime}\right) \mid U \cap g_{0}=0$. This implies that $U$ has no component in ${ }_{k} \mathfrak{p}_{1}$, if $\left.\operatorname{ad}\left(f_{0}+f_{0}^{\prime}\right)\right|_{k} \mathfrak{p}_{1} \neq 0$. As in the proof of 3.18 we consider $\mathfrak{r}=U+\mathfrak{n}$ and obtain a contradiction, since ${ }_{k} \mathfrak{p}_{1} \subset \mathfrak{x}$. This proves the lemma.

3.20. We continue the investigations of the last section. We assume that $\mathfrak{h}$ has a simple subalgebra ${ }_{i} \mathfrak{h}={ }_{i} \mathfrak{h}_{-1 / 2}+{ }_{i} \mathfrak{h}_{0}+{ }_{i} \mathfrak{h}_{1 / 2}$ such that ${ }_{i} \mathfrak{G}_{0}$ has a noncompact simple subalgebra. We have seen in the last section that $V={ }_{i} \mathfrak{h}_{1 / 2}$, considered as Jordan triple, has rank $V=1$.

$V$ is said to be of "algebra type" if there exists some subalgebra $s l(2, \boldsymbol{R})$ of ${ }_{i} \mathfrak{h}$ such that the corresponding $f_{0}$ has only the eigenvalue 2 on ${ }_{i} \mathfrak{h}_{1 / 2},-2$ or ${ }_{i} \mathfrak{G}_{-1 / 2}$ and 0 or ${ }_{i} \mathfrak{G}_{0}$.

LEMMA. $\quad V={ }_{i} \mathfrak{h}_{1 / 2}$ is of algebra type.

Proof. Suppose this is wrong, then our assumptions imply that there exists a subalgebra $\operatorname{sl}(3, \boldsymbol{R}) \cong \mathfrak{h}^{\prime} \subset_{i} \mathfrak{h}$ such that $\mathfrak{h}^{\prime}=\mathfrak{h}_{-1 / 2}^{\prime}+\mathfrak{h}_{0}^{\prime}+\mathfrak{h}_{1 / 2}^{\prime}$ where (we may assume w.r.g) $\mathfrak{h}_{-1 / 2}^{\prime} \cong\left\{\left(\begin{array}{ll}0 & 0 \\ a & 0\end{array}\right) ; a \in R^{2}\right\}, \quad \mathfrak{G}_{0}^{\prime} \cong\left\{\left(\begin{array}{cc}-\operatorname{tr}(A) & 0 \\ 0 & A\end{array}\right), A \in\right.$ $g l(2, R)\}, \quad \mathfrak{H}_{1 / 2}^{\prime} \cong\left\{\left(\begin{array}{ll}0 & b^{t} \\ 0 & 0\end{array}\right) ; b \in R^{2}\right\}$. Moreover, we can assume that the rootspaces of $\operatorname{sl}(3, \boldsymbol{R})$ are contained in rootspaces of ${ }_{i} \mathfrak{h}$ (relative to some maximal $R$-split toral subalgebra). This follows from [15] and [12; §3.2]. We consider the two copies of $\operatorname{sl}(2, \boldsymbol{R})$ inside $\mathfrak{h}^{\prime}=\operatorname{sl}(3, \boldsymbol{R})$ spanned by $f_{-1 / 2}, f_{0}, f_{1 / 2}$ and $\tilde{f}_{-1 / 2}, \tilde{f}_{0}, \tilde{f}_{1 / 2}$ respectively, where one has the following correspondences: $f_{1 / 2} \leftrightarrow(1,0), \tilde{f}_{1 / 2} \leftrightarrow(0,1), f_{-1 / 2} \leftrightarrow(1,0)^{t}, \tilde{f}_{-1 / 2} \leftrightarrow(0,1)^{t}, f_{0}=\left[f_{1 / 2}, f_{-1 / 2}\right]$ 
$\leftrightarrow \operatorname{diag}\{1,-1,0\}, \tilde{f}_{0}=\left[\tilde{f}_{1 / 2}, \tilde{f}_{-1 / 2}\right] \leftrightarrow \operatorname{diag}\{1,0,-1\}$. Moreciver, we know that the subalgebra $\operatorname{sl}(2, R) \subset \mathfrak{Y}_{0}^{\prime}$ acts on a selfdual cone in ${ }_{i} p$. We may assume that it acts in the natural way on a three dimensional subspace $H$ of ${ }_{i} p$ realized as $2 \times 2$ symmetric matrices. We denote $c_{1}=\operatorname{diag}\{1,0\}, c_{2}=$ $\operatorname{diag}\{0,1\}, x_{12}=\left(\begin{array}{ll}0 & 1 \\ 1 & 0\end{array}\right) \in H$. Since $f_{0}$ and $\tilde{f}_{0}$ are selfadjoint (with integral eigenvalues) we can label elements in $g$ by the pair of eigenvalues corresponding to $f_{0}$ and $\tilde{f}_{0}$ respectively. We also note that $f_{0}$ and $\tilde{f}_{0}$ have only the eigenvalues $0, \pm 1$, in $g_{0}$. Thus we may assume that $c_{1}$ belongs to $(1,-1), c_{2}$ to $(-1,1)$ and $x_{12}$ to $(0,0)$.

A straightforward computation in $\operatorname{sl}(3, R)$ shows $\left[f_{0}, f_{ \pm 1 / 2}\right]= \pm 2 f_{ \pm 1 / 2}$, $\left[f_{0}, \tilde{f}_{ \pm 1 / 2}\right]= \pm \tilde{f}_{1 / 2}$, and $\left[\tilde{f}_{0}, f_{ \pm 1 / 2}\right]= \pm f_{ \pm 1 / 2},\left[\tilde{f}_{0}, \tilde{f}_{ \pm 1 / 2}\right]= \pm 2 \tilde{f}_{ \pm 1 / 2}$. Moreover, for $x=\left[\tilde{f}_{-1 / 2}, f_{1 / 2}\right]$ we have $\left[x, \tilde{f}_{-1 / 2}\right]=0$ and $\left[x, c_{2}\right]=0$. The eigenvalues of $y=\left[f_{1 / 2}, c_{2}\right]$ are $(1,2)$. Since $c_{2}$ has eigenvalue -1 relative to $f_{0}, y \neq 0$. Also note that $y$ has eigenvalue 2 for $\tilde{f}_{0}$, whence $\left[\tilde{f}_{-1 / 2}, y\right] \neq 0$ and $\left[\tilde{f}_{-1 / 2},\left[\tilde{f}_{-1 / 2}, y\right]\right] \neq 0$. But $\left[\tilde{f}_{-1 / 2}, y\right]=\left[\tilde{f}_{-1 / 2},\left[f_{1 / 2}, c_{2}\right]\right]=\left[x, c_{2}\right]+\left[f_{1 / 2},\left[\tilde{f}_{-1 / 2}, c_{2}\right]\right]$ $=\left[f_{1 / 2},\left[\tilde{f}_{-1 / 2}, c_{2}\right]\right]$, hence $\left[\tilde{f}_{-1 / 2},\left[\tilde{f}_{-1 / 2}, y\right]\right]=\left[\tilde{f}_{-1 / 2},\left[f_{1 / 2},\left[\tilde{f}_{-1 / 2}, c_{2}\right]\right]\right]=\left[x,\left[\tilde{f}_{-1 / 2}, c_{2}\right]\right]$ $+\left[f_{1 / 2},\left[\tilde{f}_{-1 / 2},\left[\tilde{f}_{-1 / 2}, c_{2}\right]\right]\right]=0$, a contradiction. This proves the lemma.

3.21. By the results of the last sections we know that for each simple summand ${ }_{i} \mathfrak{h}={ }_{i} \mathfrak{G}_{-1 / 2}+{ }_{i} \mathfrak{h}_{0}+{ }_{i} \mathfrak{G}_{1 / 2}$ of $\mathfrak{h},{ }_{i} \mathfrak{G}_{ \pm 1 / 2} \neq 0$, on the space $V={ }_{i} \mathfrak{h}_{1 / 2}$ we obtain naturally the structure of a simple Jordan triple of algebra type and of rank 1. This implies [14; Lemma 2.1] that $V$ is isomorphic to a Jordan triple of a quadratic form $\left[\boldsymbol{R}^{n} ; \mathrm{Id}\right], n \geq 1$. Moreover, in all these cases the "structure algebra" ${ }_{i} \mathfrak{h}_{0}=\left[{ }_{i} \mathfrak{h}_{-1 / 2},{ }_{i} \mathfrak{h}_{1 / 2}\right]$ is isomorphic to $\boldsymbol{R} \oplus_{i} \mathfrak{f}$ where ${ }_{i} \mathfrak{f}=\mathfrak{i} \cap{ }_{i} \mathfrak{G}_{0}[13 ; \S 5]$. It is easy to see that ${ }_{i}^{\mathfrak{i}}=0$ if and only if ${ }_{i} \mathfrak{h} \cong \operatorname{sl}(2, R)$.

Finally, in all the cases except [R; Id] above, the Jordan triple $C$ is naturally a subtriple of $V$.

3.22. As a corollary of the last section we see that the noncompact part of $\left[\mathfrak{h}_{-1 / 2}, \mathfrak{h}_{1 / 2}\right]$ is also the center of this Lie algebra. In particular, a " $f_{0}$ " as considered before, contained in $\left[\mathfrak{h}_{-1 / 2}, \mathfrak{h}_{1 / 2}\right]$, commutes with $\mathfrak{h} \cap \mathfrak{g}_{6}$. Therefore such an $f_{0}$ has only the eigenvalues $0, \pm 2$ on $\mathfrak{h}$ (see also Lemma 3.20). Moreover, by 3.11 and 3.12 we can assume that $f_{0}$ has a nonvanishing " $q$-part".

Lemma. $f_{0}=\lambda j e+j q_{1}-j q_{2}+w_{0}$, where $\lambda \in\{0,1\}, q_{i}$ is a sum of maximal tripotents of irreducible factors of $t_{0}$ and $q_{1}+q_{2}$ is the maximal 
idempotent of $t_{0}$.

Proof. Suppose there exists an idempotent $0 \neq c \in \mathrm{t}_{0},\left[f_{0}, c\right]=0$, where $f_{0} \in \operatorname{sl}(2, \boldsymbol{R}) \cong \boldsymbol{R} f_{-1 / 2}+\boldsymbol{R} f_{0}+\boldsymbol{R} f_{1 / 2}$. Let $U$ be the $\mathfrak{h}$-module generated by $c$. It is easy to see that $f_{0}$ has only even integral eigenvalues on $U \cap g_{0}$. Hence $\left[f_{0}, U \cap \mathfrak{g}_{0}\right]=0$. As before we consider $\mathfrak{r}=\mathfrak{n}+U$ and see that no element of $\mathfrak{r}$ has a component in $\operatorname{Re}(\operatorname{ad} j q) t_{0}$. This is a contradiction since $\left[\operatorname{Re}(\operatorname{adj} q) \mathfrak{t}_{0}\right] \cap \mathfrak{x} \neq 0$ as shown in 3.17 and the assertion follows.

3.23. In this section we reduce further the possibilities for $\mathfrak{h}$.

Lemma. $\mathfrak{h}^{*}=\mathfrak{h}_{-1 / 2}+\left[\mathfrak{h}_{-1 / 2}, \mathfrak{h}_{1 / 2}\right]+\mathfrak{h}_{1 / 2}$ is simple.

Proof. Suppose there exist different simple summands ${ }_{1} \mathfrak{h}$ and ${ }_{2} \mathfrak{h}$ of $\mathfrak{h}^{*}$. By 3.20 we know that these Lie algebras have real rank one. Let $f_{1}, f_{2}$ be corresponding elements "of type $f_{0}$ ". Then, by Lemma 3.22, $f_{i}=\lambda_{i}$ je $+j q_{i}^{\prime}+w_{o i}$ for $i=1,2$. Since we can assume that also $f_{1}+f_{2}$ is "of type $f_{0}$ ", $\operatorname{ad}\left(f_{1}+f_{2}\right)$ has only the eigenvalues $0, \pm 1$ in $g_{0}$ and 0 or 1 in $\mathfrak{g}_{1}$. But $f_{1}+f_{2}=\left(\lambda_{1}+\lambda_{2}\right) j e+j\left(q_{1}^{\prime}+q_{2}^{\prime}\right)+\left(w_{01}+w_{02}\right)$ and $q_{1}^{\prime}+q_{2}^{\prime}=0$ follows. The remaining case $f_{1}+f_{2}=\lambda^{\prime} j e+w_{0}^{\prime}$ was already excluded in 3.11 and 3.12. This proves the lemma.

3.24. From 3.17 we know that $t_{0}$ corresponds to a tube domain. Hence $\mathfrak{t}_{0}=\mathfrak{u}+j \mathfrak{u}$ where $\mathfrak{u} \subset \mathfrak{x}$. By 3.18, if $\mathfrak{t}_{0}+\mathfrak{l}$ contains a noncompact semisimple ideal of $\mathfrak{h}$, then $\mathfrak{u}$ corresponds to an irreducible cone. Otherwise all occuring cones are one dimensional by 3.22 .

Lemma. $t_{0}$ corresponds to an irreducible symmetric tube domain.

Proof. Let $c$ be the maximal idempotent of an irreducible summand of $t_{0}$. By the remarks above we can assume that the corresponding cone is one dimensional. Since $\left[\mathfrak{h}_{0}, c\right] \subset R c$ we see that $U=\sum\left[\mathfrak{h}_{\varepsilon_{1}}\left[\cdots\left[\mathfrak{h}_{\varepsilon}, c\right] \cdots\right]\right.$, $\varepsilon_{i}= \pm 1 / 2$, is an $\mathfrak{h}$-module and invariant under $\mathfrak{b}$. We recall $\mathfrak{h}=\mathfrak{h}^{*}+\tilde{\mathfrak{h}}$ where $\mathfrak{h}^{*}=\mathfrak{h}_{-1 / 2}+\left[\mathfrak{h}_{-1 / 2}, \mathfrak{h}_{1 / 2}\right]+\mathfrak{h}_{1 / 2}$ and $\tilde{\mathfrak{h}} \subset \mathfrak{g}_{0}$. Moreover, by 3.23 , $\mathfrak{h}^{*}$ is simple. Since $R=\operatorname{Re}\left(\operatorname{ad} j c^{\prime}\right)$ leaves $\mathfrak{h}$ invariant by construction 3.3 , we see that for every idempotent $c^{\prime}$ of $\mathfrak{u}$ the derivation $R$ of $\mathfrak{h}$ is $s I$ for some value $s$ on $\mathfrak{h}_{1 / 2}$ and then $-s I$ on $\mathfrak{h}_{-1 / 2}$. Suppose that also $\left[j c^{\prime}, c\right]=0$ holds, then $\left[j c^{\prime}, U \cap \mathfrak{g}_{0}\right]=0$. Therefore $\mathfrak{r}=\mathfrak{n}+U$ has no component in $\boldsymbol{R} c^{\prime}$. But $c^{\prime} \in \mathfrak{u} \subset \mathfrak{r}$, a contradiction.

3.25. The above considerations restrict the possibilities for $\mathfrak{h}^{*}=\mathfrak{h}_{1 / 2}$ $+\left[\mathfrak{h}_{-1 / 2}, \mathfrak{h}_{1 / 2}\right]+\mathfrak{h}_{1 / 2}$ quite a bit. But we can even show 
LEMMA. $\mathfrak{h}^{*} \cong \operatorname{sl}(2, \boldsymbol{R})$.

Proof. Suppose this is wrong. Then from 3.21 it follows that $\operatorname{sl}(2, C)$ $\subset \mathfrak{h *}$. (Since $C$ is a subtriple of $V$ this follows from $[12 ; \S 3.2]$.) Moreover, the rootspaces of $\operatorname{sl}(2, C)$ are contained in rootspaces of $\mathfrak{h}^{*}$ (relative to some maximal $\boldsymbol{R}$-split toral subalgebra). We choose $f_{0}$ for the canonically embedded $\operatorname{sl}(2, R) \subset \operatorname{sl}(2, C)$. From 3.22 we know that $f_{0}$ is of type $f_{0}=\lambda j e+\varepsilon j q+w_{0}, \varepsilon= \pm 1$. Therefore, $f_{0}$ has the eigenvalue 0 on $\mathfrak{w}_{0}+$ $j \mathfrak{u}+\mathfrak{l}+j \mathfrak{g}_{1}$ and the eigenvalue $\varepsilon$ on $\mathfrak{u}$. We also note, that $f_{0}$ has on $\mathfrak{h}_{ \pm 1 / 2}$ the eigenvalue \pm 2 . Let $U$ denote the $\mathfrak{h}$-module generated by $\mathfrak{u}$. It is easy to see that $\left[\mathfrak{h}_{\alpha},\left[\mathfrak{h}_{\alpha}, \mathfrak{u}\right]\right]=0$ and $\left[\mathfrak{h}_{\alpha},\left[\mathfrak{h}_{-\alpha}, \mathfrak{u}\right]\right] \subset \mathfrak{u}$ for $\alpha= \pm 1 / 2$. This shows that $U=\mathfrak{u}+\left[\mathfrak{h}^{*}, \mathfrak{u}\right]$ holds. Let $\left[\mathfrak{h}_{-1 / 2}, \mathfrak{h}_{1 / 2}\right] \cong \boldsymbol{R} \oplus \mathfrak{f}^{*}$, where $\mathfrak{1}^{*} \subset \mathfrak{f}$. Then $\left[\mathfrak{f}^{*}, \mathfrak{u}\right]=0$ since $\mathfrak{u}$ is either one-dimensional or it is associated with some ideal $\tilde{\mathfrak{h}} \subset \mathfrak{g}_{0}$ of $\mathfrak{h}$. Let $W \subset U$ be an irreducible $\operatorname{sl}(2, C)$-submodule of $U$. Then $W=W_{0}+W_{\alpha}$ where $W_{\beta}=W \cap \mathfrak{g}_{\beta}$. Since $f_{0} \in \operatorname{sl}(2, C)$ and $W_{0} \subset \mathfrak{u}, W$ is not a trivial representation. Hence $W \cong C^{2}$. The subalgebra $\mathfrak{v}=$ $C \operatorname{diag}\{i,-i\}$ of $\operatorname{sl}(2, C)$ corresponds to a subalgebra of $\mathfrak{f}^{*}$. Considered as subalgebra of $\operatorname{sl}(2, C)$ it acts non-trivially on $W_{0}$, but as subalgebra of $\mathfrak{f}^{*}$ it acts trivially on $W_{0} \subset \mathfrak{u}$. This is a contradiction.

3.26. From 3.24 we know that the cone $C$ corresponding to $t_{0}$ is irreducible. Moreover, $C$ is not one-dimensional only if it is associated with an ideal $\tilde{\mathfrak{h}} \subset \mathfrak{g}_{0}$ of $\mathfrak{h}$. In this case we set $\tilde{\mathfrak{l}}=\tilde{\mathfrak{h}} \cap \mathfrak{f}$. Then $\tilde{\mathfrak{l}}$ is maximal compact in $\tilde{\mathfrak{h}}$.

LEmma. It suffices to consider the case where $C$ is one-dimensional.

Proof. Since $\mathfrak{h}^{*} \cong \operatorname{sl}(2, \boldsymbol{R}), \mathfrak{g}_{\alpha}=\boldsymbol{R} f_{\alpha}+\mathfrak{v}_{\alpha}$ where $\mathfrak{v}_{\alpha} \subset \operatorname{rad} \mathfrak{g}, a= \pm 1 / 2$. Therefore $\left[\mathfrak{g}_{-1 / 2}, \mathfrak{g}_{1 / 2}\right] \subset \boldsymbol{R} f_{0}+\operatorname{nil}(\mathfrak{g})$. We also note that $\tilde{\mathfrak{g}}_{0}=\mathfrak{w}_{0}+\mathfrak{t}_{0}+j \mathfrak{g}_{1}$ is a solvable subalgebra of $\mathfrak{g}_{0}, \tilde{\mathfrak{g}}_{0}+\mathfrak{l}=\mathfrak{g}_{0}$. It is easy to verify that $\tilde{\mathfrak{g}}=$ $\mathfrak{g}_{-1 / 2}+\tilde{\mathfrak{g}}_{0}+\mathfrak{g}_{1 / 2}+\mathfrak{g}_{1}$ is a Kähler subalgebra of $\mathfrak{g}$ satisfying $\tilde{\mathfrak{g}}+\mathfrak{f}=\mathfrak{g}$. Since $\tilde{g}_{0}$ is solvable, the maximal semisimple subalgebra of $\tilde{\mathfrak{g}}$ is $\mathfrak{h}^{*} \cong \operatorname{sl}(2, R)$. In particular there is no ideal of $\mathfrak{h}$ contained in $\tilde{\mathfrak{g}}_{0}$. Therefore, applying the previous sections to $\tilde{\mathfrak{g}}$ shows that we can assume that $t_{0}$ corresponds to a one dimensional cone.

3.27. From 3.25 we know $\mathfrak{h}^{*} \cong \operatorname{sl}(2, R)$. Let $f_{0}$ denote the canonical generator of the Cartan subalgebra of $\mathfrak{h}^{*}$. Then $f_{0}=\lambda j e+\varepsilon q+w_{0}$. From 3.26 it follows that we can assume $\mathfrak{U}=\boldsymbol{R} q$. Moreover, as in the proof of 3.25 we see that for the $\mathfrak{h}$-module $U$, generated by $\mathfrak{u}$ we have $U=$ 
$R q+R\left[f_{1 / 2}, q\right]$, if $\varepsilon=-1$ and $U=R q+R\left[f_{-1 / 2}, q\right]$, if $\varepsilon=1$. We also know $\mathfrak{r}=U+\mathfrak{n}$ and $\mathfrak{g}=\mathfrak{g}^{\prime}+U+j U$. Note that $\left(\mathfrak{g}_{1 / 2}+\mathfrak{g}_{-1 / 2}\right)$ modulo $\left(\mathfrak{g}_{1 / 2}^{\prime}+j \mathfrak{g}_{1 / 2}^{\prime}\right)$ is at most two-dimensional.

In the following sections we will exclude the four possibilities for $f_{0}: \lambda=0,1, \varepsilon= \pm 1$.

3.28. Since $\mathrm{g} / \mathfrak{g}^{\prime}$ is of low dimension it is natural to consider some of the cases $\mathfrak{g}_{\lambda}=\mathfrak{g}_{\lambda}^{\prime}$.

Lemma. The case $\mathfrak{g}_{1 / 2}=\mathfrak{g}_{\lambda}^{\prime}$ does not occur.

Proof. Suppose $\mathfrak{g}_{1 / 2}=\mathfrak{g}_{1 / 2}^{\prime}$. Then $\mathfrak{g}_{1 / 2}=\mathfrak{u}_{1 / 2}+\mathfrak{w}_{1 / 2}$, where $\mathfrak{u}_{1 / 2}$ and $\mathfrak{w}_{1 / 2}$ are defined as in 3.7. Recall $\mathfrak{u}_{1 / 2}=j \mathfrak{n}_{-1 / 2}=\left[e, \mathfrak{n}_{-1 / 2}\right] \subset \mathfrak{n}$, whence - in $\mathfrak{g}^{\prime}$ $\left[\mathfrak{w}_{1 / 2}, \mathfrak{u}_{1 / 2}\right]=0$. Under our assumptions we also know that $\mathfrak{H}_{1 / 2}$ is the bilinear kernel of $\rho$ restricted to $g_{1 / 2}$. The closedness condition for $\rho$ implies that $\mathfrak{w}_{0}+t_{0}$ leaves $\mathfrak{u}_{1 / 2}$ invariant. From this and the integrability condition it follows that $\mathfrak{w}_{0}+t_{0}$ acts symplectically (in the sense of $[7 ; \S 6]$ ) on $\mathfrak{g}_{1 / 2}^{\prime} / \mathfrak{u}_{1 / 2}$. Therefore $\mathfrak{w}_{0}$ has only the (real) eigenvalue 0 and $j q$ has only the (real) eigenvalues $0, \pm 1 / 2$. Hence, the eigenvalues of $f_{0}$ on $g_{1 / 2}$ are $\lambda / 2$ and $\lambda / 2 \pm \varepsilon / 2$. It is easy to see that for $\lambda=0,1$ and $\varepsilon= \pm 1$ the eigenvalue 2 does not occur. But then $\mathfrak{h}_{1 / 2}=0$, a contradiction.

\subsection{9.}

LEMma. The case $f_{0}=\lambda j e+j q+w_{0}$ does not occur.

Proof. By 3.28 we can assume $\mathfrak{g}_{1 / 2} \neq \mathfrak{g}_{1 / 2}^{\prime}$. Since, by 3.27, $\left(\mathfrak{g}_{1 / 2}+\mathfrak{g}_{-1 / 2}\right)$ modulo $\left(\mathfrak{g}_{1 / 2}^{\prime}+\mathfrak{g}_{-1 / 2}^{\prime}\right)$ is at most two-dimensional, we know $\mathfrak{g}_{-1 / 2}=\mathfrak{g}_{-1 / 2}^{\prime}+$ $\boldsymbol{R} v_{-1 / 2}$. In particular $f_{-1 / 2}=j u_{1 / 2}+b v_{-1 / 2}$ for some $u_{1 / 2} \in \mathfrak{u}_{1 / 2} \subset \mathfrak{n}, \boldsymbol{b} \in \boldsymbol{R}$. This implies $\rho\left(j u_{1 / 2}, u_{1 / 2}\right)=\rho\left(f_{-1 / 2}-b v_{-1 / 2}, u_{1 / 2}\right)$. We want to show that this expression vanishes. Then $u_{1 / 2}=0$ and $f_{-1 / 2} \in \boldsymbol{R \mathfrak { v }}_{-1 / 2} \subset \mathfrak{x} \subset \operatorname{nil}(\mathfrak{g})$, a contradiction. To see that $\rho\left(j u_{1 / 2}, u_{1 / 2}\right)$ vanishes we note that $f_{0}$ has the eigenvalue -2 on $f_{-1 / 2}$ and -1 on $\mathfrak{v}_{-1 / 2}$. In the situation under consideration $f_{0}$ can only have the eigenvalues $2,-1,0$ on $g_{1 / 2}$. We note that $(\nu+\mu) \rho\left(x_{-1 / 2}^{(\nu)}, n_{1 / 2}^{(\mu)}\right)=\rho\left(\left[f_{0}, x_{-1 / 2}^{(\nu)}\right], n_{1 / 2}^{(\mu)}\right)+\rho\left(x_{-1 / 2}^{(\nu)},\left[f_{0}, n_{1 / 2}^{(\mu)}\right]\right)=\rho\left(f_{0},\left[x_{-1 / 2}^{(\nu)}, n_{1 / 2}^{(\mu)}\right]\right)$ holds. If $n_{1 / 2}^{(\mu)} \in \mathfrak{n}$, then $\left[x_{-1 / 2}^{(\nu)}, n_{1 / 2}^{(\mu)}\right] \in \mathfrak{n}_{0}^{(\nu+\mu)}$. This expression vanishes if $\nu+\mu \neq 0$ as shown in 3.18. Therefore $\rho\left(\mathfrak{g}_{-1 / 2}^{(\nu)}, \mathfrak{n}_{1 / 2}^{(\mu)}\right)=0$ if $\nu+\mu \neq 0$. This applies in particular to $\nu=-2,-1$ and $\mu=0,-1$. To finish the proof of this lemma it suffices to show $\mathfrak{n}_{1 / 2}^{(2)}=0$. But if $\mathfrak{n}_{1 / 2}^{(2)} \neq 0$, then also $0 \neq\left[f_{-1 / 2},\left[f_{-1 / 2}, \mathfrak{n}_{1 / 2}^{(2)}\right]\right] \subset \mathfrak{n}_{-1 / 2}^{(-2)}$. To see that this is impossible we consider 
the space $\mathfrak{b}=\mathfrak{n}_{-1 / 2}=j \mathfrak{n}_{-1 / 2}$. Since $j \mathfrak{n}_{-1 / 2}=\left[e, \mathfrak{n}_{-1 / 2}\right]$ we see that $\mathfrak{b}$ is invariant under $\mathfrak{w}_{0}+t_{0}$. It is easy to see that the representation of $\mathfrak{w}_{0}+t_{0}$ on $\mathfrak{v}$ is symplectic. Therefore $j q$ has only the (real) eigenvalues $0, \pm 1 / 2$ and $w_{0}$ only the (real) eigenvalue 0 on $\mathfrak{b}$. Thus $f_{0}$ cannot have the eigenvalue -2 on $\mathfrak{v}$. This contradiction finishes the proof of the lemma.

3.30. In this section we finish the proof of "Case 3 " by showing

Lemma. The case $f_{0}=\lambda j e-j q+w_{0}$ does not occur.

Proof. By 3.28 we can assume $\mathfrak{g}_{1 / 2} \neq \mathfrak{g}_{1 / 2}^{\prime}$. We also know $U=R q+R v_{1 / 2}$ and $\mathfrak{g}=\mathfrak{g}^{\prime}+U+j U$. In particular $\mathfrak{g}_{-1 / 2}=\mathfrak{g}_{-1 / 2}^{\prime}+\boldsymbol{R}\left(j v_{1 / 2}\right)_{-1 / 2}$. Splitting $v_{1 / 2}=u_{1 / 2}+w_{1 / 2}$ where $u_{1 / 2} \in \mathfrak{u}_{1 / 2} \subset \mathfrak{n}$ and $w_{1 / 2} \in \mathfrak{w}_{1 / 2}$ (see 3.7) we see $j v_{1 / 2}=$ $j u_{1 / 2}+j w_{1 / 2}$, hence $\left(j v_{1 / 2}\right)_{-1 / 2}=j u_{1 / 2} \in \mathfrak{g}^{\prime}$. Therefore $\mathfrak{g}_{-1 / 2}=\mathfrak{g}_{-1 / 2}^{\prime}$. But then $j \mathfrak{g}_{-1 / 2}=\left[e, \mathfrak{g}_{-1 / 2}\right]$ and $\mathfrak{v}=\mathfrak{g}_{-1 / 2}+j \mathfrak{g}_{-1 / 2}$ is left invariant by $\mathfrak{w}_{0}+\mathfrak{t}_{0}$. From this it follows that $j q$ has only the (real) eigenvalues $0, \pm 1 / 2$ and $w_{0}$ has only the (real) eigenvalue 0 on $\mathfrak{v}$. Therefore $f_{0}$ does not have the eigenvalue -2 on $\mathfrak{g}_{-1 / 2}$ and the lemma is proven.

APPENDIX. We want to prove the following general result.

LEMMA. Let $\mathfrak{q}$ be an algebraic Lie algebra of endomorphisms of some vector space $V$ and $\mathfrak{b} \subset \mathfrak{q}$ an abelian sabspace such that every $b \in \mathfrak{b}$ is $a$ semisimple endomorphism of $V$.

Then there exists a maximal semisimple subalgebra $\mathfrak{h}$ of $\mathfrak{q}$ and an algebraic abelian subalgebra $\mathfrak{a} \subset \operatorname{rad}(\mathfrak{g})$ that consists of semisimple endomorphisms such that

a) $[\mathfrak{h}, \mathfrak{a}]=0$,

b) $\mathfrak{b} \subset \mathfrak{h}+\mathfrak{a}$.

Proof. We prove the assertion by induction on $m=\operatorname{dim} \mathfrak{b}$. If $m=1$ it suffices to consider some $0 \neq q \in \mathfrak{b}$. From [3, chap. VI, $\S 4$, Proposition 18] we know that there exists a Cartan subalgebra $\mathfrak{c}$ of $\mathfrak{q}$ containing $q$. Hence by loc. cit. Proposition 20 there exists a maximal semisimple subalgebra $\mathfrak{h}^{\prime}$ of $\mathfrak{q}$ such that $c_{h}^{\prime}=\mathfrak{h}^{\prime} \cap \mathfrak{c}$ is a Cartan subalgebra of $\mathfrak{h}^{\prime}$ and $\mathfrak{c}=\mathfrak{c}_{h}^{\prime}+(\mathfrak{c} \cap \operatorname{rad}(\mathfrak{q}))$. We note that $\mathfrak{c}_{h}^{\prime}$ consists of semisimple endomorphisms of $V$. From [3, chap. V, §4, Proposition 5] we derive that we can write $\operatorname{rad}(\mathfrak{q})=\mathfrak{a}^{\prime}+\mathfrak{n}$ where $\mathfrak{a}^{\prime}$ is abelian, algebraic and commutes with $\mathfrak{h}^{\prime}$ and where $\mathfrak{n} \subset \operatorname{rad}(\mathfrak{q})$ is the greatest ideal of $\mathfrak{q}$ consisting of nilpotent endomorphisms. Then $q=h+q+n$ and $h+q$ is semisimple. We write 
$n=n_{0}+n_{1}$ where $n_{0}$ is in the kernel of $H=\operatorname{ad}(h+a)$ and $n_{1}$ is in the sum $\mathfrak{w}$ of the eigenspaces of $H$ for eigenvalues $\lambda \neq 0$. Clearly, $H$ is invertible on $\mathfrak{w}$. We denote by $\mathfrak{n}^{(k)}$ the space of $k$-fold commutators of elements from $\mathfrak{n}, \mathfrak{n}^{(1)}=\mathfrak{n}$. Then $\mathfrak{n}^{(k)}$ is left invariant by $H$. Since $\mathfrak{n}^{(k)} \supset$ $\mathfrak{n}^{(k+1)}$ there exists some $H$-invariant complement $\mathfrak{u}^{(k)}$ of $\mathfrak{n}^{(k+1)}$ in $\mathfrak{n}^{(k)}$. We note that for every eigenspace $\mathfrak{b}_{\lambda}$ of $H$ we have $\mathfrak{b}_{\lambda} \cap \mathfrak{n}^{(k)}=\mathfrak{b}_{\lambda} \cap \mathfrak{u}^{(k)}+$ $\mathfrak{b}_{\lambda} \cap \mathfrak{n}^{(k+1)}$. We write $n_{0}=u_{0}^{(1)}+n_{0}^{(2)}$ and $n_{1}=u_{1}^{(1)}+n_{1}^{(2)}$; then $u^{(1)}, n_{0}^{(2)}$ both are in the kernel of $H$ and $H$ is invertible on $\mathfrak{u}^{(1)} \cap \mathfrak{w}$. Let $A_{1}=$ $\exp \operatorname{ad}\left(H^{-1} u_{1}^{(1)}\right)$. Then $A_{1}(h+a+n)=h+a+n-\left[h+a+n, H^{-1} u_{1}^{(1)}\right] \bmod \mathfrak{n}^{(2)}$ $=h+a+u_{0}^{(1)}+u_{1}^{(1)}-u^{(1)} \bmod \mathfrak{n}^{(2)}=h+a+u_{0}^{(1)} \bmod \mathfrak{n}^{(2)}$. We iterate this procedure and assume that we have found already inner automorphisms $A_{1}, \cdots, A_{r-1}$ so that $A_{r-1}, \cdots, A_{1}(h+a+n)=h+a+u_{0}^{(1)}+u_{0}^{(2)}+\cdots$ $+u_{0}^{(r-1)}+n^{(r)}$ for some $n^{(r)} \in \mathfrak{n}^{(r)}$. We write $n^{(r)}=n_{0}^{(r)}+n_{1}^{(r)}$ and $n_{j}^{(r)}=$ $u_{j}^{(r)}+n_{j}^{(r+1)}, j=0,1$, with $n_{0}^{(r)} \in \operatorname{ker} H$ and $n_{j}^{(r)} \in \mathfrak{w}$. Set $A_{r}=\exp \operatorname{ad}\left(H^{-1} u_{1}^{(r)}\right)$. Then $A_{r}, \cdots, A_{1}(h+a+n)=A_{r}\left(h+a+u_{0}^{(1)}+\cdots+u_{0}^{(r-1)}+n^{(r)}\right)=h+$ $a+u_{0}^{(1)}+\cdots+u_{0}^{(r)}+u_{1}^{(r)}-\left[h+a+u_{0}^{(1)}+\cdots, H^{-1} u_{1}^{(r)}\right] \bmod \mathfrak{n}^{(r+1)}=h+$ $a+u_{0}^{(1)}+\cdots+u_{0}^{(r)} \bmod \mathfrak{n}^{(r+1)}$. Hence we find an inner automorphism of $\mathfrak{q}$ such that $W(h+a+n)=h+a+x$ where $[h+a, x]=0$. But $W(h+a+n)$ and $h+a$ are semisimple endomorphisms and $x$ is nilpotent. Therefore $x=0$. We set $\mathfrak{h}=W^{-1} \mathfrak{h}^{\prime}$ and $\mathfrak{a}=W^{-1} \mathfrak{a}^{\prime}$. Then $q=W^{-1} h+W^{-1} a \in \mathfrak{h}+\mathfrak{a}$ and the assertion follows.

Assume now $\operatorname{dim} \mathfrak{b}=m$ and the assertion holds for dimensions less than $m$. We write $\mathfrak{b}=\mathfrak{b}^{\prime} \oplus \boldsymbol{R} q$ and apply the induction hypothesis to $\mathfrak{b}^{\prime}$. The corresponding subalgebras will be denoted by $\mathfrak{h}^{\prime}$ and $\mathfrak{a}^{\prime}$. Hence $b^{\prime}=h^{\prime}+a^{\prime}$, where $h^{\prime} \in \mathfrak{h}^{\prime}, a^{\prime} \in \mathfrak{a}^{\prime}$, for all $b^{\prime} \in \mathfrak{b}^{\prime}$ and $q=h+a+n$ where $\mathfrak{n}$ is as above. Since $\left[b^{\prime}, q\right]=0$ we have

$$
\begin{aligned}
& {\left[b^{\prime}, h+a\right]=0,} \\
& {\left[b^{\prime}, n\right]=0 .}
\end{aligned}
$$

Now we repeat the proof above and note that in every step the inner automorphisms $A_{i}$ fix $\mathfrak{b}^{\prime}$. From this the assertion follows.

Added in proof. The following paper builds on the present article: J. Dorfmeister, K. Nakajima. The fundamental conjecture for homogeneous Kähler manifolds, Acta Math., 161 (1988), 23-70. 


\section{LITERATURE}

[1] R. Azencott, E. N. Wilson, Homogeneous manifolds with negative curvature I, Trans. Amer. Math. Soc., 215 (1976), 373-362.

[2] N. Bourbaki, Groupes et algèbres de Lie, chap. I, Actualités Scientifiques et industrielles 1285, Hermann, Paris 1971.

[ 3 ] C. Chevalley, Théorie des groupes de Lie, Hermann, Paris, 1968.

[ 4 ] J. E. D'Atri, J. Dorfmeister, Zhao Yan da, The isotropy representation for homogeneous Siegel domains, Pacific J. Math., 120 (1985), 295-326.

[5] J. Dorfmeister, Homogeneous Kähler manifolds admitting a transitive solvable group of automorphisms, Ann. scient. Èc. Norm. Sup., 18 (1985), 143-180.

[6] — The Radical Conjecture for Homogeneous Kähler Manifolds, in CMS Conference Proceedings, Vol. 5 (1986), 189-208.

[ 7 ] S. G. Gindikin, I. I. Piatetskii-Shapiro, E. B. Vinberg, Homogeneous Kähler manifolds, in "Geometry of homogeneous bounded domains", CrME, Italy, 1967.

[8] - On the classification and canonical realization of homogeneous bounded domains, Trans. Moscow Math. Soc., 12 (1963), 404-437.

[ 9 ] K. Nakajima, Homogeneous hyperbolic manifolds and homogeneous Siegel domains, J. Math. Kyoto Univ., 25 (1985), 269-291.

[10] — On J-algebras and homogeneous Kähler manifolds, Hokkaido Math. J., 15 (1986), 1-20.

[11] O. Kühn, A. Rosendahl, Wedderburnzerlegung für Jordan-Paare, Manuscripta math., 24 (1978), 403-435.

[12] E. Neher, Cartan-Involutionen von halbeinfachen reellen Jordan-Tripelsystemen, Math. Z., 169 (1979), 271-292.

[13] —-, Klassifikation der einfachen reellen speziellen Jordan-Tripelsysteme, Manuscripta math., 31 (1980), 197-215.

[14] - Jordan triple systems with completely reducible derivation or structure algebras, Pacific J. Math., 113 (1984), 137-164.

[15] — Jordan triple systems by the Grid approach, Lecture Notes in Math., 1280, Springer Verlag.

[16] A. L. Oniscik, Inclusion relations among transitive compact transformation groups, Amer. Math. Soc. Translations, Ser. 2, 50 (1966), 5-58.

[17] E. B. Vinberg, The structure of the group of automorphisms of a homogeneous convex cone, Trans. Moscow Math. Soc., 13 (1965), 63-93.

Department of Mathematics

University of Kansas

Lawrence, Kansas 66045

U.S.A. 